\title{
Construction and Analysis of Lattice Boltzmann Methods Applied to a 1D Convection-Diffusion Equation
}

\author{
Stéphane Dellacherie \\ Commissariat l'Énergie Atomique et aux Énergies Alternatives \\ CEA, DEN, DM2S, STMF \\ F-91191 Gif-sur-Yvette \\ France
}

\begin{abstract}
We construct and we analyze two LBM schemes build on the D1Q2 lattice to solve the 1D (linear) convectiondiffusion equation. We obtain these LBM schemes by showing that the 1D convection-diffusion equation is the fluid limit of a discrete velocity kinetic system. Then, we show in the periodic case that these LBM schemes are equivalent to a finite difference type scheme named LFCCDF scheme. This allows us, firstly, to prove the convergence in $L^{\infty}$ of these schemes, and to obtain discrete maximum principles for any time step in the case of the 1D diffusion equation with different boundary conditions. Secondly, this allows us to obtain most of these results for the Du Fort-Frankel scheme for a particular choice of the first iterate. We also underline that these LBM schemes can be applied to the (linear) advection equation and we obtain a stability result in $L^{\infty}$ under a classical CFL condition. Moreover, by proposing a probabilistic interpretation of these LBM schemes, we also obtain Monte-Carlo algorithms which approach the 1D (linear) diffusion equation. At last, we present numerical applications justifying these results.
\end{abstract}

Key words:

Convection-diffusion equation, Lattice Boltzmann method, D1Q2 lattice, Chapman-Enskog expansion, Hilbert expansion, LFCCDF scheme, Du Fort-Frankel scheme, stability and convergence in $\mathrm{L}^{\infty}$, discrete maximum principle, Monte-Carlo algorithm.

\section{Introduction}

Lattice Boltzmann Methods (LBM) come from an attempt to simulate the incompressible Navier-Stokes system with cellular automata $[1,2]$ before being seen as deterministic schemes based on the resolution of discrete velocity kinetic systems [3]. LBM schemes are now often used to solve many type of Partial Differential Equations (PDEs) system. Among them, we find the heat equation with or without phase change $[4,5,6,7]$, the heat equation with radiative source term [8], the hyperbolic heat equation (also named telegraph equation) with or without radiative source term [9], the Richard equation for porous media [10], the advection-diffusion equation [11, 12, 13, 14, 15, 16, 17], the advection equation [18], the incompressible Navier-Stokes system [19] eventually applied in porous media with heat and mass transfer [20] or in a diphasic situation [21, 22] or with a free-surface [23], the Bingham model for viscoplastic flows [24]. Among the reasons which justify the use of LBM schemes, we can cite its algorithmic simplicity, its time

Email address: stephane.dellacherie@cea.fr (Stéphane Dellacherie)

Preprint submitted to Acta Applicandae Mathematicae

September 18, 2013 
explicit nature, its scalability when the algorithm is parallelized. We can also cite the fact that all LBM schemes are formulated in a common way. More precisely, when $W(t, x)$ is the solution of a PDEs system $\partial_{t} W+\mathcal{L}(W)=0$ where $t \geq 0$ is the time, $x \in \Omega \subset \mathbb{R}^{d}$ is the spacial variable $(d \in\{1,2,3\})$ and $\mathcal{L}(\cdot)$ is a spatial differential operator, the approximation $W_{i}^{n+1}$ of $W\left(t^{n+1}, x_{i}\right)$ is obtained through a formula of the type

$$
W_{i}^{n+1}=\sum_{q=1}^{q_{\max }} \varphi\left(v_{q}\right) f_{q, i}^{n+1}
$$

where $\varphi(\cdot)$ is a given function which depends on the PDEs system, where $v_{q} \in \mathbb{R}^{d}$ belongs to a discrete and finite set $\left\{v_{q}\right\}_{1 \leq q \leq q_{\max }}$ and where $f_{q, i}^{n+1} \in \mathbb{R}$ is deduced from a scheme of the type

$$
f_{q, i}^{n+1}=L_{q, i}^{n} \cdot \mathbf{f}_{i}^{n} .
$$

Above, $L_{q, i}^{n} \in \mathbb{R}^{1 \times M}$ depends on $\left\{W_{j_{i}}^{n}\right\}_{j_{i}}$ where $j_{i}$ belongs to a stencil $\left\{j_{i}\right\}$ centered on $i\left(e . g .\left\{j_{i}\right\}=\{i-1, i, i+\right.$ $1\})$ and $\mathbf{f}_{i}^{n}:=\left[\left(f_{1, j_{i}}\right)_{j_{i}}, \ldots,\left(f_{q_{\max }, j_{i}}\right)_{j_{i}}\right] \in \mathbb{R}^{M \times 1}$. Here, $M \in \mathbb{N}$ is equal to $q_{\max } \times$ card $\left\{j_{i}\right\}$ (let us note that card $\left\{j_{i}\right\}$ - and, thus, $M$ - may depend on $i$ when the boundary conditions on $\partial \Omega$ are not periodic). In the LBM realm, $f_{q, i}^{n}$ is sometimes interpreted as a density of particles having the velocity $v_{q}$ at the time $t^{n}$ and at the node $x_{i}$ of the spatial lattice.

Although the LBM schemes have proven their efficiency both in academical and industrial situations (see for example the numerical applications in [23]), the numerical analysis of these schemes is not well developed. Moreover, this numerical approach suffers sometimes of a lack of precision in the way to introduce it. Nevertheless, there exists recent publications which give accurate informations on the LBM approach from a mathematical point of view. Among them, we find in $[25,26]$ a justification of LBM schemes applied to the 1D linear advection equation and to a 1D Navier-Stokes type model (which is a p-system with diffusive term). In [27, 28], a Taylor expansion is applied to the LBM scheme to justify it in the case of the 1D convection-diffusion equation and in the case of the 1D wave equation with diffusive term. In [29], a Chapman-Enskog expansion is applied to a LBM scheme in the case of the incompressible Navier-Stokes system. In [30, 31], a convergence result in $L^{2}$ is proposed for LBM schemes in the case of the incompressible Navier-Stokes system under some assumptions and a stability result in $L^{2}$ is obtained by linearizing the LBM schemes.

In this paper, we construct, we justify and we analyze two LBM schemes build on the D1Q2 lattice to solve the $1 \mathrm{D}$ convection-diffusion equation

$$
\left\{\begin{array}{l}
\partial_{t} \rho+\partial_{x}(u \rho)=v \partial_{x x}^{2} \rho, \\
\rho(0, x)=\rho^{0}(x)
\end{array}\right.
$$

where $t \geq 0$ and $x \in \Omega \subset \mathbb{R}$, where $v>0$ is the (constant) diffusion coefficient, $u(x)$ and $\rho^{0}(x)$ being given functions. In the pure diffusive case (i.e. $u=0$ ), one of these LBM schemes is classical and can be found in $[4,6,7,32]$ (the second one seems to be less classical). As in [29], these LBM schemes are obtained by discretizing a discrete velocity kinetic system whose the fluid limit is (1), this fluid limit being formally obtained with a Chapman-Enskog expansion and with a Hilbert expansion. Then, we prove convergence results in $L^{\infty}$ and discrete maximum principles when $u=0$ satisfied by the proposed LBM schemes with periodic, Neumann or Dirichlet boundary conditions. We also obtained two LBM schemes for the pure 
advection equation (i.e. $u$ is a constant and $v=0$ ) in the periodic case which converge in $L^{\infty}$ (under a classical CFL condition). Let us note that the $L^{2}$-stability of one of them is studied in [18] with a von Neumann stability analysis.

We underline that these convergence results are obtained by using in particular an equivalence result between the proposed LBM schemes and a finite difference type scheme named LFCCDF scheme [33]. For the classical LBM scheme that we propose, this equivalence was cited by Ancona [32] in the pure diffusive case with periodic boundary conditions, the LFCCDF scheme being the Du Fort-Frankel scheme [35] in that case. In the work by Suga [5] and by Ginzburg et al. [15, 16, 34], the possible existence of links between LBM schemes (built on lattices different than the D1Q2 lattice) and finite difference type schemes is also studied.

We also underline that our results in $L^{\infty}$ are stronger than any results in $L^{2}$, and that they are obtained by using convexity properties of the proposed LBM schemes and, thus, without using any discrete Fourier transform (up to our knowledge, $L^{2}$-stability results are obtained with von Neumann analysis in the LBM realm: see for example $[5,11,12,13,15,16,17,18])$. In particular, our approach allows us to study the stability with non-periodic boundary conditions and to obtain discrete maximum principles, which is not possible with a von Neumann analysis. This approach is possible because of the simplicity of the D1Q2 lattice. Nevertheless, it is not obvious that our approach would be possible for other lattices such as the D1Q3 lattice studied (among other lattices) in [5, 13, 15, 16, 17].

At last, we propose Monte-Carlo algorithms for the diffusion equation with periodic boundary conditions. These Monte-Carlo algorithms come from a probabilistic interpretation of the proposed LBM schemes and have links with the Monte-Carlo algorithm proposed in [41] for the telegraph equation. Some basic properties of these Monte-Carlo algorithms are proved (among them, we recover the discrete maximum principles obtained with the LBM schemes in the periodic case). Like for the finite difference schemes theory, we hope that this probabilistic approach will be a tool to analyze the LBM schemes.

The end of the paper is devoted to numerical simulations. These numerical results justify the discrete maximum principles obtained with the proposed LBM schemes (with periodic or non-periodic boundary conditions) and with the Monte-Carlo algorithms (with periodic boundary conditions). They also show the convergence of these algorithms and their robustness.

The outline of this paper is the following. In Section 2, we introduce the discrete velocity kinetic system and its fluid limit. In Section 3, we contruct the LBM schemes. In Section 4, we obtain the equivalence of these LBM schemes with the LFCCDF scheme (which is the Du Fort-Frankel scheme [35] when $u=0$ ). In Section 5, we obtain stability and converence results in $L^{\infty}$. In Section 6, we prove discrete maximum principles in the case of the diffusion equation. In Section 7, we present some limitations of the LBM schemes. In Section 8, we extend the proposed LBM schemes to the convection equation. In Section 9, a probabilistic interpretation of the LBM schemes is proposed in the case of the diffusion equation with periodic boundary conditions. In Section 10, we present some numerical results. At last, we conclude the paper in Section 11. 


\section{Fluid limit of a discrete velocity kinetic system}

Let us define the function

$$
M_{q}^{\varepsilon}(t, x):=\frac{\rho(t, x)}{2}\left[1+\frac{u(x)}{v_{q}^{\varepsilon}}\right]
$$

where $\rho(t, x) \in \mathbb{R}_{*}^{+}$and $u(x) \in \mathbb{R}$. The parameters $t \in \mathbb{R}^{+}$and $x \in \Omega:=\left[x_{\min }, x_{\max }\right](\Omega \subset \mathbb{R})$ are the time and space variables. The set $\left\{v_{q}^{\varepsilon}\right\}_{q \in\{1,2\}}$ is a discrete and finite set of velocities defined by

$$
v_{q}^{\varepsilon}:=(-1)^{q} c^{\varepsilon} \quad \text { with } \quad q \in\{1,2\}
$$

where $c^{\varepsilon} \in \mathbb{R}^{+}$depends on a parameter $\varepsilon \in \mathbb{R}^{+}$and will be defined later. In the LBM realm, $\left\{v_{q}^{\varepsilon}\right\}_{q \in\{1,2\}}$ is named D1Q2. The function $M_{q}^{\varepsilon}$ verifies

$$
\sum_{q \in\{1,2\}}\left(\begin{array}{c}
1 \\
v_{q}^{\varepsilon}
\end{array}\right) M_{q}^{\varepsilon}=\left(\begin{array}{c}
\rho \\
\rho u
\end{array}\right) .
$$

To solve the convection-diffusion equation (1), we will propose two schemes deduced from a numerical integration of the discrete velocity kinetic system

$$
\left\{\begin{array}{l}
\partial_{t} f_{1}^{\varepsilon}+v_{1}^{\varepsilon} \partial_{x} f_{1}^{\varepsilon}=\frac{1}{\varepsilon}\left(M_{1}^{\varepsilon}-f_{1}^{\varepsilon}\right) \\
\partial_{t} f_{2}^{\varepsilon}+v_{2}^{\varepsilon} \partial_{x} f_{2}^{\varepsilon}=\frac{1}{\varepsilon}\left(M_{2}^{\varepsilon}-f_{2}^{\varepsilon}\right),
\end{array}\right.
$$

and by previously noting that the fluid limit of (5) is (1) for a particular choice of $v_{q}^{\varepsilon}$ (see Proposition 2.1 below). These schemes will belong to the family of LBM schemes and will be explicited in $\$ 3$. In $\S 9$, we will show that the probabilistic interpretation of these LBM schemes are Monte-Carlo schemes which are already known. System (5) may be considered as a kinetic system whose the kinetic velocities belong to the discrete and finite set $\left\{v_{q}^{\varepsilon}\right\}_{q \in\{1,2\}}$ and whose the collision operator is a BGK-type collision operator where the classical maxwellian is replaced by $M_{q}^{\varepsilon}$ defined with (2). Thus, $M_{q}^{\varepsilon}$ will be named maxwellian in the sequel, $\rho(x)$ and $u(x)$ being the macroscopic density and velocity associated to $M_{q}^{\varepsilon}$. A similar approach is proposed in [29] in the case of the incompressible Navier-Stokes system. Nevertheless, the integration of (5) is obtained with a second order integration formula instead of a third order integration formula, which obliges the authors in [29] to correct the diffusion coefficient $v$ to obtain a consistent LBM scheme: this point is a direct consequence of the stiffness of (5) when $\varepsilon \ll 1$, and is clarified in $\S 3.4$.

At last, let us underline that most of the variables above depend on the collision time $\varepsilon$. Thus, to clearly underline this dependency, we use the superscript $\varepsilon$ in the notation. Nevertheless, we will simplify the notations after this section by omiting the parameter $\varepsilon$.

\subsection{The convection-diffusion equation as a fluid limit}

The fluid limit is obtained for a particular choice of the kinetic velocity $c^{\varepsilon}$ in (3):

Proposition 2.1. Let us suppose that $\Omega$ is periodic and let $f_{q}^{\varepsilon}$ be solution of the discrete velocity kinetic system

$$
\forall q \in\{1,2\}: \quad\left\{\begin{array}{l}
\partial_{t} f_{q}^{\varepsilon}+v_{q}^{\varepsilon} \partial_{x} f_{q}^{\varepsilon}=\frac{1}{\varepsilon}\left(M_{q}^{\varepsilon}-f_{q}^{\varepsilon}\right), \\
f_{q}^{\varepsilon}(t=0, x)=f_{q}^{\varepsilon, 0}(x)
\end{array}\right.
$$


where $M_{q}^{\varepsilon}:=\frac{\rho^{\varepsilon}(t, x)}{2}\left[1+\frac{u(x)}{v_{q}^{\varepsilon}}\right]$ with $\rho^{\varepsilon}:=f_{1}^{\varepsilon}+f_{2}^{\varepsilon}$. Let us also suppose that

$$
\left.v_{q}^{\varepsilon}=(-1)^{q} \sqrt{\frac{v}{\varepsilon}} \quad \text { (i.e. } c^{\varepsilon}=\sqrt{\frac{v}{\varepsilon}}\right) .
$$

Then, by admitting that there exists $\left\{g_{k, q}^{\varepsilon}(t, x)\right\}_{k \geq 1}$ in such a way

$$
f_{q}^{\varepsilon}=M_{q}^{\varepsilon} \cdot\left(1+\sqrt{\varepsilon} g_{1, q}^{\varepsilon}+\varepsilon g_{2, q}^{\varepsilon}+\varepsilon^{3 / 2} g_{3, q}^{\varepsilon}\right)+O\left(\varepsilon^{2}\right)
$$

when $\varepsilon \ll 1, \rho^{\varepsilon}$ is solution of

$$
\left\{\begin{array}{l}
\partial_{t} \rho^{\varepsilon}+\partial_{x}\left(u \rho^{\varepsilon}\right)=v \partial_{x x}^{2} \rho^{\varepsilon}+O(\varepsilon) \\
\rho^{\varepsilon}(t=0, x)=f_{1}^{\varepsilon, 0}(x)+f_{2}^{\varepsilon, 0}(x)
\end{array}\right.
$$

Moreover, we have

$f_{q}^{\varepsilon}(t, x)=M_{q}^{\varepsilon}+(-1)^{q+1} \frac{\sqrt{\varepsilon v}}{2} \partial_{x} \rho^{\varepsilon}+(-1)^{q} \varepsilon^{3 / 2}\left[\frac{u}{2 \sqrt{v}} \cdot\left(\partial_{x}\left(u \rho^{\varepsilon}\right)-v \partial_{x x}^{2} \rho^{\varepsilon}\right)-\frac{\sqrt{v}}{2} \partial_{x x}^{2}\left(u \rho^{\varepsilon}\right)+\frac{v^{3 / 2}}{2} \partial_{x x x}^{3} \rho^{\varepsilon}\right]+O\left(\varepsilon^{2}\right)$.

Proposition 2.1 means that the convection-diffusion equation (1) is the fluid limit of the discrete velocity kinetic system (6) when $v_{q}^{\varepsilon}$ is defined with (7). Let us note that $\varepsilon \ll 1$ means that $\varepsilon \ll t_{\text {fluid }}$ where $t_{\text {fluid }}=O(1)$ is the time scale of the fluid limit (1), $\varepsilon$ being the kinetic time scale ((1) and (6) are supposed to be dimensionless). Let us also note that the initial condition $f_{q}^{\varepsilon, 0}$ should be close to the maxwellian equilibrium $M_{q}^{\varepsilon}$ in such a way the ansatz (8) is valid at $t=0\left(\right.$ e.g. $\left.f_{q}^{\varepsilon, 0}=M_{q}^{\varepsilon}\right)$.

The proof of Proposition 2.1 is formal and is written in Annex A. It is based on a Chapman-Enskog expansion (Annex A.1) or on a Hilbert expansion (Annex A.2). The fact that the discrete velocity kinetic system (6) is such that the kinetic velocity $v_{q}^{\varepsilon}$ depends on the collision time $\varepsilon$ is not classical, and obliges us to perform carefully these expansions. We underline that if the boundary conditions were not periodic, this would introduce difficulties which are note studied in this paper (because of the possible existence of a Knudsen layer in the vicinity of $\partial \Omega$ in that case). Nevertheless, we will propose Neumann and Dirichlet boundary conditions in the sequel although they will not be deduced from any asymptotic expansion applied to (6).

\subsection{The diffusion equation as a fluid limit}

Of course, we deduce from Proposition 2.1 the following corollary:

Corollary 2.1. Let us suppose that $\Omega$ is periodic and let $f_{q}^{\varepsilon}$ be solution of the discrete velocity kinetic system

$$
\left\{\begin{array}{l}
\partial_{t} f_{1}^{\varepsilon}-\sqrt{\frac{v}{\varepsilon}} \partial_{x} f_{1}^{\varepsilon}=\frac{1}{2 \varepsilon}\left(f_{2}^{\varepsilon}-f_{1}^{\varepsilon}\right) \\
\partial_{t} f_{2}^{\varepsilon}+\sqrt{\frac{v}{\varepsilon}} \partial_{x} f_{2}^{\varepsilon}=\frac{1}{2 \varepsilon}\left(f_{1}^{\varepsilon}-f_{2}^{\varepsilon}\right) \\
f_{1}^{\varepsilon}(t=0, x)=f_{1}^{\varepsilon, 0}(x) \\
f_{2}^{\varepsilon}(t=0, x)=f_{5}^{\varepsilon, 0}(x)
\end{array}\right.
$$


Then, when $\varepsilon \ll 1, \rho^{\varepsilon}:=f_{1}^{\varepsilon}+f_{2}^{\varepsilon}$ is solution of

$$
\left\{\begin{array}{l}
\partial_{t} \rho^{\varepsilon}=v \partial_{x x}^{2} \rho^{\varepsilon}+O(\varepsilon) \\
\rho^{\varepsilon}(t=0, x)=f_{1}^{\varepsilon, 0}(x)+f_{2}^{\varepsilon, 0}(x)
\end{array}\right.
$$

Moreover, we have

$$
f_{q}^{\varepsilon}(t, x)=\frac{\rho^{\varepsilon}}{2}\left[1+(-1)^{q} \sqrt{\varepsilon v}\left(\varepsilon v \frac{\partial_{x x x}^{3} \rho^{\varepsilon}}{\rho^{\varepsilon}}-\frac{\partial_{x} \rho^{\varepsilon}}{\rho^{\varepsilon}}\right)\right]+O\left(\varepsilon^{2}\right) .
$$

Let us note that it is proven in [36] that the fluid limit of

$$
\left\{\begin{array}{l}
\partial_{t} \rho+\partial_{x} w=0 \\
\partial_{t} w+\partial_{x} p(\rho)=-\frac{w}{\varepsilon}
\end{array}\right.
$$

is given by

$$
\partial_{t} \rho=\varepsilon \partial_{x x}^{2} p(\rho)
$$

$p(\rho)$ being a given function which does not depend on $\varepsilon$. On the other side, we can note that when $p(\rho)=c^{2} \rho$ where $c$ is a constant, by defining $f_{1}$ and $f_{2}$ in such a way $\rho=f_{1}+f_{2}$ and $w=c\left(f_{2}-f_{1}\right)$, (14) is equivalent to

$$
\left\{\begin{array}{l}
\partial_{t} f_{1}-c \partial_{x} f_{1}=\frac{1}{2 \varepsilon}\left(f_{2}-f_{1}\right) \\
\partial_{t} f_{2}+c \partial_{x} f_{2}=\frac{1}{2 \varepsilon}\left(f_{1}-f_{2}\right)
\end{array}\right.
$$

whose the fluid limit is given by

$$
\partial_{t} \rho=\varepsilon c^{2} \partial_{x x}^{2} \rho .
$$

The discrete velocity kinetic system (15) is similar to (11). Nevertheless, the kinetic velocity $c$ in (15) is a constant and, thus, does not depend on $\varepsilon$ which is not the case of the kinetic velocity $c^{\varepsilon}:=\sqrt{\frac{v}{\varepsilon}}$ in (11). As a consequence, the fluid limits (12) and (16) are different.

\section{Construction of two LBM schemes}

To simplify the notations, we now omit $\varepsilon$ in $f_{q}^{\varepsilon}$ and $M_{q}^{\varepsilon}$. Let us now define a 1D mesh $\left\{x_{i}\right\}_{i \in\{1, \ldots, N\}}$ of the domain $\Omega:=\left[x_{\min }, x_{\max }\right]$ whose the mesh size $\Delta x$ is constant, and let us define an uniform time step $\Delta t=t^{n+1}-t^{n}$ such that

$$
\Delta t:=C_{d} \frac{\Delta x^{2}}{v} \quad \text { with } \quad C_{d}=O(1) .
$$

This definition is justified by the fact that we want to discretize with an explicit scheme a convectiondiffusion equation. Proposition 2.1 encourages us to discretize

$$
\forall q \in\{1,2\}: \quad\left\{\begin{array}{l}
\partial_{t} f_{q}+(-1)^{q} \sqrt{\frac{\nu}{\varepsilon}} \partial_{x} f_{q}=\frac{1}{\varepsilon}\left(M_{q}-f_{q}\right), \\
f_{q}(t=0, x)=f_{q}^{0}(x)
\end{array}\right.
$$


instead of

$$
\left\{\begin{array}{l}
\partial_{t} \rho+\partial_{x}(u \rho)=v \partial_{x x}^{2} \rho, \\
\rho(t=0, x)=\rho^{0}(x) .
\end{array}\right.
$$

The difficulty is to find an explicit scheme applied to (18) which is enough accurate to capture the fluid limit (19) when $\Delta t=O\left(\Delta x^{2}\right) \ll 1$ and $\varepsilon \ll 1$. The solution will be to choose $\varepsilon=O(\Delta t)$, which implies that $\varepsilon$ will depend on $\Delta t$ : this dependency makes difficult the analysis of the LBM schemes.

\subsection{Integration of the kinetic system}

We have the following result whose the proof is based on an idea that we can find in $[37,25]$ :

Proposition 3.1. Let $c \in \mathbb{R}$ and $f_{q}(t, x)$ be solution of the kinetic system

$$
\forall q \in\{1,2\}: \quad \partial_{t} f_{q}+(-1)^{q} c \partial_{x} f_{q}=\frac{1}{\varepsilon}\left(M_{q}-f_{q}\right):=Q_{q}(f)(t, x)
$$

and let

$$
g_{q}(t, x):=f_{q}(t, x)-\frac{\Delta t}{2} Q_{q}(f)(t, x)
$$

Then, we have

$$
g_{1}+g_{2}=f_{1}+f_{2}
$$

and

$$
g_{q}\left[t+\Delta t, x+(-1)^{q} c \Delta t\right]=g_{q}(t, x)(1-\eta)+M_{q}(t, x) \eta+O\left(\frac{\Delta t^{3}}{\sqrt{\varepsilon}}\right) \quad \text { when } \quad \Delta t^{3} \ll \sqrt{\varepsilon}
$$

where

$$
\eta=\frac{1}{\frac{\varepsilon}{\Delta t}+\frac{1}{2}} .
$$

Since $g_{1}+g_{2}=f_{1}+f_{2}=: \rho$, we will propose LBM schemes by using the approximation (21): this point underlines also that the LBM scheme will be based on the intermediate variable $g_{q}$ instead of $f_{q}$. Nevertheless, it will be possible to deduce LBM schemes based on $f_{q}$ by applying the inverse transform of (20) to the LBM schemes based on $g_{q}$.

The proof of Proposition 3.1 is based on the following lemma:

Lemma 3.1. Let us define the BGK kernel

$$
Q(f)=\frac{1}{\varepsilon}\left(\mathcal{M}_{f}-f\right) \quad(\varepsilon \in \mathbb{R})
$$

where the distribution $f(v)$ is defined on a discrete or continuous velocity domain, $\mathcal{M}_{f}$ being the maxwellian distribution associated to $f$, and let us define the distribution

$$
g:=f-\widetilde{\varepsilon} Q(f) \quad(\widetilde{\varepsilon} \in \mathbb{R}) .
$$

Then, when $\widetilde{\varepsilon} \neq-\varepsilon$ :

$$
Q(f)=\frac{\varepsilon}{\varepsilon+\widetilde{\varepsilon}} Q(g)
$$


Let us underline that Lemma 3.1 is not restricted to a discrete velocity domain.

Proof of Proposition 3.1: The solution of the continuous EDP

$$
\partial_{t} f_{q}+(-1)^{q} c \partial_{x} f_{q}=\frac{1}{\varepsilon}\left(M_{q}-f_{q}\right)=: Q_{q}(f)(t, x)
$$

is given by

$$
f_{q}\left[t+\Delta t, x+(-1)^{q} c \Delta t\right]=f_{q}(t, x)+\int_{0}^{\Delta t} Q_{q}(f)\left[t+s, x+(-1)^{q} c s\right] d s .
$$

Since $f_{q}^{\varepsilon}=M_{q}^{\varepsilon}+O(\sqrt{\varepsilon})$ when $\varepsilon \ll 1$ (see (10)), we can write that

$$
Q_{q}^{\varepsilon}=O\left(\frac{1}{\sqrt{\varepsilon}}\right) \quad \text { when } \quad \varepsilon \ll 1 \text {. }
$$

This implies that

$$
\int_{0}^{\Delta t} Q_{q}(f)\left[t+s, x+(-1)^{q} c s\right] d s=O\left(\frac{\Delta t}{\sqrt{\varepsilon}}\right) \quad \text { when } \quad \varepsilon \ll 1 .
$$

Thus, the easiest numerical integration formula applied to (23) would give

$$
f_{q}\left[t+\Delta t, x+(-1)^{q} c \Delta t\right]=f_{q}(t, x)+\Delta t Q_{q}(f)(t, x)+O\left(\frac{\Delta t^{2}}{\sqrt{\varepsilon}}\right) .
$$

Nevertheless, the error $O\left(\Delta t^{2} / \sqrt{\varepsilon}\right)$ in (26) does not allow to obtain LBM schemes which are consistent with the convection-diffusion equation (19). In fact, the integration error has to be of the order of $\Delta t^{3} / \sqrt{\varepsilon}$ (or lower) for reasons that we justify in §3.4: this point is important in the LBM realm and explains the classical formula

$$
v=\Delta t\left(\frac{1}{\eta}-\frac{1}{2}\right) c_{s}^{2}
$$

met in many LBM schemes ( $\eta$ and $c_{s}$ are defined in \$3.2). Thus, instead of the second order integration formula (26), we use the third order integration formula

$$
f_{q}\left[t+\Delta t, x+(-1)^{q} c \Delta t\right]=f_{q}(t, x)+\frac{\Delta t}{2}\left[Q_{q}(f)(t, x)+Q_{q}(f)\left(t+\Delta t, x+(-1)^{q} c \Delta t\right)\right]+O\left(\frac{\Delta t^{3}}{\sqrt{\varepsilon}}\right) .
$$

Formula (27) is equivalent to $g_{q}\left[t+\Delta t, x+(-1)^{q} c \Delta t\right]=g_{q}(t, x)+\Delta t Q_{q}(f)(t, x)+O\left(\frac{\Delta t^{3}}{\sqrt{\varepsilon}}\right)$ with $g_{q}:=f_{q}-$ $\frac{\Delta t}{2} Q_{q}(f)$. Thus, by using Lemma 3.1 with $\widetilde{\varepsilon}=\Delta t / 2$, we obtain that (27) is equivalent to

$$
g_{q}\left[t+\Delta t, x+(-1)^{q} c \Delta t\right]=g_{q}(t, x)+\frac{\varepsilon}{\frac{\varepsilon}{\Delta t}+\frac{1}{2}} Q_{q}(g)(t, x)+O\left(\frac{\Delta t^{3}}{\sqrt{\varepsilon}}\right) .
$$

We conclude by noting that $Q_{q}(g)=\frac{1}{\varepsilon}\left(M_{q}-g_{q}\right)$. 
Proof of Lemma 3.1: When $\widetilde{\varepsilon} \neq-\varepsilon$, we have

$$
\begin{aligned}
Q(f) & =\frac{1}{\varepsilon}\left(\mathcal{M}_{f}-f\right) \\
& =\frac{1}{\varepsilon}\left[\mathcal{M}_{f}-g-\widetilde{\varepsilon} Q(f)\right]
\end{aligned}
$$

which allows to obtain

$$
Q(f)=\frac{1}{\varepsilon+\widetilde{\varepsilon}}\left(\mathcal{M}_{f}-g\right) .
$$

We conclude by noting that $\mathcal{M}_{g}=\mathcal{M}_{f}$.

\subsection{A first LBM scheme}

Let us choose

$$
\begin{cases}c=\frac{\Delta x}{\Delta t}, & \text { (a) } \\ \varepsilon=\frac{v}{c^{2}}, & \text { (b) }\end{cases}
$$

Thus, we have

$$
\varepsilon=C_{d} \Delta t
$$

since $\Delta t:=C_{d} \frac{\Delta x^{2}}{v}\left(C_{d} \geq 0\right)$. We deduce from (21) a first LBM scheme

$$
\left\{\begin{array}{l}
g_{1, i}^{n+1}=g_{1, i+1}^{n}(1-\eta)+M_{1, i+1}^{n} \eta \\
g_{2, i}^{n+1}=g_{2, i-1}^{n}(1-\eta)+M_{2, i-1}^{n} \eta \\
\rho_{i}^{n+1}=g_{1, i}^{n+1}+g_{2, i}^{n+1}
\end{array}\right.
$$

where

$$
\eta:=\frac{1}{C_{d}+\frac{1}{2}}=\frac{1}{\frac{v \Delta t}{\Delta x^{2}}+\frac{1}{2}} .
$$

Let us underline that (32) is equivalent to

$$
v=\Delta t\left(\frac{1}{\eta}-\frac{1}{2}\right) c_{s}^{2}
$$

with $c_{s}=K \Delta x / \Delta t$ (in the studied case, we have $K=1$ ). In the LBM realm, the constant $c_{s}$ is named pseudo sound speed of the lattice. Moreover, since $M_{q}=\frac{g_{1}+g_{2}}{2} \cdot\left[1+(-1)^{q} \frac{\Delta t}{\Delta x} u(x)\right]$, we see that the LBM scheme (31) is equivalent to

$$
\left\{\begin{array}{l}
g_{1, i}^{n+1}=g_{1, i+1}^{n}\left[1-\frac{\eta}{2}\left(1+\frac{\Delta t}{\Delta x} u\left(x_{i+1}\right)\right)\right]+g_{2, i+1}^{n} \frac{\eta}{2}\left(1-\frac{\Delta t}{\Delta x} u\left(x_{i+1}\right)\right) \\
g_{2, i}^{n+1}=g_{2, i-1}^{n}\left[1-\frac{\eta}{2}\left(1-\frac{\Delta t}{\Delta x} u\left(x_{i-1}\right)\right)\right]+g_{1, i-1}^{n} \frac{\eta}{2}\left(1+\frac{\Delta t}{\Delta x} u\left(x_{i-1}\right)\right) \\
\rho_{i}^{n+1}=g_{1, i}^{n+1}+g_{2, i}^{n+1}
\end{array}\right.
$$


and, thus, to

$$
\left\{\begin{array}{l}
g_{1, i}^{n+1}=g_{1, i+1}^{n}\left(1-\frac{\eta}{2}\right)+g_{2, i+1}^{n} \frac{\eta}{2}-\frac{\rho_{i+1}^{n}}{2} \cdot \eta \frac{\Delta t}{\Delta x} u\left(x_{i+1}\right), \\
g_{2, i}^{n+1}=g_{2, i-1}^{n}\left(1-\frac{\eta}{2}\right)+g_{1, i-1}^{n} \frac{\eta}{2}+\frac{\rho_{i-1}^{n}}{2} \cdot \eta \frac{\Delta t}{\Delta x} u\left(x_{i-1}\right), \\
\rho_{i}^{n+1}=g_{1, i}^{n+1}+g_{2, i}^{n+1} .
\end{array}\right.
$$

We will use the formulation (35) in the sequel. When $u(x)=0$, the LBM scheme (35) is identical to the one proposed in $[4,7,32,6]$ to solve the diffusion equation. When $u(x)$ is a constant $u_{0}$, (35) has similarities with the LBM scheme proposed in [18] to solve the advection equation $\partial_{t} \rho+u_{0} \partial_{x} \rho=0$ : this point is studied in Section 8.

Let us remark that $\eta$ defined with (32) belongs to ]0,2] since $C_{d} \geq 0$ : we will use this property in the sequel to obtain convergence results in $L^{\infty}$ and discrete maximum principles. Let us also remark that, due to the relation (20), the LBM scheme (35) is equivalent to the LBM scheme (see Annex B)

$$
\left\{\begin{array}{l}
f_{1, i}^{n+1}=\frac{f_{1, i+1}^{n}\left(16 C_{d}^{2}-1\right)+f_{2, i+1}^{n}\left(4 C_{d}+1\right)+f_{2, i-1}^{n}\left(4 C_{d}-1\right)+f_{1, i-1}^{n}}{16 C_{d}\left(C_{d}+\frac{1}{2}\right)}, \\
f_{2, i}^{n+1}=\frac{f_{1, i+1}^{n}\left(4 C_{d}-1\right)+f_{2, i+1}^{n}+f_{2, i-1}^{n}\left(16 C_{d}^{2}-1\right)+f_{1, i-1}^{n}\left(4 C_{d}+1\right)}{16 C_{d}\left(C_{d}+\frac{1}{2}\right)} \\
\rho_{i}^{n+1}=f_{1, i}^{n+1}+f_{2, i}^{n+1}
\end{array}\right.
$$

when $u(x)=0$ and $C_{d} \neq 0$ (the transformation (20) is not defined when $C_{d}=0$ ). The LBM scheme (36) is not classical. Since (36) is more complex than (35) and is ill-defined when $C_{d}=0$, we will study in the sequel the LBM schemes expressed with the distribution $g_{q}$ and not with the distribution $f_{q}$.

\subsection{A second LBM scheme}

By replacing $\Delta t$ by $-\Delta t$ in (21) and (22), and by choosing again (29), we now deduce from (21) the second LBM scheme

$$
\left\{\begin{array}{l}
g_{1, i+1}^{n-1}=g_{1, i}^{n}(1-\hat{\eta})+M_{1, i}^{n} \hat{\eta}, \\
g_{2, i-1}^{n-1}=g_{2, i}^{n}(1-\hat{\eta})+M_{2, i}^{n} \hat{\eta}, \\
\rho_{i}^{n}=g_{1, i}^{n}+g_{2, i}^{n}
\end{array}\right.
$$

where $\hat{\eta}:=\frac{1}{-C_{d}+\frac{1}{2}}=\frac{1}{-\frac{v \Delta t}{\Delta x^{2}}+\frac{1}{2}}$. We have the following property proved in Annex C:

Property 3.1. When $C_{d} \neq 1 / 2$, the LBM scheme (37) is equivalent to

$$
\left\{\begin{array}{l}
g_{1, i}^{n+1}=g_{1, i+1}^{n}\left[1-\frac{\eta}{2}\left(1+\frac{\Delta t}{\Delta x} u\left(x_{i}\right)\right)\right]+g_{2, i-1}^{n} \frac{\eta}{2}\left(1-\frac{\Delta t}{\Delta x} u\left(x_{i}\right)\right), \\
g_{2, i}^{n+1}=g_{2, i-1}^{n}\left[1-\frac{\eta}{2}\left(1-\frac{\Delta t}{\Delta x} u\left(x_{i}\right)\right)\right]+g_{1, i+1}^{n} \frac{\eta}{2}\left(1+\frac{\Delta t}{\Delta x} u\left(x_{i}\right)\right), \\
\rho_{i}^{n+1}=g_{1, i}^{n+1}+g_{2, i}^{n+1}
\end{array}\right.
$$


where $\eta$ is defined with (32). In the sequel, (38) is named LBM* scheme.

Contrary to the LBM scheme (35), the $\mathrm{LBM}^{*}$ scheme (38) is not classical. Let us note that when $C_{d}=1 / 2$, (37) is not defined since $|\hat{\eta}|$ goes to the infinity when $C_{d}$ goes to $1 / 2$ : this comes from the fact that Lemma 3.1 is valid only when $\widetilde{\varepsilon} \neq-\varepsilon$. Nevertheless, (38) is defined for any $C_{d} \geq 0$ since $\left.\left.\eta \in\right] 0,2\right]$. In the sequel, we will show that the $\mathrm{LBM}^{*}$ scheme (38) is valid for any $C_{d} \geq 0$.

\subsection{Justification of the use of a third order integration formula to obtain the LBM schemes}

We now justify the use of the third order integration formula (28) - which is equivalent to (27) - instead of the second order integration formula (26). This point is important in the LBM realm and justifies the classical formula (33).

\subsubsection{Heuristic justification of the third order formula}

Let us suppose that the quantity $\int_{0}^{\Delta t} Q_{q}(f)\left[t+s, x+(-1)^{q} c s\right] d s$ in (23) is computed with an integration formula of order $m \in \mathbb{N}$. Since $\int_{0}^{\Delta t} Q_{q}(f)\left[t+s, x+(-1)^{q} c s\right] d s$ is of the order of $\Delta t / \sqrt{\varepsilon}$ (see (25)), the error of the integration formula has to be of the order of $\Delta t^{m} / \sqrt{\varepsilon}$ with $m \geq 2$. Moreover, we construct the LBM schemes with $\Delta t=O(\varepsilon)$ (see (30)). To summarize, the error $\Delta t^{m} / \sqrt{\varepsilon}$ is of the order of $\varepsilon^{m-1 / 2}$ and $m$ has to be greater or equal to 2 . We now use the following property:

Property 3.2. Let us suppose that $f_{q}^{\varepsilon}$ is solution of $(18)$ and that $\bar{f}_{q}^{\varepsilon}$ is an approximation of $f_{q}^{\varepsilon}$ which satisfies

$$
\bar{f}_{q}^{\varepsilon}=f_{q}^{\varepsilon}+\varepsilon^{k} \Psi_{q}\left(t, \frac{t}{\varepsilon}, x, \frac{x}{\sqrt{v \varepsilon}}\right)
$$

where $\Psi_{q}\left(t_{1}, t_{2}, x_{1}, x_{2}\right)$ is a $C^{\infty}$-function. Thus, $\bar{\rho}^{\varepsilon}:=\bar{f}_{1}^{\varepsilon}+\bar{f}_{2}^{\varepsilon}$ is solution of

$$
\partial_{t} \bar{\rho}^{\varepsilon}+\partial_{x}\left(u \bar{\rho}^{\varepsilon}\right)=v \partial_{x x}^{2} \bar{\rho}^{\varepsilon}+O(\varepsilon)
$$

when $k \geq 2$.

By taking $k=m-1 / 2$, we deduce from this property that $m-1 / 2$ has to be greater than 2 which implies that $m \geq 3$ since $m \in \mathbb{N}$. This justifies to use the third order integration formula (27) (i.e. $m=3$ ) instead of the second order integration formula (26) (i.e. $m=2$ ). Of course, $m \geq 3$ is only a necessary condition to obtain consistent LBM schemes. It remains to prove that $m=3$ is a sufficient condition, which will be proved in $\S 5$.

Let us note that we impose that $\Psi_{q}$ is a $C^{\infty}$-function to simplify the statement of Property 3.2. Indeed, we could choose $\Psi_{q}$ with less regularity. Moreover, the variables $t / \varepsilon$ and $x / \sqrt{v \varepsilon}$ in $\Psi_{q}$ underlines that this perturbation may act at kinetic scales. Let us also note that the expansion (10) shows that $f_{q}^{\varepsilon}$ may have variations at kinetic scales only at the order $\varepsilon^{2}$, which is coherent with the previous result.

Proof of Property 3.2: Let us suppose that

$$
\bar{f}_{q}^{\varepsilon}=f_{q}^{\varepsilon}+\varepsilon^{k} \Psi_{q}\left(t, \frac{t}{\varepsilon}, x, \frac{x}{\sqrt{v \varepsilon}}\right)
$$


In that case, we have

$$
\left\{\begin{array}{l}
\partial_{t} \bar{\rho}^{\varepsilon}=\partial_{t} \rho^{\varepsilon}+O\left(\varepsilon^{k-1}\right), \\
\partial_{x}\left(u \bar{\rho}^{\varepsilon}\right)=\partial_{x}\left(u \rho^{\varepsilon}\right)+O\left(\varepsilon^{k-1 / 2}\right), \\
\partial_{x x}^{2} \bar{\rho}^{\varepsilon}=\partial_{x x}^{2} \rho^{\varepsilon}+O\left(\varepsilon^{k-1}\right)
\end{array}\right.
$$

which allows to write that

$$
\partial_{t} \bar{\rho}^{\varepsilon}+\partial_{x}\left(u \bar{\rho}^{\varepsilon}\right)-v \partial_{x x}^{2} \bar{\rho}^{\varepsilon}=\partial_{t} \rho^{\varepsilon}+\partial_{x}\left(u \rho^{\varepsilon}\right)-v \partial_{x x}^{2} \rho^{\varepsilon}+O\left(\varepsilon^{k-1}\right) .
$$

Thus, we obtain that

$$
\partial_{t} \bar{\rho}^{\varepsilon}+\partial_{x}\left(u \bar{\rho}^{\varepsilon}\right)=v \partial_{x x}^{2} \bar{\rho}^{\varepsilon}+O(\varepsilon)
$$

when $k \geq 2$ by using (9)

\subsubsection{Concerning the second order integration formula}

We now verify that the LBM schemes built by using (26) (i.e. $m=2$ ) instead of (28) (i.e. $m=3$ ) cannot be consistent with the convection-diffusion equation (19) when $\Delta t=\frac{\Delta x^{2}}{2 v}$. When $u(x)=0$, the LBM and $\mathrm{LBM}^{*}$ schemes using (26) are respectively given by

$$
\left\{\begin{array}{l}
f_{1, i}^{n+1}=f_{1, i+1}^{n}\left(1-\frac{1}{2 C_{d}}\right)+\frac{f_{2, i+1}^{n}}{2 C_{d}} \\
f_{2, i}^{n+1}=f_{2, i-1}^{n}\left(1-\frac{1}{2 C_{d}}\right)+\frac{f_{1, i-1}^{n}}{2 C_{d}} \\
\rho_{i}^{n+1}=f_{1, i}^{n+1}+f_{2, i}^{n+1}
\end{array}\right.
$$

and

$$
\left\{\begin{array}{l}
f_{1, i}^{n+1}=f_{1, i+1}^{n}\left(1-\frac{1}{2\left(1-C_{d}\right)}\right)+\frac{f_{2, i-1}^{n}}{2\left(1-C_{d}\right)} \\
f_{2, i}^{n+1}=f_{2, i-1}^{n}\left(1-\frac{1}{2\left(1-C_{d}\right)}\right)+\frac{f_{1, i+1}^{n}}{2\left(1-C_{d}\right)} \\
\rho_{i}^{n+1}=f_{1, i}^{n+1}+f_{2, i}^{n+1} .
\end{array}\right.
$$

When $C_{d}=1 / 2$ that is to say $\Delta t=\frac{\Delta x^{2}}{2 v}$, formula (39) gives

$$
\left\{\begin{array}{l}
f_{1, i}^{n+1}=f_{2, i+1}^{n}, \\
f_{2, i}^{n+1}=f_{1, i-1}^{n}, \\
\rho_{i}^{n+1}=f_{1, i}^{n+1}+f_{2, i}^{n+1}
\end{array}\right.
$$


which is equivalent to

$$
\left\{\begin{array}{l}
f_{1, i}^{n+1}=f_{1, i}^{n-1}, \\
f_{2, i}^{n+1}=f_{2, i}^{n-1}, \\
\rho_{i}^{n+1}=f_{1, i}^{n+1}+f_{2, i}^{n+1} .
\end{array}\right.
$$

Finally, we obtain that

$$
\rho_{i}^{n+1}=\rho_{i}^{n-1} \quad \text { when } \quad \Delta t=\frac{\Delta x^{2}}{2 v} .
$$

In the same way, we verify that when $C_{d}=1 / 2$, formula (40) implies (41). Relation (41) shows that the LBM schemes (39) and (40) cannot be consistent with the diffusion equation when $\Delta t=\frac{\Delta x^{2}}{2 v}$. By continuity, we deduce that the LBM schemes built by using (26) instead of (28) cannot be consistent with the convection-diffusion equation (19). We underline that Formula (33) is a direct consequence of the use of (28). Moreover, we easily verify that (39) can be seen as the LBM scheme (35) used to solve the diffusion equation $\partial_{t} \rho=\tilde{v} \partial_{x x}^{2} \rho$ with the diffusion coefficient

$$
\tilde{v}=v-\frac{\Delta t}{2} c_{s}^{2}
$$

In [29], a LBM scheme is proposed to solve the incompressible Navier-Stokes system. This LBM scheme is constructed by using the second order formula (26) with $\Delta t>0$ (see (3) in [29]). This explains that the kinematic viscosity in [29] of the PDEs system discretized by the LBM scheme is equal to a diffusion coefficient $\tilde{v}$ similar to (42) instead of $v$ : see (A.16) in [29] (note that $\Delta t=\Delta x=1$ in [29]: see (24)). Nevertheless, the authors in [29] underline the fact that (26) cannot give a good approximation of $f_{q}^{\varepsilon}(t, x)$ when $\varepsilon \ll 1$ although they use (26) (see p. 679 in [29]). In the same way, (40) can be seen as the LBM* scheme (38) used to solve the diffusion equation $\partial_{t} \rho=\hat{v} \partial_{x x}^{2} \rho$ with the diffusion coefficient

$$
\hat{v}=\frac{1}{2}\left(\frac{\Delta t}{2} c_{s}^{2}-v\right) \text {. }
$$

Let us remark that $\tilde{v} \neq \hat{v}$ except when $C_{d}=1 / 2$ (i.e. $\Delta t=\frac{\Delta x^{2}}{2 v}$ ). In this last case, we have $\tilde{v}=\hat{v}=0$ and $\rho_{i}^{n}$ deduced from the LBM schemes (39) or (40) is given by (41), which is coherent with $\partial_{t} \rho=0$.

\section{Link with a finite difference type scheme}

We now prove that the LBM scheme (35) and the $\mathrm{LBM}^{*}$ scheme (38) with the initial condition

$$
\left\{\begin{array}{l}
g_{1, i}^{0}=\rho_{i}^{0} \cdot\left[(1-\alpha)-\beta \frac{\Delta t}{\Delta x} u\left(x_{i}\right)\right], \\
g_{2, i}^{0}=\rho_{i}^{0} \cdot\left[\alpha+\beta \frac{\Delta t}{\Delta x} u\left(x_{i}\right)\right]
\end{array}\right.
$$

(where $(\alpha, \beta) \in \mathbb{R}^{2}$ ) are identical (for the macroscopic quantity $\rho$ ) to the finite difference type scheme

$$
\frac{\rho_{i}^{n+1}-\rho_{i}^{n-1}}{2 \Delta t}+\frac{1}{2 \Delta x}\left[u\left(x_{i+1}\right) \rho_{i+1}^{n}-u\left(x_{i-1}\right) \rho_{i-1}^{n}\right]=\frac{v}{\Delta x^{2}}\left(\rho_{i+1}^{n}-\rho_{i}^{n+1}-\rho_{i}^{n-1}+\rho_{i-1}^{n}\right)
$$


when the first iterate is given by

$$
\rho_{i}^{n=1}:=\xi \rho_{i-1}^{0}+(1-\xi) \rho_{i+1}^{0}-\gamma \frac{\Delta t}{\Delta x}\left[\rho_{i+1}^{0} u\left(x_{i+1}\right)-\rho_{i-1}^{0} u\left(x_{i-1}\right)\right] \quad \text { with }\left\{\begin{array}{l}
\xi=\frac{\eta}{2}+\alpha(1-\eta), \\
\gamma=\frac{\eta}{2}+\beta(1-\eta)
\end{array}\right.
$$

in the case of the LBM scheme (35) (we recall that $\eta$ is defined with (32) and belongs to ]0,2]), and by

$$
\rho_{i}^{n=1}:=\alpha \rho_{i-1}^{0}+(1-\alpha) \rho_{i+1}^{0}-\beta \frac{\Delta t}{\Delta x}\left[\rho_{i+1}^{0} u\left(x_{i+1}\right)-\rho_{i-1}^{0} u\left(x_{i-1}\right)\right]
$$

in the case of the $\mathrm{LBM}^{*}$ scheme (38). We will use this equivalence in $\$ 5$ and $\S 6$ to obtain new stability and convergence results in $L^{\infty}$ and discrete maximum principles. Let us note that $g_{q, i}^{0}$ is equal to the maxwellian

$$
M_{q, i}^{0}:=\frac{\rho_{i}^{0}}{2}\left[1+(-1)^{q} \frac{\Delta t}{\Delta x} u\left(x_{i}\right)\right]
$$

(see (2), (3), (29)(a) and (44)) if and only if $\alpha=\beta=1 / 2$. At last, let us remark that $\xi \in[0,1] \Longleftrightarrow \alpha \in[0,1]$, and $\gamma \in[0,1] \Longleftrightarrow \beta \in[0,1]$.

Let us note that when $u(x)$ is a constant $u_{0}$, the finite difference type scheme (45) is the LFCCDF scheme proposed in [33]: thus, (45) is also named LFCCDF scheme in the sequel. Moreover, when $u(x)=0$, the scheme (45) is the Du Fort-Frankel scheme [35]. Let us also underline that the first iterate (47) - which is equivalent to (46) - is not classical. For example, the first iterate in [35] is defined with the classical scheme

$$
\frac{\rho_{i}^{n=1}-\rho_{i}^{n}}{\Delta t}=\frac{v}{\Delta x^{2}}\left(\rho_{i+1}^{0}-2 \rho_{i}^{0}+\rho_{i-1}^{0}\right)
$$

(the first iterate is not defined in [33]). The first iterate (47) (resp. (46)) is essential, firstly, to obtain the equivalence between the LBM scheme (38) (resp. (35)) with (45) and, secondly, to obtain convergence results in $L^{\infty}$ and discrete maximum principles for any $C_{d} \geq 0$ (see $\$ 5$ and $\S 6$ ). At last, let us note that the link between the LBM scheme (35) with the Du Fort-Frankel scheme was firstly cited in [32] in the case of periodic boundary conditions.

The proof of lemmae proposed in this section are written in Annex D.

\subsection{Periodic boundary conditions for the convection-diffusion equation}

We have the following result:

Lemma 4.1. In the periodic case:

i) The LBM scheme (35)(44) is identical to the LFCCDF scheme (45)(46).

ii) The LBM* scheme (38)(44) is identical to the LFCCDF scheme (45)(47).

iii) The LBM scheme (35)(44) and the $L B M^{*}$ scheme (38)(44) are identical if and only if $\alpha=\beta=\frac{1}{2}$.

We underline that when the LBM schemes (35)(44) and (38)(44) are said to be identical, this means that they give the same $\rho_{i}^{n}$ for any $(i, n)$. Nevertheless, this does not mean that they give the same $g_{i}^{n}$ for any $(i, n)$. 


\subsection{Neumann boundary conditions for the diffusion equation}

We suppose that $u(x)=0$. To apply the Neumann boundary condition $\partial_{x} \rho\left(t, x_{\min }\right)=0$ in $x=x_{\min }$, we define the mesh $x_{i}=x_{\min }+\left(i-\frac{1}{2}\right) \Delta x(i=0, \ldots)$. For the sake of simplicity, we forget the boundary condition in $x=x_{\max }$. The LBM scheme (35) is now given by

$$
\left\{\begin{array}{l}
g_{1, i}^{n+1}=g_{1, i+1}^{n}\left(1-\frac{\eta}{2}\right)+g_{2, i+1}^{n} \frac{\eta}{2} \\
g_{2, i}^{n+1}=g_{2, i-1}^{n}\left(1-\frac{\eta}{2}\right)+g_{1, i-1}^{n} \frac{\eta}{2} \\
\rho_{i}^{n+1}=g_{1, i}^{n+1}+g_{2, i}^{n+1} .
\end{array}\right.
$$

And, the $\mathrm{LBM}^{*}$ scheme (38) is now given by

$$
\left\{\begin{array}{l}
g_{1, i}^{n+1}=g_{1, i+1}^{n}\left(1-\frac{\eta}{2}\right)+g_{2, i-1}^{n} \frac{\eta}{2} \\
g_{2, i}^{n+1}=g_{2, i-1}^{n}\left(1-\frac{\eta}{2}\right)+g_{1, i+1}^{n} \frac{\eta}{2} \\
\rho_{i}^{n+1}=g_{1, i}^{n+1}+g_{2, i}^{n+1} .
\end{array}\right.
$$

Moreover, the initial condition (44) is now given by

$$
\begin{cases}g_{1, i}^{0}=(1-\alpha) \rho_{i}^{0}, & \text { (a) } \\ g_{2, i}^{0}=\alpha \rho_{i}^{0} . & \text { (b) }\end{cases}
$$

We have the following result:

Lemma 4.2. The LBM $^{*}$ scheme (51)(52) with the boundary conditions

$$
\begin{cases}g_{2, i=0}^{n+1}=g_{1, i=1}^{n+1}+\left(g_{2, i=0}^{n}-g_{1, i=1}^{n}\right)(1-\eta), & (a) \\ g_{2, i=0}^{n=0}=\alpha \rho_{i=1}^{n=0} & (b)\end{cases}
$$

is identical to the Du Fort-Frankel scheme

$$
\forall i \geq 1, \forall n \geq 1: \quad \frac{\rho_{i}^{n+1}-\rho_{i}^{n-1}}{2 \Delta t}=\frac{v}{\Delta x^{2}}\left(\rho_{i+1}^{n}-\rho_{i}^{n+1}-\rho_{i}^{n-1}+\rho_{i-1}^{n}\right)
$$

with the discrete Neumann boundary condition

$$
\forall n \geq 0: \quad \rho_{i=0}^{n}=\rho_{i=1}^{n}
$$

when the first iterate is given by

$$
\forall i \geq 1: \quad \rho_{i}^{n=1}=\alpha \rho_{i-1}^{0}+(1-\alpha) \rho_{i+1}^{0} \quad \text { where } \quad \alpha \in \mathbb{R} .
$$


We have the same result for the LBM scheme (50)(52) by replacing the boundary conditions (53) with

$$
\begin{cases}g_{1, i=0}^{n+1}=g_{2, i=1}^{n+1}+\left(g_{1, i=1}^{n}-g_{2, i=0}^{n}\right)(1-\eta), & \text { (a) } \\ g_{2, i=0}^{n+1}=g_{1, i=1}^{n+1}+\left(g_{2, i=0}^{n}-g_{1, i=1}^{n}\right)(1-\eta), & \text { (b) } \\ g_{1, i=0}^{n=0}=(1-\alpha) \rho_{i=1}^{n=0}, & \text { (c) } \\ g_{2, i=0}^{n=0}=\alpha \rho_{i=1}^{n=0} & \text { (d) }\end{cases}
$$

and by replacing the first iterate (56) with

$$
\forall i \geq 1: \quad \rho_{i}^{n=1}:=\xi \rho_{i-1}^{0}+(1-\xi) \rho_{i+1}^{0} \quad \text { where } \quad \xi=\frac{\eta}{2}+\alpha(1-\eta) .
$$

Thus, the LBM scheme (50)(52)(57) and the LBM* scheme (51)(52)(53) are identical if and only if $\alpha=\frac{1}{2}$.

When $\alpha \neq \frac{1}{2}$, the boundary conditions (53) and (57) are not classical in the framework of the LBM schemes. Nevertheless, when $\alpha=\frac{1}{2}$ that is to say when

$$
g_{1, i}^{0}=g_{2, i}^{0}=\frac{\rho_{i}^{0}}{2}
$$

the boundary conditions (53) and (57) are respectively equivalent to

$$
\forall n \geq 0: \quad g_{2, i=0}^{n}=g_{1, i=1}^{n}
$$

and

$$
\forall n \geq 0: \quad g_{1, i=0}^{n}=g_{2, i=1}^{n} \quad \text { and } \quad g_{2, i=0}^{n}=g_{1, i=1}^{n} .
$$

The boundary conditions (60) and (61) are classical in the framework of the LBM schemes: they are an approximation at the discrete level on $x_{\min }:=\frac{1}{2}\left(x_{i=0}+x_{i=1}\right)$ of the bounce-back boundary conditions [38] which corresponds to

$$
\forall t \geq 0: \quad g_{1}\left(t, x=x_{\min }\right)=g_{2}\left(t, x=x_{\min }\right)
$$

in the studied case. For example, (61) is equivalent to the Neumann boundary condition proposed in $[6,39]$ (more precisely, (21) in [6] is equivalent to (59), and we easily show that (28) with $q_{b}=0$ in [6] is equivalent to (61) as soon as (61) is satisfied with $n=0$ ). We can justify (62) - and, thus, (60) and (61) - with the following heuristic argument. When $\partial_{x} \rho\left(t, x=x_{\min }\right)=0$, the asymptotic expansion (13) gives

$$
\forall t \geq 0, \forall q \in\{1,2\}: \quad f_{q}\left(t, x=x_{\min }\right)=\frac{\rho}{2}\left(t, x=x_{\min }\right)+O\left(\varepsilon^{3 / 2}\right) .
$$

This incites us to impose $f_{1}\left(t, x=x_{\min }\right)=f_{2}\left(t, x=x_{\min }\right)=\frac{\rho}{2}\left(t, x=x_{\min }\right)$ that is to say

$$
\forall t \geq 0, \forall q \in\{1,2\}: \quad g_{q}\left(t, x=x_{\min }\right)=\frac{\rho}{2}\left(t, x=x_{\min }\right)
$$

by using the transformation (20), which is equivalent to (62) since $g_{1}+g_{2}=\rho$. Unfortunatly, this justification cannot be satisfactory from a theoretical point of view. Indeed, the asymptotic expansion (13) is valid with periodic boundary condition on $\partial \Omega$. With other boundary conditions (such as Neumann and Dirichlet boundary conditions), we would have to study a Knudsen layer created by the discrete velocity kinetic system (11) in the vicinity of $\partial \Omega$, which would make much more difficult the analysis ([40] could be a starting point). Nevertheless, Lemma 4.2 justifies (60) and (61) through the equivalence with the Du FortFrankel scheme. 


\subsection{Dirichlet boundary conditions for the diffusion equation}

We also suppose that $u(x)=0$ and we also forget the boundary conditions in $x_{\max }$ for the sake of simplicity. To apply the Dirichlet boundary condition $\rho\left(t, x_{\min }\right)=\rho_{x_{\min }}$, we now define the mesh $x_{i}=$ $x_{\min }+i \Delta x(i=0, \ldots)$.

We have the following result:

Lemma 4.3. The LBM* scheme (51)(52) with the boundary conditions

$$
\begin{cases}g_{2, i=0}^{n+1}=\frac{\rho_{x_{\min }}}{2}+\left(\frac{\rho_{x_{\min }}}{2}-g_{1, i=1}^{n}\right)(1-\eta), & \text { (a) } \\ g_{2, i=0}^{n=0}=\alpha \rho_{x_{\min }} & (b)\end{cases}
$$

is identical to the Du Fort-Frankel scheme (54) with the Dirichlet boundary condition

$$
\forall n \geq 0: \quad \rho_{i=0}^{n}=\rho_{x_{\min }}
$$

when the first iterate is given by (56). We have the same result for the LBM scheme (50)(52) by replacing the boundary conditions (63) with

$$
\begin{cases}g_{1, i=0}^{n+1}=g_{1, i=1}^{n}\left(1-\frac{\eta}{2}\right)+g_{2, i=1}^{n} \frac{\eta}{2}, & \text { (a) } \\ g_{2, i=0}^{n+1}=\rho_{x_{\min }}-g_{1, i=0}^{n+1}, & (b) \\ g_{1, i=0}^{n=0}=(1-\alpha) \rho_{x_{\min }}, & (c) \\ g_{2, i=0}^{n=0}=\alpha \rho_{x_{\min }} & (d)\end{cases}
$$

and by replacing the first iterate (56) with (58). Thus, the LBM scheme (50)(52)(65) and the LBM* scheme (51)(52)(63) are identical if and only if $\alpha=\frac{1}{2}$.

The boundary condition (65) is identical to the one proposed in [6] when $\alpha=1 / 2$ (see (21) and (25) in [6]). Let us also note that (65) is equivalent to

$$
\left\{\begin{array}{l}
g_{1, i=0}^{n+1}=\frac{\rho_{i=1}^{n}}{2}+\left(\frac{\rho_{i=1}^{n}}{2}-g_{2, i=1}^{n}\right)(1-\eta), \\
g_{2, i=0}^{n+1}=\rho_{x_{\min }}-\frac{\rho_{i=1}^{n}}{2}+\left(\frac{\rho_{i=1}^{n}}{2}-g_{1, i=1}^{n}\right)(1-\eta), \\
g_{1, i=0}^{n=0}=(1-\alpha) \rho_{x_{\min }} \\
g_{2, i=0}^{n=0}=\alpha \rho_{x_{\min }}
\end{array}\right.
$$

which underlines that (65)(b) is not exactly equal to (63)(a). 
We may think that the boundary condition (63) for the LBM* scheme (51) should be replaced by

$$
\forall n \geq 0: \quad g_{2, i=0}^{n}=\frac{\rho_{x_{\min }}}{2},
$$

and that the boundary condition (65) for the LBM scheme (50) should be replaced by

$$
\forall n \geq 0: \quad g_{1, i=0}^{n}=g_{2, i=0}^{n}=\frac{\rho_{x_{\min }}}{2}
$$

since (67) and (68) seem to be more simple. In \$6.2, we will explain why we should replace (63) (resp. (65)) by (67) (resp. (68)) when the number of cells is low even if we lose the equivalence with the Du Fort-Frankel (54) scheme when we use (67) (resp. (68)).

\section{Stability and convergence in $L^{\infty}$ for the LBM schemes}

We now prove stability and convergence results in $L^{\infty}$. We recall that the time step $\Delta t \geq 0$ and $\left.\left.\eta \in\right] 0,2\right]$ are respectively defined with (17) and (32).

The proof of propositions proposed in this section are written in Annex E.

\subsection{Periodic boundary conditions for the advection-diffusion equation with constant velocity}

We are now interested in the advection-diffusion equation

$$
\left\{\begin{array}{l}
\partial_{t} \rho+u_{0} \partial_{x} \rho=v \partial_{x x}^{2} \rho, \\
\rho(0, x)=\rho^{0}(x)
\end{array}\right.
$$

where $u_{0} \in \mathbb{R}$ is a constant velocity. We prove the following result:

Proposition 5.1. Let us suppose that the boundary conditions are periodic and that $u(x)$ is constant and equal to $u_{0}$. Then, under the condition

$$
\begin{cases}\left.\Delta x \in] 0, \frac{\min \left(1,2 C_{d}\right)}{C_{d}} \cdot \frac{v}{\left|u_{0}\right|}\right], \quad(a) \\ \alpha \in[0,1], \quad(b) \\ \beta=0 \quad \text { or } \beta=\min (1-\alpha, \alpha), \quad(c)\end{cases}
$$

for any $C_{d}>0$ :

i) The LBM scheme (35)(44) and the $L B M^{*}$ scheme (38)(44) converge in $L^{\infty}$ and verify the discrete maximum principle

$$
\forall i, \forall n \geq 0: \quad \min _{j} \rho_{j}^{0} \leq \rho_{i}^{n} \leq \max _{j} \rho_{j}^{0} .
$$

ii) The LFCCDF scheme (45)(47) converges in $L^{\infty}$ and verifies (71).

iii) The error of the schemes (35)(44), (38)(44) and (45)(47) is of order $\Delta x^{2}$ if and only if $\alpha=\beta=\frac{1}{2}$. 
The case $C_{d}=0$ is particular since the schemes satisfy $\rho_{i}^{n+2}=\rho_{i}^{n}$ when $C_{d}=0$ : see Lemma 7.1. Nevertheless, this property allows to write that the schemes are stable in $L^{\infty}$ for any $\Delta x>0, \alpha \in \mathbb{R}$ and $\beta \in \mathbb{R}$, and for any non-constant periodic velocity $u(x)$ when $C_{d}=0$. Moreover, when $u(x)$ is also a constant $u_{0}$, it is proved in [33] with a von Neumann stability analysis that the LFCCDF scheme (45) is stable in $L^{2}$ under the CFL condition $\Delta t \leq \frac{\Delta x}{\left|u_{0}\right|}$ (see (16c) in [33]). Here, we obtain the stability in $L^{\infty}$ for any $\Delta t>0$ when $\Delta x$ is enough small (see (70)(a), which can be seen as a low Reynolds number condition). At last, let us note that when $u_{0}=0$, we obtain the convergence in $L^{\infty}$ for any $C_{d} \geq 0$, for any $\Delta x>0$ and for any $\alpha \in \mathbb{R}$ : see below Proposition 5.2.

\subsection{Periodic, Neumann and Dirichlet boundary conditions for the diffusion equation}

We are now interested in the diffusion equation

$$
\left\{\begin{array}{l}
\partial_{t} \rho=v \partial_{x x}^{2} \rho, \\
\rho(0, x)=\rho^{0}(x) .
\end{array}\right.
$$

In the periodic, Neumann and Dirichlet cases, the LBM scheme (50)(52) and the LBM* scheme (51)(52) are equivalent to the Du Fort-Frankel scheme (54) when the first iterate is given by (56) that is to say by

$$
\rho_{i}^{n=1}:=\alpha \rho_{i-1}^{0}+(1-\alpha) \rho_{i+1}^{0} \quad \text { where } \quad \alpha \in \mathbb{R}
$$

(see Lemmae 4.1, 4.2 and 4.3). This will allow us to obtain for any $C_{d} \geq 0$, firstly, the stability in $L^{\infty}$ and, secondly, the convergence in $L^{\infty}$.

For the sake of simplicity, we forget the boundary condition in $x=x_{\max }$ in the cases of Neumann and Dirichlet boundary conditions. We have the following result:

\section{Proposition 5.2.}

i) The LBM ${ }^{*}$ scheme (51)(52) with periodic, Neumann or Dirichlet boundary conditions converge in $L^{\infty}$ and verifies:

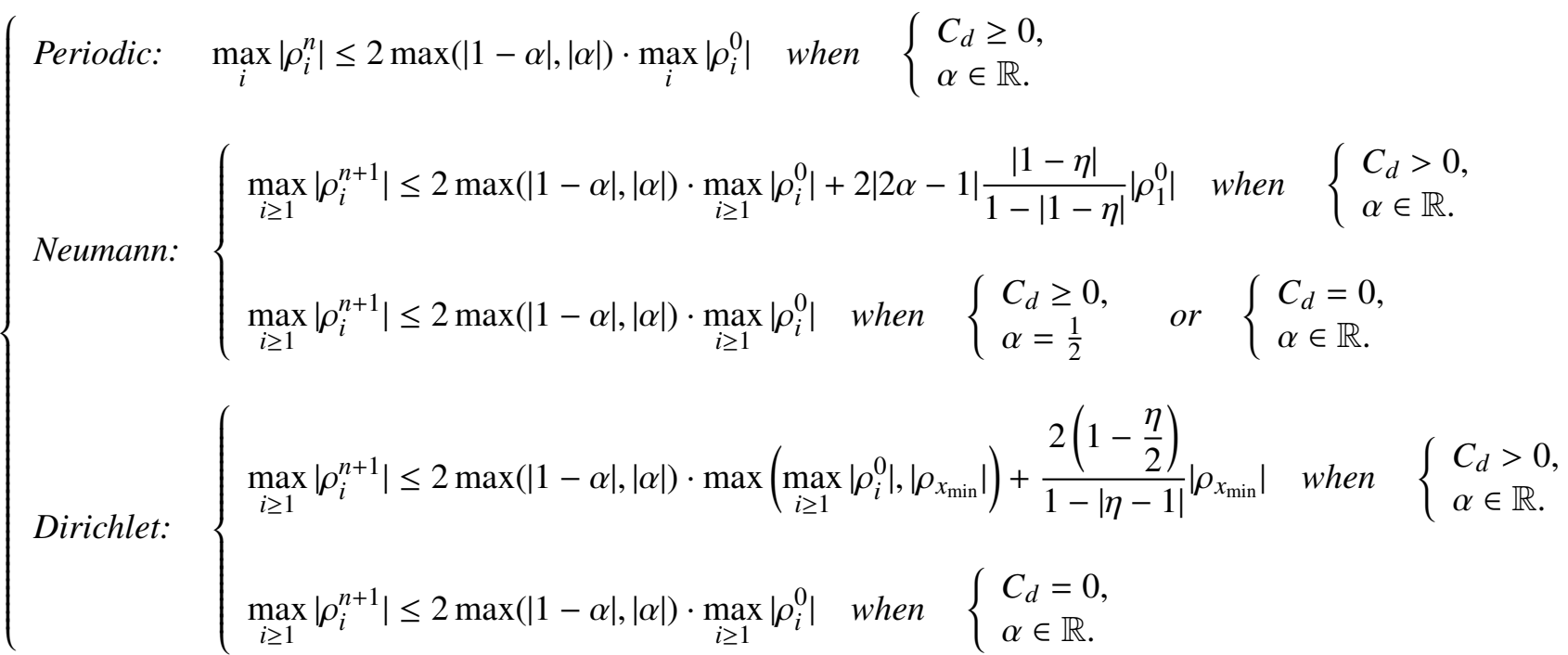


We have the same results for the LBM scheme (50)(52) by replacing $\alpha$ with $\xi=\frac{\eta}{2}+\alpha(1-\eta)$ in (73).

ii) The Du Fort-Frankel scheme (54) with periodic, Neumann or Dirichlet boundary conditions converges in $L^{\infty}$ when the first iterate is given by (56), and verifies (73).

iii) The error of the LBM schemes and of the Du Fort-Frankel scheme is of order $\Delta x$ when $\alpha \neq \frac{1}{2}$ and is of order $\Delta x^{2}$ if and only if $\alpha=\frac{1}{2}$.

It is known since 1953 that the Du Fort-Frankel scheme with periodic boundary conditions is stable - and, thus, is convergent - in $L^{2}$ for any $C_{d} \geq 0$ (this result is obtained with a von Neumann stability analysis). Since the Du Fort-Frankel scheme may be written with

$$
\rho_{i}^{n+1}=\rho_{i+1}^{n}\left(1-\frac{\eta}{2}\right)+\rho_{i-1}^{n}\left(1-\frac{\eta}{2}\right)+\rho_{i}^{n-1}(\eta-1)
$$

(see (155) with $u(x)=0$ ), we have also the stability in $L^{\infty}$ under the stability condition $\eta \in[1,2]$ that is to say when $0 \leq C_{d} \leq 1 / 2$ (for a reasonable choice of the first iterate: with (49) for example). Here, we obtain the stability in $L^{\infty}$ of the Du Fort-Frankel scheme - and, thus, the convergence in $L^{\infty}$ - for any $C_{d} \geq 0$ when the first iterate is defined with (56).

Moreover, when the boundary conditions is a Dirichlet boundary condition in $x=x_{\min }$ and a Neumann boundary condition in $x=x_{\max }$, due to the linearity of the scheme, we easily deduce from the proof of Proposition 5.2 that we simply have to replace $(73)(b, c)$ by

$$
\max _{1 \leq i \leq N}\left|\rho_{i}^{n+1}\right| \leq 2 \max (|1-\alpha|,|\alpha|) \cdot \max \left(\max _{1 \leq i \leq N}\left|\rho_{i}^{0}\right|,\left|\rho_{x_{\min }}\right|\right)+\frac{2\left(1-\frac{\eta}{2}\right)}{1-|\eta-1|}\left|\rho_{x_{\min }}\right|+2|2 \alpha-1| \frac{|1-\eta|}{1-|1-\eta|}\left|\rho_{N}^{0}\right|
$$

(when $C_{d}>0$ and $\alpha \in \mathbb{R}$ ) where $N$ is the number of cells. The other possible cases are similar. At last, let us remark that $(73)(\mathrm{b}, \mathrm{c})$ with $C_{d}=0$ (that is to say when $\left.\Delta t=0\right)$ is studied because the schemes do not verify $\rho_{i}^{n}=\rho_{i}^{0}$ when $C_{d}=0$ : we will discuss about this question in $\S 7.1$.

\section{Discrete maximum principles for the LBM schemes applied to the diffusion equation}

It is easy to obtain a discrete maximum principle for the Du Fort-Frankel scheme (54) when $C_{d} \in\left[0, \frac{1}{2}\right]$ (we recall that $\Delta t:=C_{d} \frac{\Delta x^{2}}{v}$ ). Indeed, this scheme can be rewritten with

$$
\rho_{i}^{n+1}=\rho_{i+1}^{n}\left(1-\frac{\eta}{2}\right)+\rho_{i-1}^{n}\left(1-\frac{\eta}{2}\right)+\rho_{i}^{n-1}(\eta-1)
$$

(see (155) with $u(x)=0$ ) which allows to obtain discrete maximum principles when $\eta \in[1,2]$ that is to say when $C_{d} \in\left[0, \frac{1}{2}\right]$ since $\eta=\frac{1}{C_{d}+\frac{1}{2}}$.

On the other side, we proved in Proposition 5.2 the stability in $L^{\infty}$ for any $C_{d} \geq 0$. Thus, we may think that we could also obtain discrete maximum principles for any $C_{d} \geq 0$. Unfortunatly, Inequalities (73) do not allow to conclude. Moreover, when $C_{d}$ goes to the infinity (that is to say when $\eta=0$ ), the constants in the right hand sides of (73)(b,c) go to the infinity (except when $\alpha=\frac{1}{2}$ in the Neumann case). 
Moreover, the LBM schemes are often applied in complex porous media which may contain areas where the number of cells is low. Thus, it is important to obtain discrete maximum principles (to preserve for example the positivity of the temperature or of the mass fraction when these quantities are solution of a diffusion equation) and, by the same time, to have $C_{d}$ at least greater than $\frac{1}{2}$ to justify the use of the LBM schemes (or of the Du Fort-Frankel scheme) instead of the classical three-points finite difference type scheme (which satisfies discrete maximum principles when $\left.C_{d} \in\left[0, \frac{1}{2}\right]\right)$.

We show below that it is possible to obtain discrete maximum principles for any $C_{d} \geq 0$ with the LBM scheme (50)(52) and with the LBM* scheme (51)(52) in the periodic and Neumann cases. Thus, this is also the case for the Du Fort-Frankel scheme. In the Dirichlet case, we will show that we have to change the boundary conditions (63) and (65) to obtain a discrete maximum principle for any $C_{d} \geq 0$. Unfortunatly, we will lose in that case the equivalence between the LBM schemes and the Du Fort-Frankel scheme, and the error of the LBM schemes will not be of order $\Delta x^{2}$ but should be of order $\Delta x$. As a consequence, this modified Dirichlet boundary conditions will have to be applied only in areas where the number of cells is low. This point underlines the utility of the LBM schemes to obtain robust schemes.

As before, for the sake of simplicity, we forget the boundary condition in $x=x_{\max }$ in the cases of Neumann and Dirichlet boundary conditions.

The proof of the results proposed in this section are written in Annex F.

\subsection{Periodic and Neumann boundary conditions}

We have the following result:

Proposition 6.1. For any $C_{d} \geq 0$ :

i) When $\alpha \in[0,1]$, the LBM scheme (50)(52) and the LBM* scheme (51)(52) with periodic boundary conditions verify the discrete maximum principle

$$
\forall i, \forall n \geq 0: \quad \min _{j} \rho_{j}^{0} \leq \rho_{i}^{n} \leq \max _{j} \rho_{j}^{0} .
$$

ii) When $\alpha=\frac{1}{2}$, the LBM scheme (50)(52) and the LBM* scheme (51)(52) with Neumann boundary conditions verify the discrete maximum principle

$$
\forall i \geq 1, \forall n \geq 0: \quad \min _{j \geq 1} \rho_{j}^{0} \leq \rho_{i}^{n} \leq \max _{j \geq 1} \rho_{j}^{0} .
$$

Thus, this is also the case for the Du Fort-Frankel scheme (54) with periodic or Neumann boundary condition when the first iterate is given by (56).

Let us note that the Du Fort-Frankel scheme is equivalent to

$$
\rho_{i}^{n+1}=\rho_{i+1}^{n}\left(1-\frac{\eta}{2}\right)+\rho_{i-1}^{n}\left(1-\frac{\eta}{2}\right)+\rho_{i}^{n-1}(\eta-1)
$$


(see (155) with $u(x)=0)$. Thus, when $\rho_{i}^{n=1}:=\alpha \rho_{i-1}^{0}+(1-\alpha) \rho_{i+1}^{0}$, we obtain

$$
\begin{aligned}
\rho_{i}^{2} & =\left[\alpha \rho_{i}^{0}+(1-\alpha) \rho_{i+2}^{0}\right]\left(1-\frac{\eta}{2}\right)+\left[\alpha \rho_{i-2}^{0}+(1-\alpha) \rho_{i}^{0}\right]\left(1-\frac{\eta}{2}\right)+\rho_{i}^{0}(\eta-1) \\
& =\left[\alpha \rho_{i-2}^{0}+(1-\alpha) \rho_{i+2}^{0}\right]\left(1-\frac{\eta}{2}\right)+\rho_{i}^{0} \frac{\eta}{2} .
\end{aligned}
$$

This proves that $\min _{j} \rho_{j}^{0} \leq \rho_{i}^{2} \leq \max _{j} \rho_{j}^{0}$ for any $C_{d} \geq 0$ in the periodic case when $\alpha \in[0,1]$. Nevertheless, it is a priori more difficult to obtain a similar result for $\rho_{i}^{n \geq 3}$ without using the equivalence between the LBM $^{*}$ scheme and the Du Fort-Frankel scheme. This remark shows that the LBM schemes may also be seen as a numerical analysis tool to study properties of classical finite difference schemes.

\subsection{Modified Dirichlet boundary conditions}

We have the following result:

Lemma 6.1. For any $C_{d} \in\left[0, \frac{1}{2}\right]$ and when $\alpha=\frac{1}{2}$, the LBM scheme (50)(52)(65) and the LBM* scheme (51)(52)(63) verify the maximum principle

$$
\forall i \geq 1, \forall n \geq 0: \quad \min \left(\rho_{x_{\min }}, \min _{j \geq 1} \rho_{j}^{0}\right) \leq \rho_{i}^{n} \leq \max \left(\rho_{x_{\min }}, \max _{j \geq 1} \rho_{j}^{0}\right) .
$$

Thus, this is also the case for the Du Fort-Frankel scheme (54) with Dirichlet boundary conditions when the first iterate is given by (56).

Lemma 6.1 is less interesting than Proposition 6.1 since the discrete maximum principle (76) is satisfied under the condition $C_{d} \in\left[0, \frac{1}{2}\right]$. Nevertheless, by modifying the Dirichlet boundary conditions (63) and (65), we obtain the following result:

Proposition 6.2. For any $C_{d} \geq 0$ and when $\alpha=\frac{1}{2}$, the LBM* scheme (51)(52) with the modified Dirichlet boundary condition

$$
\forall n \geq 0: \quad g_{2, i=0}^{n}=\frac{\rho_{x_{\min }}}{2}
$$

verifies the maximum principle

$$
\forall i \geq 1, \forall n \geq 0: \quad \min \left(\rho_{x_{\min }}, \min _{j \geq 1} \rho_{j}^{0}\right) \leq \rho_{i}^{n} \leq \max \left(\rho_{x_{\min }}, \max _{j \geq 1} \rho_{j}^{0}\right) .
$$

We have the same result for the LBM scheme (50)(52) with the modified Dirichlet boundary conditions

$$
\forall n \geq 0: \quad g_{1, i=0}^{n}=g_{2, i=0}^{n}=\frac{\rho_{x_{\min }}}{2} .
$$

Let us remark that when $C_{d}=\alpha=\frac{1}{2}$, the modified Dirichlet boundary condition (77) is equivalent to the Dirichlet boundary conditions (63) firstly proposed and which make equivalent the LBM* scheme and the Du Fort-Frankel scheme (see Lemma 4.2). This is coherent with the fact that it is possible to easily prove that the Du Fort-Frankel scheme with Dirichlet boundary condition satisfies the maximum principle when $\alpha \in[0,1]$ and $0 \leq C_{d} \leq 1 / 2$. 
By supposing that Expansion (13) is valid near the boundary $x=x_{\min }-$ which is not proven since the boundary conditions are periodic in Corollary $2.1-$, we obtain that

$$
\frac{\rho}{2}-f_{q}=O(\sqrt{\Delta t})=O(\Delta x)
$$

since $\varepsilon=O(\Delta t)(c f . \S 3.2)$ and $\Delta t=O\left(\Delta x^{2}\right)$. In the same way, we obtain that

$$
\frac{\Delta t}{2} Q_{q}(f)=O(\Delta x)
$$

As a consequence, by using (20) and since $\rho_{x_{\min }}-\rho_{i=1}^{n}=O(\Delta x)$, we obtain that

$$
\left(\frac{\rho_{x_{\min }}}{2}-g_{1, i=1}^{n}\right)(1-\eta)=(1-\eta) O(\Delta x) .
$$

Although this term goes to zero when $\Delta x$ goes to zero, it is important in the Dirichlet boundary conditions (63) to obtain the equivalence between the LBM* scheme (51)(52)(63) and the Du Fort-Frankel scheme (54)(56) with Dirichlet boundary condition. Moreover, when $\alpha=\frac{1}{2}$, the error of the $\mathrm{LBM}^{*}$ scheme (51)(52)(63) is of the order of $\Delta x^{2}$ (see point iii of Proposition 5.2).

Thus, when we replace (63) by (77) in the LBM* scheme:

- The error of the $\mathrm{LBM}^{*}$ scheme with $\alpha=\frac{1}{2}$ should be of the order of $\Delta x$ instead of $\Delta x^{2}$.

- Near the boundary $x=x_{\min }$, we lose the equivalence between the $\mathrm{LBM}^{*}$ scheme and the Du FortFrankel scheme. This avoids to obtain the convergence in $L^{\infty}$ for any $C_{d} \geq 0$ of the $\mathrm{LBM}^{*}$ scheme with modified Dirichlet boundary condition by using the Lax Theorem.

Of course, we have the same remarks when we replace (65) by (79) in the LBM scheme. Nevertheless, Proposition 6.2, estimation (80) (which remains to be proven) and the fact that the equivalence with the $\mathrm{Du}$ Fort-Frankel scheme remains valid far from the boundary $x=x_{\min }$ incite us to conjecture the following result:

Conjecture 6.1. For any $C_{d} \geq 0$ and when $\alpha=\frac{1}{2}$, the $L B M^{*}$ scheme (51)(52) with the modified Dirichlet boundary conditions (77) converges in $L^{\infty}$ with an error of the order of $\Delta x$. We have the same result for the LBM scheme (50)(52) with the modified Dirichlet boundary conditions (79).

Numerical results proposed in $§ 10.1$ will justify this conjecture.

\section{Limitations of the LBM schemes}

\subsection{Preservation of the initial condition}

We have the following result:

Lemma 7.1. When $C_{d}=0$, the LBM* scheme (38)(44) with periodic boundary conditions preserve the initial condition in the sense

$$
\forall i, \forall n \geq 0: \quad \rho_{i}^{n+2}=\rho_{i}^{n} .
$$

Thus, this is also the case for the LBM scheme (35)(44) and for the LFCCDF scheme (45) when the first iterate is given by (47). 
In the case of the diffusion equation with Neumann or Dirichlet boundary conditions, we obtain the following lemma:

Lemma 7.2. When $C_{d}=0$, the $L B M^{*}$ scheme (51)(52) with periodic, Neumann, Dirichlet or modified Dirichlet boundary conditions preserve the initial condition in the sense

$$
\forall i, \forall n \geq 0: \quad \rho_{i}^{n+2}=\rho_{i}^{n} .
$$

Thus, this is also the case for the LBM scheme (50)(52) and for the Du Fort-Frankel scheme (54) when the first iterate is given by (56).

These lemmae show that the proposed LBM schemes do not satisfy $\rho_{i}^{n+1}=\rho_{i}^{n}$ when $C_{d}=0$ i.e. when $\Delta t=0$. Nevertheless, we deduce from (47) that $\rho_{i}^{1}-\rho_{\text {exact }}\left(\Delta t, x_{i}\right)=O\left(\Delta x^{\beta}\right)$ with $\beta=1$ when $\alpha \neq \frac{1}{2}$ and with $\beta=2$ when $\alpha=\frac{1}{2}$. Thus, the proposed LBM schemes preserve the initial condition when $\Delta t=0$ with an error of order $\Delta x^{\beta}$. As a consequence, we cannot say that this property is an important limitation of the proposed LBM schemes.

Proof of Lemma 7.1: When $C_{d}=0$, we deduce from the LBM* scheme (38) that

$$
g_{1, i}^{n+1}=g_{2, i-1}^{n} \quad \text { and } \quad g_{2, i}^{n+1}=g_{1, i+1}^{n} .
$$

As a consequence, we have

$$
g_{1, i}^{n+1}=g_{1, i}^{n-1} \quad \text { and } \quad g_{2, i}^{n+1}=g_{2, i}^{n-1}
$$

which concludes the proof. We obtain the result for the LBM scheme and for the finite difference type scheme (45) by using Lemma 4.1

Proof of Lemma 7.2: The proof in the case of periodic boundary conditions is a direct application of Lemma 7.1.

- The case of Neumann boundary conditions:

For the $\mathrm{LBM}^{*}$ scheme, we deduce from (53)(a) that $g_{2,0}^{n+1}=g_{1,1}^{n+1}-\left(g_{2,0}^{n}-g_{1,1}^{n}\right)$ when $C_{d}=0$. On the other side, we have also $g_{1,1}^{n+1}=g_{2,0}^{n}$ for any $i \geq 1$ by using (51) with $C_{d}=0$. Thus, we obtain $g_{2,0}^{n+1}=g_{1,1}^{n}$ that is to say $g_{2,0}^{n+1}=g_{1,1}^{n}=g_{2,0}^{n-1}$ which allows to obtain the result. We obtain the result for the LBM and Du Fort-Frankel schemes by applying Lemma 4.2.

- The case of Dirichlet boundary conditions:

For the $\mathrm{LBM}^{*}$ scheme, we deduce from (63)(a) that $g_{2,0}^{n+1}=g_{1,1}^{n}$ when $C_{d}=0$. We conclude as in the Neumann case. We obtain the result for the LBM and Du Fort-Frankel schemes by applying Lemma 4.3.

- The case of modified Dirichlet boundary conditions for the LBM and LBM* schemes:

Since the boundary conditions (77) and (79) do not depend on the time, we obtain the result as in the other cases. 


\subsection{Consistency condition}

We proved in Lemma 4.1 that the LBM scheme (35)(44) and the LBM* scheme (38)(44) are equivalent to the finite difference type scheme (45) (when the first iterate is defined with (45)(47)). On the other side, we know that the consistency error $\mathbf{E}$ of the Du Fort-Frankel scheme (54) is given by [35]

$$
\mathbf{E}=-v \frac{\Delta t^{2}}{\Delta x^{2}} \partial_{t t}^{2} \rho+O\left(\Delta x^{2}\right)
$$

As a consequence, the (first order) equivalent equation associated to the Du Fort-Frankel scheme is the telegraph equation

$$
\partial_{t} \rho=v\left(\partial_{x x}^{2} \rho-\frac{\partial_{t t}^{2} \rho}{c^{2}}\right) \quad \text { with } \quad c=\frac{\Delta x}{\Delta t}
$$

which implies that the Du Fort-Frankel scheme is consistent with the diffusion equation if and only if

$$
\Delta t=O\left(\Delta x^{\beta}\right) \quad \text { with } \quad \beta>1 .
$$

As a consequence, the LBM scheme (35)(44) and the LBM* scheme (38)(44) cannot be consistent with the convection-diffusion equation (1) when $O\left(C_{d} \Delta x\right) \geq 1$.

The consistency condition (83) limits the range of the unconditionnal stability in $L^{\infty}$ of the LBM schemes (50) and (51) obtained in the case of the diffusion equation (see Proposition 5.2; see also the discrete maximum principles obtained in §6) and which makes robust these LBM schemes. Let us note that the LBM schemes (50) and (51) are equivalent to

$$
\left\{\begin{array}{l}
g_{1, i}^{n+1}=g_{1, i+1}^{n} \\
g_{2, i}^{n+1}=g_{2, i-1}^{n}, \\
\rho_{i}^{n+1}=g_{1, i}^{n+1}+g_{2, i}^{n+1}
\end{array}\right.
$$

when $\eta:=\frac{1}{C_{d}+\frac{1}{2}}=0$ that is to say when $C_{d} \rightarrow+\infty$. This confirms the fact that the LBM schemes (50) and (51) cannot be consistent with the diffusion equation when $O\left(C_{d} \Delta x\right) \geq 1$ since (84)(a,b) are two convective schemes with $C F L=1$.

\section{LBM schemes applied to the convection equation with periodic boundary conditions}

By linking $\Delta t$ and $\Delta x$ with

$$
\Delta t:=C_{c} \frac{\Delta x}{\max _{j}\left|u\left(x_{j}\right)\right|} \quad \text { with } \quad C_{c}=O(1)
$$

( $C_{c}$ is the CFL constant) instead of (17), and by replacing $\eta$ defined with (32) with

$$
\left.\left.\hat{\eta}:=\frac{2}{C_{c}+1}=\frac{2}{\frac{\Delta t}{\Delta x} \max _{j}\left|u\left(x_{j}\right)\right|+1} \in\right] 0,2\right],
$$


we now explain that the results obtained in the case of the convection-diffusion equation (1) allow to prove without any new difficulties that the LBM schemes (35)(44) and (38)(44) solve the convection equation

$$
\left\{\begin{array}{l}
\partial_{t} \rho+\partial_{x}(u \rho)=0, \\
\rho(0, x)=\rho^{0}(x),
\end{array}\right.
$$

and that these LBM schemes are now equivalent to the finite difference type scheme

$$
\frac{\rho_{i}^{n+1}-\rho_{i}^{n-1}}{2 \Delta t}+\frac{1}{2 \Delta x}\left[u\left(x_{i+1}\right) \rho_{i+1}^{n}-u\left(x_{i-1}\right) \rho_{i-1}^{n}\right]=\frac{\max _{j}\left|u\left(x_{j}\right)\right|}{2 \Delta x}\left(\rho_{i+1}^{n}-\rho_{i}^{n+1}-\rho_{i}^{n-1}+\rho_{i-1}^{n}\right) .
$$

In particular, when $u(x)$ is a constant $u_{0}$ in (88), the previous results allow to obtain the convergence in $L^{\infty}$ and a discrete maximum principle under a classical CFL condition.

\subsection{Link with a finite difference type scheme}

The following result is a corollary of Lemma 4.1:

Lemma 8.1. In the periodic case:

i) The LBM scheme (35)(44) with $\eta$ replaced by $\hat{\eta}$ is identical to the finite difference type scheme (46)(88).

ii) The $L B M^{*}$ scheme (38)(44) with $\eta$ replaced by $\hat{\eta}$ is identical to the finite difference type scheme (47)(88).

iii) The LBM scheme (35)(44) and the LBM* scheme (38)(44) with $\eta$ replaced by $\hat{\eta}$ are identical if and only if $\alpha=\beta=\frac{1}{2}$.

Before proving a convergence result in $L^{\infty}$, we can already say that Lemma 7.1 is also verified by the LBM schemes (35)(44) and (38)(44) with $\eta$ replaced by $\hat{\eta}$, and thus by the scheme (47)(88).

Proof of Lemma 8.1: We just have to apply the proof of Proposition 4.1 with

$$
v:=\max _{j}\left|u\left(x_{j}\right)\right| \frac{\Delta x}{2} .
$$

In particular, (17) and (85) with (89) imply that $C_{c}=2 C_{d}$, which means that (32) has to be replaced by (86).

\subsection{Convergence in $L^{\infty}$ and discrete maximum principle with constant velocity}

We are now interested in the advection equation

$$
\left\{\begin{array}{l}
\partial_{t} \rho+u_{0} \partial_{x} \rho=0, \\
\rho(0, x)=\rho^{0}(x)
\end{array}\right.
$$

where $u_{0} \in \mathbb{R}$ is a constant velocity. Let us note that when $u_{0} \geq 0$, the LBM schemes (35)(44) and (38)(44) are respectively given by

$$
\left\{\begin{array}{l}
g_{1, i}^{n+1}=(\hat{\eta}-1) g_{2, i+1}^{n}, \\
g_{2, i}^{n+1}=(2-\hat{\eta}) g_{2, i-1}^{n}+g_{1, i-1}^{n}, \\
\rho_{i}^{n+1}=g_{1, i}^{n+1}+g_{1, i}^{n+1} \\
26
\end{array}\right.
$$


and

$$
\left\{\begin{array}{l}
g_{1, i}^{n+1}=(\hat{\eta}-1) g_{2, i-1}^{n}, \\
g_{2, i}^{n+1}=(2-\hat{\eta}) g_{2, i-1}^{n}+g_{1, i+1}^{n}, \\
\rho_{i}^{n+1}=g_{1, i}^{n+1}+g_{1, i}^{n+1}
\end{array}\right.
$$

(we have similar formulae when $u_{0}<0$ ). The following result is a corollary of Proposition 5.1:

Proposition 8.1. Let us suppose that the boundary conditions are periodic and that $u(x)$ is constant and equal to $u_{0}$. Then, under the condition

$$
\begin{cases}C_{c} \in[0,1], & \text { (a) } \\ \alpha \in[0,1], & \text { (b) } \\ \beta=0 \quad \text { or } \quad \beta=\min (1-\alpha, \alpha), & \text { (c) }\end{cases}
$$

we have:

i) The LBM scheme (35)(44) and the $L B M^{*}$ scheme (38)(44) with $\eta$ replaced by $\hat{\eta}$ converge in $L^{\infty}$ and verify the discrete maximum principle

$$
\forall i, \forall n \geq 0: \quad \min _{j} \rho_{j}^{0} \leq \rho_{i}^{n} \leq \max _{j} \rho_{j}^{0} .
$$

ii) The scheme (47)(88) converges in $L^{\infty}$ and verifies (94).

iii) The error of the schemes (35)(44) and (38)(44) with $\eta$ replaced by $\hat{\eta}$, and of (47)(88) is of order $\Delta x$ for any $(\alpha, \beta) \in \mathbb{R}^{2}$.

The LBM scheme (35)(44) with constant velocity $u_{0}$ and with $g_{q, i}^{0}=M_{q, i}^{0}$ (that is to say with $\alpha=\beta=1 / 2$ : see (44) and (48)) is also proposed in [18] to solve the advection equation (90). It is proved in [18] with the von Neumann stability analysis that this LBM scheme is stable in $L^{2}$ under the condition $\left|u_{0}\right| \leq 1$ (see Theorem 2 in [18]): this condition is the CFL condition (93)(a) since $\Delta t=\Delta x$ in [18] (which implies that $\left.C_{c}=\left|u_{0}\right|\right)$. At last, let us note that (86) implies that the CFL condition (93)(a) is equivalent to the condition $\hat{\eta} \in[1,2]$ (the link between $\hat{\eta}$ - noted $\omega$ in [18] -, $\Delta t$ and $\Delta x$ is not detailed in [18]).

Proof of Proposition 8.1: We just have to apply the proof of Proposition 5.1 with $v$ given by (89) and by noting that the condition (70)(a) is now given by

$$
\left.\Delta x \in] 0, \frac{\min \left(1,2 C_{d}\right)}{2 C_{d}} \Delta x\right],
$$

which is satisfied if and only if $\left.\left.C_{d} \in\right] 0,1 / 2\right]$ that is to say if and only if $\left.\left.C_{c} \in\right] 0,1\right]$ since $C_{c}=2 C_{d}$. We conclude the proof of points $i$ and $i i$ by noting that the case $C_{c}=0$ is a consequence of Lemma 7.1. At last, we prove the point $i i i$ by showing that the consistency error $\mathbf{E}$ is given by

$$
\mathbf{E}=\left|u_{0}\right| \frac{\Delta x}{2}\left(1-C_{c}^{2}\right) \partial_{x x}^{2} \rho+O\left(\Delta x^{2}\right)
$$

(see $\S 8.3$ ) and by noting that the error of the first iterate (47) is also of order $\Delta x$ for any $(\alpha, \beta) \in \mathbb{R}^{2}$ and any $C_{c} \geq 0$ 


\subsection{Consistency error with constant velocity}

When the solution $\rho(t, x)$ of the advection equation (90) is enough regular, it also satisfies

$$
\partial_{t t}^{2} \rho-\left|u_{0}\right|^{2} \partial_{x x}^{2} \rho=0 .
$$

On the other side, the consistency error of the centered discretization of $u_{0} \partial_{x} \rho$ in (88) is of order $\Delta x^{2}$. Thus, by using the consistency error (81) of the Du Fort-Frankel scheme, the relations (89) and (95), we obtain that the consistency error $\mathbf{E}$ of (88) with $u(x)=u_{0}$ is given by

$$
\begin{aligned}
\mathbf{E} & =\left|u_{0}\right| \frac{\Delta x}{2}\left[\partial_{x x}^{2} \rho-\left(\frac{\Delta t}{\Delta x}\right)^{2} \partial_{t t}^{2} \rho\right]+O\left(\Delta x^{2}\right) \\
& =\left|u_{0}\right| \frac{\Delta x}{2}\left(1-C_{c}^{2}\right) \partial_{x x}^{2} \rho+O\left(\Delta x^{2}\right)
\end{aligned}
$$

which implies that the (first order) equivalent equation associated to the scheme (88) with $u(x)=u_{0}$ is

$$
\partial_{t} \rho+u_{0} \partial_{x} \rho=\left|u_{0}\right| \frac{\Delta x}{2}\left(1-C_{c}^{2}\right) \partial_{x x}^{2} \rho .
$$

This result underlines that (88) with $u(x)=u_{0}$ is a first order scheme applied to (90), and is coherent with the CFL condition (93)(a). Let us note that by using (33) (with $\hat{\eta}$ instead of $\eta$ ) and (89), we can write that $\mathbf{E}=\left(\frac{1}{\hat{\eta}}-\frac{1}{2}\right)\left(1-C_{c}^{2}\right) \frac{\Delta x^{2}}{\Delta t} \partial_{x x}^{2} \rho+O\left(\Delta x^{2}\right)$. This formula is exactly the one obtained in [18] (see (23)-(25) in [18] with $\omega:=\hat{\eta}$ and $|a|=C_{c}$; we recall that $\Delta t=\Delta x$ in [18], which implies in particular that the velocity $\left|u_{0}\right|$ noted $|a|$ in [18] is equal to our CFL constant $C_{c}$ ).

\subsection{Exact solution with constant velocity}

The equivalent equation (96) suggests that when $C_{c}=1$ (i.e. $\left.\Delta t=\Delta x /\left|u_{0}\right|\right)$, the scheme (88) (and the LBM schemes) with $u(x)=u_{0}$ solves exactly the advection equation (90). Indeed, we have the following property:

Property 8.1. Let us suppose that the boundary conditions are periodic and that $u(x)$ is constant and equal to $u_{0}$. Then, when $C_{c}=1$ :

i) For any $(\alpha, \beta) \in \mathbb{R}^{2}$, the LBM scheme (35)(44) with $\eta$ replaced by $\hat{\eta}$ and the finite difference type scheme (46)(88) verify

$$
\left\{\begin{array}{lll}
\rho_{i}^{n+1}=\rho_{i-1}^{n} & \text { when } & u_{0}>0, \\
\rho_{i}^{n+1}=\rho_{i+1}^{n} & \text { when } & u_{0}<0 .
\end{array}\right.
$$

ii) When $\alpha=\beta=\frac{1}{2}$, the LBM* scheme (38)(44) with $\eta$ replaced by $\hat{\eta}$ and the finite difference type scheme (47)(88) verify (97).

Let us recall that when $C_{c}=1$, (97) is also satisfied by the classical upwind scheme applied to (90).

Proof of Property 8.1: Let us suppose that $C_{c}=1$, that is to say $\hat{\eta}=1$. We also suppose that $u_{0} \geq 0$ (the proof is identical when $u_{0}<0$ ). 
- Proof of point $i$ : We deduce from (91) that

$$
\left\{\begin{array}{l}
g_{1, i}^{n+1}=0 \\
g_{2, i}^{n+1}=g_{2, i-1}^{n}+g_{1, i-1}^{n} \\
\rho_{i}^{n+1}=g_{1, i}^{n+1}+g_{2, i}^{n+1}
\end{array}\right.
$$

which implies that $\rho_{i}^{n+1}=g_{2, i-1}^{n}+g_{1, i-1}^{n}=\rho_{i-1}^{n}$. We obtain the result for the scheme (46)(88) by using the point $i$ of Lemma 8.1.

- Proof of point ii: This is a consequence of points $i i$ and iii of Lemma 8.1.

\section{Probabilistic interpretation of the LBM schemes for the diffusion equation}

We now propose two Monte-Carlo algorithms deduced from the LBM scheme (50) and from the LBM* scheme (51) in the case of periodic boundary conditions. To obtain these Monte-Carlo algorithms, we define

$$
g^{n}(x, v)=w \sum_{k=1}^{\mathcal{K}} \delta\left(x-X_{k}^{n}\right) \cdot \delta\left(v-V_{k}^{n}\right)
$$

where the numerical particles $k \in\{1, \ldots, \mathcal{K}\}$ are characterized by the position $X_{k}^{n} \in\left\{x_{1}, \ldots, x_{N}\right\}\left(x_{i} \in \Omega\right)$, the velocity $V_{k}^{n} \in\left\{-\frac{\Delta x}{\Delta t}, \frac{\Delta x}{\Delta t}\right\}$ and the weight $w \in \mathbb{R}$ supposed to be constant, $\left(\left\{X_{k}^{n}\right\}_{k},\left\{V_{k}^{n}\right\}_{k}\right)$ being a random process which will be defined below and $\delta(\cdot)$ being the Dirac distribution. The weak formulation of $g^{n}(x, v)$ is given by

$$
\int_{\Omega \times \mathbb{R}} g^{n}(x, v) \varphi(x, v) d x d v=\sum_{i}\left[g_{1, i}^{n} \varphi\left(x_{i},-\frac{\Delta x}{\Delta t}\right)+g_{2, i}^{n} \varphi\left(x_{i}, \frac{\Delta x}{\Delta t}\right)\right] \Delta x
$$

with

$$
\left\{\begin{array}{l}
g_{1, i}^{n}=\frac{w}{\Delta x} \operatorname{card}\left(\left\{k / X_{k}^{n}=x_{i} \text { and } V_{k}^{n}=-\frac{\Delta x}{\Delta t}\right\}\right), \\
g_{2, i}^{n}=\frac{w}{\Delta x} \operatorname{card}\left(\left\{k / X_{k}^{n}=x_{i} \text { and } V_{k}^{n}=\frac{\Delta x}{\Delta t}\right\}\right) .
\end{array}\right.
$$

The density $\rho_{i}^{n}=g_{1, i}^{n}+g_{2, i}^{n}$ is thus given by

$$
\rho_{i}^{n}=\frac{w}{\Delta x} \operatorname{card}\left(\left\{k / X_{k}^{n}=x_{i}\right\}\right) .
$$

Moreover, we initialize the particles with the random process

$$
\left\{\begin{aligned}
X_{k}^{0} & =x_{i} \text { with the discrete probability } P\left(X_{k}^{0}=x_{i}\right)=\frac{\rho\left(0, x_{i}\right)-\min _{l} \rho\left(0, x_{l}\right)}{\sum_{j}\left[\rho\left(0, x_{j}\right)-\min _{l} \rho\left(0, x_{l}\right)\right]}, \\
V_{k}^{0} & =\frac{\Delta x}{\Delta t} \text { with the probability } \alpha \\
& =-\frac{\Delta x}{\Delta t} \text { with the probability } 1-\alpha
\end{aligned}\right.
$$


which is a consequence of the initial condition (52), $\rho(0, x)$ being the initial condition (due to the random nature of (98), we only have $\rho_{i}^{0} \simeq \rho\left(0, x_{i}\right)$ ). The random process (98) implies that we have to impose

$$
\alpha \in[0,1] .
$$

At last, the weight $w$ is defined with

$$
w=\frac{\sum_{i} \rho_{i}^{0}}{\mathcal{K}} \Delta x
$$

which comes from the conservation constraint

$$
\int_{\Omega \times \mathbb{R}} g^{n}(x, v) d x d v=\sum_{i} \rho_{i}^{0} \Delta x
$$

\subsection{Monte-Carlo algorithm for the LBM scheme}

The LBM scheme (50) can be written with the following splitting collision-transport:

\section{Collision:}

$$
\left\{\begin{array}{l}
g_{1, i}^{*}=g_{1, i}^{n}\left(1-\frac{\eta}{2}\right)+g_{2, i}^{n} \frac{\eta}{2} \\
g_{2, i}^{*}=g_{2, i}^{n}\left(1-\frac{\eta}{2}\right)+g_{1, i}^{n} \frac{\eta}{2}
\end{array}\right.
$$

Transport:

$$
\left\{\begin{array}{l}
g_{1, i}^{n+1}=g_{1, i+1}^{*}, \\
g_{2, i}^{n+1}=g_{2, i-1}^{*}
\end{array}\right.
$$

This incites us to approach (99)(100) with the random algorithm

$$
\left\{\begin{aligned}
V_{k}^{n+1} & =V_{k}^{n} \text { with the probability } 1-\frac{\eta}{2} \\
& =-V_{k}^{n} \text { with the probability } \frac{\eta}{2} \\
X_{k}^{n+1} & =X_{k}^{n}+\Delta t V_{k}^{n+1}
\end{aligned}\right.
$$

where

$$
\eta=\frac{1}{C_{d}+\frac{1}{2}}
$$

We recall that $\Delta t:=C_{d} \frac{\Delta x^{2}}{v}$ with $C_{d} \geq 0$. Let us underline that since $\left.\left.\eta \in\right] 0,2\right]$, the random process (101) is always defined. Moreover, it describes a 1D Brownian motion. Let us note that a similar Monte-Carlo algorithm has been proposed in [41] for the telegraph equation (82). 


\subsection{Monte-Carlo algorithm for the $\mathrm{LBM}^{*}$ scheme}

In the same way, the LBM* scheme (51) can be written with the following splitting transport-collision:

Transport:

$$
\left\{\begin{array}{l}
g_{1, i}^{*}=g_{1, i+1}^{n}, \\
g_{2, i}^{*}=g_{2, i-1}^{n} .
\end{array}\right.
$$

Collision:

$$
\left\{\begin{array}{l}
g_{1, i}^{n+1}=g_{1, i}^{*}\left(1-\frac{\eta}{2}\right)+g_{2, i}^{*} \frac{\eta}{2}, \\
g_{2, i}^{n+1}=g_{2, i}^{*}\left(1-\frac{\eta}{2}\right)+g_{1, i}^{*} \frac{\eta}{2} .
\end{array}\right.
$$

This incites us to approach (102)(103) with the random algorithm

$$
\left\{\begin{aligned}
X_{k}^{n+1} & =X_{k}^{n}+\Delta t V_{k}^{n}, \\
V_{k}^{n+1} & =V_{k}^{n} \text { with the probability } 1-\frac{\eta}{2} \\
& =-V_{k}^{n} \text { with the probability } \frac{\eta}{2} .
\end{aligned}\right.
$$

We show below the relation between the Monte-Carlo algorithms (98)(101) and (98)(104).

\subsection{Some properties of the Monte-Carlo algorithms}

We present some properties satisfied by the Monte-Carlo algorithms (98)(101) and (98)(104).

\subsubsection{Discrete maximum principle}

We have the following result:

Lemma 9.1. For any $C_{d} \geq 0$, the Monte-Carlo algorithms (98)(101) and (98)(104) verify the discrete maximum principle

$$
\forall i, \forall n \geq 0: \quad \min _{j} \rho_{j}^{0} \leq \rho_{i}^{n} \leq \max _{j} \rho_{j}^{0} .
$$

We recall that the Monte-Carlo algorithms (98)(101) and (98)(104) are defined when $\alpha \in[0,1]$. Lemma 9.1 is coherent with the fact that the LBM schemes (50)(52) and (51)(52) with periodic boundary conditions verify the discrete maximum principle (105) when $\alpha \in[0,1]$ (see point $i$ of Proposition 6.1).

Proof of Lemma 9.1: We have $\rho_{i}^{n}=N_{i}^{n} \frac{w}{\Delta x}$ where $N_{i}^{n}$ is the number of particles in the cell $i$ at the time $t^{n}$. Since $w=\frac{\sum_{j} \rho_{j}^{0}}{\mathcal{K}} \Delta x$, we obtain $\rho_{i}^{n}=\frac{N_{i}^{n}}{\mathcal{K}} \sum_{j} \rho_{j}^{0}$ that is to say

$$
\rho_{i}^{n}=\frac{N_{i}^{n}}{\sum_{i} N_{i}^{n}} \sum_{j} \rho_{j}^{0}
$$

since $\mathcal{K}=\sum_{i} N_{i}^{n}$. Thus, $\rho_{i}^{n}$ is a convex combination of $\left\{\rho_{j}^{0}\right\}_{j}$ which allows to conclude. 


\subsubsection{Link between the Monte-Carlo algorithms}

We can remark that the Monte-Carlo algorithms (98)(101) and (98)(104) are similar. More precisely, the LBM scheme (98)(101) can be rewritten with

$$
\left\{\begin{aligned}
X_{k}^{0} & =x_{i} \text { with the discrete probability } P\left(X_{k}^{0}=x_{i}\right)=\frac{\rho\left(0, x_{i}\right)-\min _{l} \rho\left(0, x_{l}\right)}{\sum_{j}\left[\rho\left(0, x_{j}\right)-\min _{l} \rho\left(0, x_{l}\right)\right]}, \\
\bar{V}_{k}^{0} & =\frac{\Delta x}{\Delta t} \text { with the probability } \alpha, \\
& =-\frac{\Delta x}{\Delta t} \text { with the probability } 1-\alpha, \\
V_{k}^{0} & =\bar{V}_{k}^{0} \text { with the probability } 1-\frac{\eta}{2} \\
& =-\bar{V}_{k}^{0} \text { with the probability } \frac{\eta}{2}
\end{aligned}\right.
$$

and

But, the random process

$$
\left\{\begin{aligned}
X_{k}^{n+1} & =X_{k}^{n}+\Delta t V_{k}^{n}, \\
V_{k}^{n+1} & =V_{k}^{n} \text { with the probability } 1-\frac{\eta}{2} \\
& =-V_{k}^{n} \text { with the probability } \frac{\eta}{2}
\end{aligned}\right.
$$

is equivalent to the random process

$$
\left\{\begin{aligned}
\bar{V}_{k}^{0} & =\frac{\Delta x}{\Delta t} \text { with the probability } \alpha, \\
& =-\frac{\Delta x}{\Delta t} \text { with the probability } 1-\alpha, \\
V_{k}^{0} & =\bar{V}_{k}^{0} \text { with the probability } 1-\frac{\eta}{2} \\
& =-\bar{V}_{k}^{0} \text { with the probability } \frac{\eta}{2}
\end{aligned}\right.
$$

$$
\left\{\begin{aligned}
V_{k}^{0} & =\frac{\Delta x}{\Delta t} \text { with the probability } \xi \\
& =-\frac{\Delta x}{\Delta t} \text { with the probability } 1-\xi
\end{aligned}\right.
$$

with $\xi=\alpha\left(1-\frac{\eta}{2}\right)+(1-\alpha) \frac{\eta}{2}$ that is to say with

$$
\xi=\frac{\eta}{2}+\alpha(1-\eta)
$$

which belongs to $[0,1]$ since $\alpha \in[0,1]$ and $\eta \in] 0,2]$. To summarize, the LBM scheme (98)(101) is equivalent to the $\mathrm{LBM}^{*}$ scheme (98)(104) by replacing $\alpha$ with $\xi$ in (98). This result is coherent with the fact that the LBM schemes (50)(52) and (51)(52) are equivalent to the Du Fort-Frankel scheme whose the first iterate is respectively given by

$$
\rho_{i}^{n=1}:=\xi \rho_{i-1}^{0}+(1-\xi) \rho_{i+1}^{0}
$$

and by

$$
\rho_{i}^{n=1}:=\alpha \rho_{i-1}^{0}+(1-\alpha) \rho_{i+1}^{0}
$$

(see Lemma 4.1). 


\subsubsection{Other properties}

Let us suppose that $C_{d}=0$. Thus, we have $\eta=2$ which implies that the Monte-Carlo algorithms (101) and (104) are deterministic and verify $V_{k}^{n+1}=-V_{k}^{n}$. As a consequence, we obtain

$$
\forall i, \forall n \geq 0: \quad \rho_{i}^{n+2}=\rho_{i}^{n}
$$

which corresponds to Lemma 7.2.

We now suppose that $C_{d} \rightarrow+\infty$. In that case, we have $\eta=0$. Again, the Monte-Carlo algorithms (101) and (104) are deterministic but verify now $V_{k}^{n+1}=V_{k}^{n}$. Thus, the particles move to the left or to the right without changing their initial velocity: in that case, the Monte-Carlo algorithms (98)(101) and (98)(104) do not converge toward the solution of the diffusion equation. This situation is related to the consistency condition of the LBM schemes described in $\$ 7.2$.

At last, let us suppose that $C_{d}=\frac{1}{2}$. In that case, we easily verify that the Du Fort-Frankel scheme is equivalent to the classical three points finite difference scheme for the diffusion equation (see (155) with $\eta=1$ and $u(x)=0$ ). On the other side, the Monte-Carlo algorithms (101) and (104) describe a discrete brownian motion which is the Monte-Carlo version of the three points scheme for the diffusion equation. These remarks are coherent with the fact that the LBM schemes (50) and (51) are equivalent to the Du Fort-Frankel scheme and, thus, are equivalent to the three points scheme when $C_{d}=\frac{1}{2}$.

\subsection{Convergence of the Monte-Carlo algorithms}

It remains to prove that the Monte-Carlo algorithms (98)(101) and (98)(104) converge to the solution of the diffusion equation with probabilistic tools, which would be a probabilistic version of Proposition 5.2 in the periodic case. More generally, it would be interesting to extend the Monte-Carlo algorithms (98)(101) and (98)(104) to the Neumann and Dirichlet cases, and to verify the convergence of these schemes with probabilistic tools. Moreover, it would be also interesting to prove the convergence of the LBM schemes (50) and (51) with modified Dirichlet boundary conditions (see Conjecture 6.1) by using a probabilistic approach. At last, the case of the LBM schemes (35) and (38) with $u(x) \neq 0$ should be also studied with a probabilistic approach. A starting point could be [41, 42].

\section{Numerical results}

We now present numerical results which illustrate some of the results presented before in the case of the diffusion equation. In the following test-cases, we choose $x_{\max }=-x_{\min }=10$ and $v=1$. Moreover, $N$ is the number of cells: in the periodic or Neumann cases, we have $N \Delta x=x_{\max }-x_{\min }$ and in the Dirichlet case, we have $(N+1) \Delta x=x_{\max }-x_{\min }$. At last, we recall that $\Delta t=C_{d} \frac{\Delta x^{2}}{v}$ and that $\eta=\frac{1}{C_{d}+\frac{1}{2}}$.

10.1. LBM $^{*}$ scheme

- Test-case 1: Discrete maximum principle with Dirichlet boundary conditions

We test the the $\mathrm{LBM}^{*}$ scheme (51)(52) with $\alpha=\frac{1}{2}$ when we apply the Dirichlet boundary conditions $\rho\left(t, x_{\min }\right)=\rho_{x_{\min }}$ and $\rho\left(t, x_{\max }\right)=\rho_{x_{\max }}$. At the discrete level, we use the Dirichlet boundary conditions (63) 
in $x=x_{\min }$ and its extension in $x=x_{\max }$ that is to say

$$
\left\{\begin{array}{l}
g_{2, i=0}^{n+1}=\frac{\rho_{x_{\min }}}{2}+\left(\frac{\rho_{x_{\min }}}{2}-g_{1, i=1}^{n}\right)(1-\eta), \\
g_{2, i=0}^{n=0}=\frac{\rho_{x_{\min }}}{2}, \\
g_{1, i=N+1}^{n+1}=\frac{\rho_{x_{\max }}}{2}+\left(\frac{\rho_{x_{\max }}}{2}-g_{2, i=N}^{n}\right)(1-\eta), \\
g_{1, i=N+1}^{n=0}=\frac{\rho_{x_{\max }}}{2} .
\end{array}\right.
$$

We also use the modified Dirichlet boundary conditions (77) in $x=x_{\min }$ and its extension in $x=x_{\max }$ that is to say

$$
\forall n \geq 0:\left\{\begin{array}{l}
g_{2, i=0}^{n}=\frac{\rho_{x_{\min }}}{2}, \\
g_{1, i=N+1}^{n}=\frac{\rho_{x_{\max }}}{2} .
\end{array}\right.
$$

We recall that the boundary conditions (109) allow to satisfy a discrete maximum principle for any $C_{d} \geq 0$ which is not the case of (108): see Lemma 6.1 and Proposition 6.2. To illustrate this result, we choose $N=10-$ that is to say a low number of cells -, we define the following initial condition

$$
\begin{aligned}
\rho_{i}^{0} & =\frac{1}{4} \text { if } i \notin\{5,6\}, \\
& =\frac{3}{4} \text { if } i \in\{5,6\}
\end{aligned}
$$

and we choose $C_{d}=4$. Figures 1-2 show the results respectively with $\rho_{x_{\min }}=\rho_{x_{\max }}=1$ and $\rho_{x_{\min }}=\rho_{x_{\max }}=0$ when we use (108) (Figures 1-2 show also the initial condition and the stationary solution). Figures 3-4 show these results when we replace (108) by (109). We see on Figures 1-2 that the discrete maximum principle

$$
\forall i \in\{1, \ldots, N\}: \quad \min \left(\rho_{x_{\min }}, \rho_{x_{\max }}, \min _{1 \leq j \leq N} \rho_{j}^{0}\right) \leq \rho_{i}^{n} \leq \max \left(\rho_{x_{\min }}, \rho_{x_{\max }}, \max _{1 \leq j \leq N} \rho_{j}^{0}\right)
$$

is not satisfied, and that it is satisfied on Figures 3-4: this is coherent with Lemma 6.1 and Proposition 6.2.

Let us underline that although the discrete maximum principle is not satisfied on Figures 1-2, these figures show that the $\mathrm{LBM}^{*}$ scheme (51)(52)(108) is stable in $L^{\infty}$ although $C_{d}=4$, which is coherent with Proposition 5.2.

\section{- Test-case 2: Convergence order of the modified Dirichlet boundary conditions (109)}

The test-case 1 shows that to make robust the $\mathrm{LBM}^{*}$ scheme when the number of cells is low, it is better to use (109) than (108). Nevertheless, we may think that (108) is more accurate than (109). Indeed, the error of the $\mathrm{LBM}^{*}$ scheme using (108) is in $\Delta x^{2}$ since $\alpha=\frac{1}{2}$ (see Proposition 5.2) and we conjectured that the order of the $\mathrm{LBM}^{*}$ scheme using (109) is in $\Delta x$ (see Conjecture 6.1). To verify these convergence orders, we use the exact solution $\rho_{\text {exact }}(t, x)$ of the diffusion equation

$$
\rho_{\text {exact }}(t, x)=\underset{34}{\operatorname{erf}}\left[\frac{x-x_{\min }}{\sqrt{4 v(t+1)}}\right]
$$




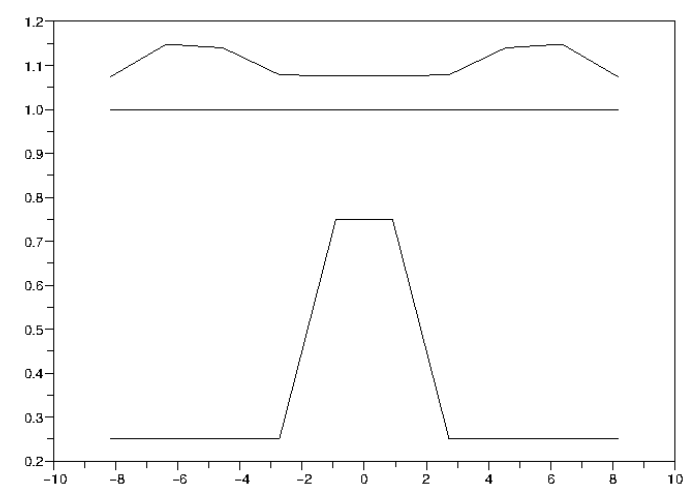

Fig. 1: LBM ${ }^{*}$ scheme with (108) when $\rho_{x_{\min }}=\rho_{x_{\max }}=1$

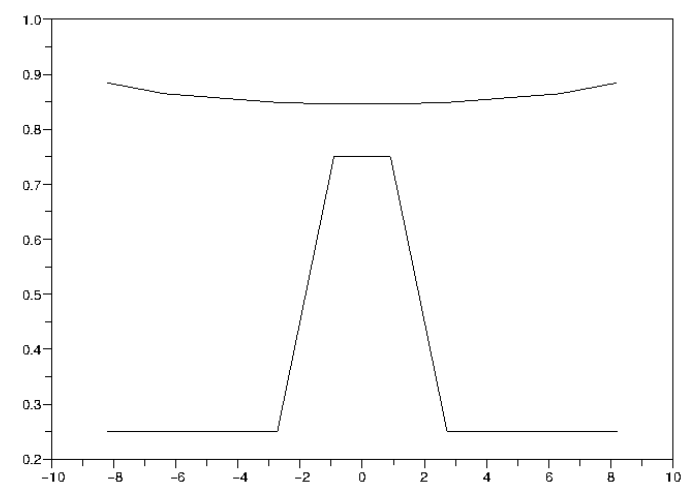

Fig. 3: LBM ${ }^{*}$ scheme with (109)

$$
\text { when } \rho_{x_{\min }}=\rho_{x_{\max }}=1
$$

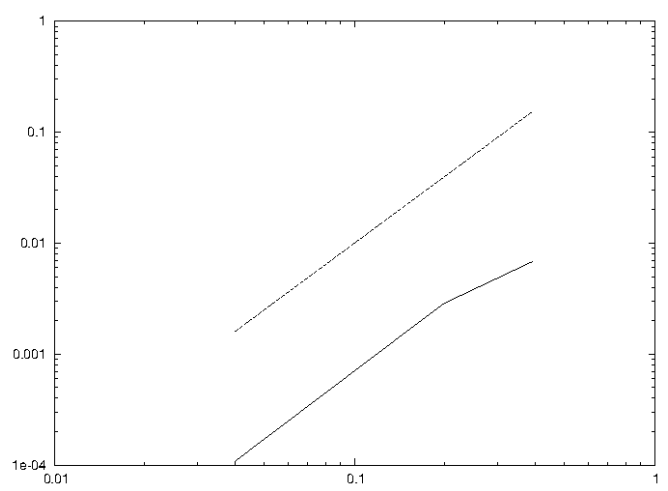

Fig. 5: LBM* scheme with (108)

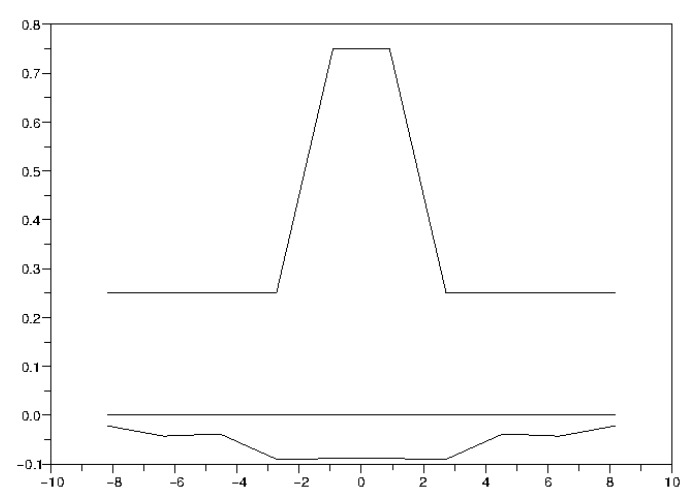

Fig. 2: LBM $^{*}$ scheme with (108) when $\rho_{x_{\min }}=\rho_{x_{\max }}=0$

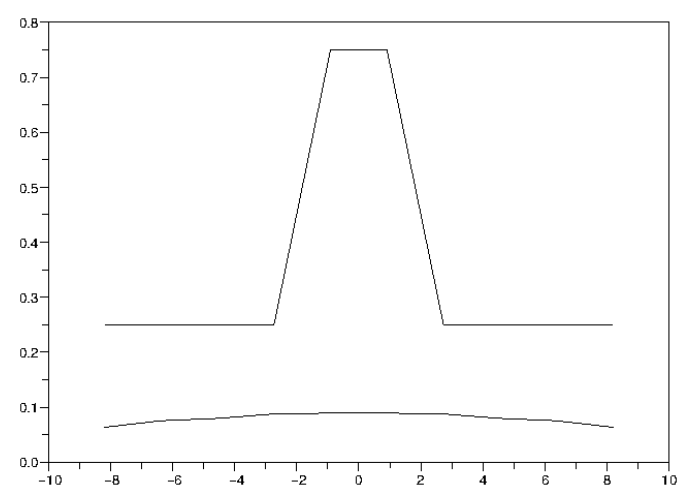

Fig. 4: LBM ${ }^{*}$ scheme with (109) when $\rho_{x_{\min }}=\rho_{x_{\max }}=0$

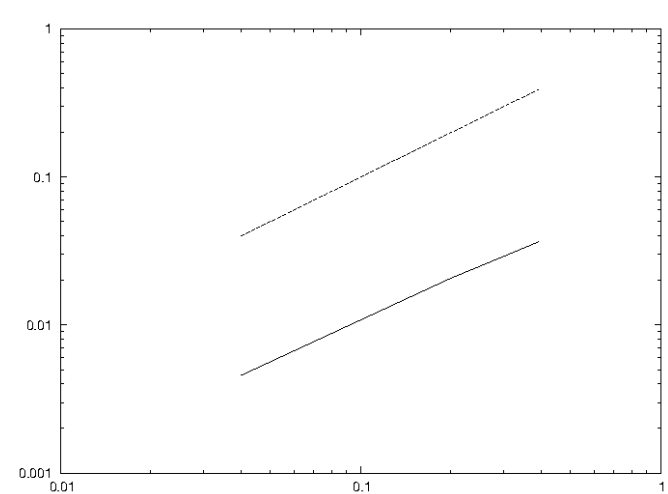

Fig. 6: LBM* scheme with (109) 
with the unstationary Dirichlet boundary conditions

$$
\left\{\begin{array}{l}
\rho_{x_{\min }}^{n}=\rho_{\text {exact }}\left(t^{n}, x_{\min }\right), \\
\rho_{x_{\max }}^{n}=\rho_{\text {exact }}\left(t^{n}, x_{\max }\right) .
\end{array}\right.
$$

Moreover, we choose $C_{d}=2, t_{\text {final }}=15$ and the number of cells $N \in\{50,100,200\}$. Figure 5 shows the function $y=x^{2}$ and the normalized $L^{2}$ error

$$
e(\Delta x):=\frac{\sqrt{\sum_{i}\left|\rho_{i}^{n}-\rho_{\text {exact }}\left(t^{n}, x_{i}\right)\right|^{2} \Delta x}}{\sqrt{\sum_{i}\left|\rho_{\text {exact }}\left(t^{n}, x_{i}\right)\right|^{2} \Delta x}}
$$

in function of $\Delta x$ in (log-log scale) when we use (108); Figure 6 shows the function $y=x$ and $e(\Delta x)$ when we use (109). These figures confirm that the error is in $\Delta x^{2}$ when we use (108) and that it is in $\Delta x$ when we use (109).

- Test-case 3: Influence of the first iterate $\rho_{i}^{n=1}$ on the Du Fort-Frankel scheme in the periodic case

We know that the LBM* scheme (51)(52) and the Du Fort-Frankel scheme (54) are equivalent when the first iterate $\rho_{i}^{n=1}$ of the Du Fort-Frankel scheme is given by

$$
\rho_{i}^{n=1}:=\alpha \rho_{i-1}^{0}+(1-\alpha) \rho_{i+1}^{0} \quad \text { where } \quad \alpha \in \mathbb{R}
$$

(see Lemma 4.1). Moreover, these schemes verify the discrete maximum principle when $\alpha \in[0,1]$ in the periodic case (see (74) in Proposition 6.1). Here, we verify the influence of the choice of (110) when $\alpha \in[0,1]$ on the Du Fort-Frankel scheme by comparing with the results obtained by replacing (110) with

$$
\rho_{i}^{n=1}:=\rho_{i}^{0} .
$$

Let us underline that the Du Fort-Frankel scheme (54) is not equivalent to the $\mathrm{LBM}^{*}$ scheme (51)(52) when the first iterate is given by (111) instead of (110). As a consequence, the discrete maximum principle may not be satisfied when we use (111). To verify this, we choose $C_{d}=4$ and a number of cells $N$ equal to 100 , and we define the initial condition with the discrete Dirac distribution

$$
\begin{aligned}
\rho_{i}^{0} & =0 \text { if } i \neq 50 \\
& =1 \text { if } i=50 .
\end{aligned}
$$

Figures 7-9 show the results after some time steps when we use the Du Fort-Frankel scheme (54) with (110) by choosing respectively $\alpha=0, \alpha=1$ and $\alpha=\frac{1}{2}$ : these figures confirm Proposition 6.1. Figure 10 shows the result when we replace (110) by (111): this figure shows that the discrete maximum principle is not satisfied by the Du Fort-Frankel scheme.

These results underline the importance of the choice of the first iterate $\rho_{i}^{n=1}$ to obtain for any $C_{d} \geq 0$ the stability in $L^{\infty}$ and a discrete maximum principle with the Du Fort-Frankel scheme. 


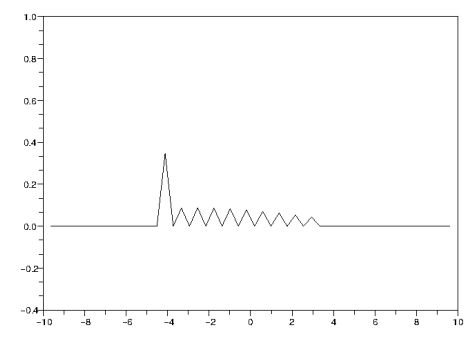

Fig. 7: Du Fort Frankel scheme with (110) and $\alpha=0$

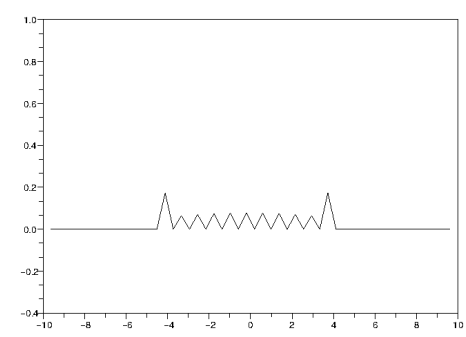

Fig. 9: Du Fort Frankel scheme with (110) and $\alpha=\frac{1}{2}$

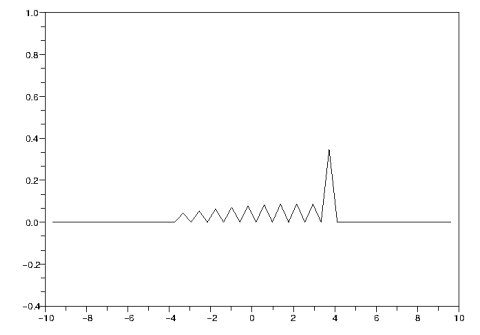

Fig. 8: Du Fort Frankel scheme with (110) and $\alpha=1$

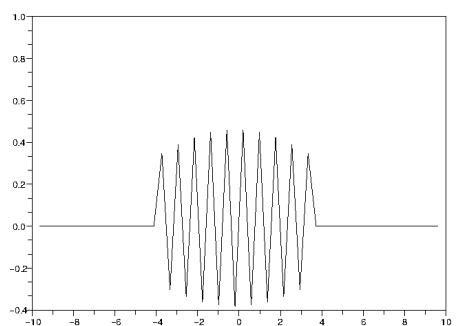

Fig. 10: Du Fort Frankel scheme with (111)

\subsection{Monte-Carlo algorithm}

We now test the Monte-Carlo algorithm (98)(104) which is the random version of the LBM$^{*}$ scheme (51)(52). The initial condition $\rho(t=0, x)$ is a gaussian function centered in $x=0$, the boundary conditions are periodic and we choose $\alpha=\frac{1}{2}$.

Figures 11-14 show the result after some time steps obtained with 100, 1000, 5000 and 10000 particles when $C_{d}=2$ and when the number of cells $N$ is equal to 100 (we also represent on these figures the initial condition and the result obtained with the $\mathrm{LBM}^{*}$ scheme). These results confirm that the Monte-Carlo algorithm converges to the $\mathrm{LBM}^{*}$ scheme when the number of particles goes to the infinity.

Figures 15 shows the result when $C_{d}=2$, when the number of cells $N$ and the number of particles $\mathcal{K}$ are equal to 1000: by comparing Figure $12(N=100$ and $\mathcal{K}=1000)$ and Figure $15(N=\mathcal{K}=1000)$, we see that $\mathcal{K}$ has to be greater than $N$ to obtain a good convergence of the Monte-Carlo algorithm. This behaviour is classical and can be justified by the fact that when $\mathcal{K}<N$, there exists at any time $t^{n}$ at least one cell where the number of particles is equal to zero.

\subsection{On the consistency condition}

Figure 16 shows the result of the test-case presented in $\S 10.2$ when $C_{d}=100, N=100$ and $\mathcal{K}=1000$ (we also represent on these figures the initial condition and the result obtained with the $\mathrm{LBM}^{*}$ scheme when $C_{d}=2$ ). Figure 17 show the result obtained with the $\mathrm{LBM}^{*}$ scheme when $C_{d}=1000$.

Figures 16 and 17 show that when $C_{d} \rightarrow+\infty$, the Monte-Carlo algorithm and the LBM* scheme do not approach the solution of the diffusion equation. This confirms the importance of the consistency condition $\Delta t=C_{d} \frac{\Delta x^{2}}{v}$ (see $\S 7.2$ and $\left.\S 9.3 .3\right)$. 


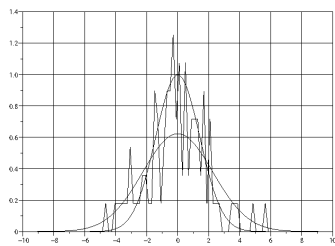

Fig. 11: 100 particles

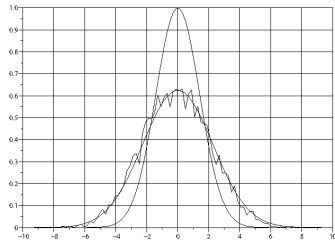

Fig. 13: 5000 particles

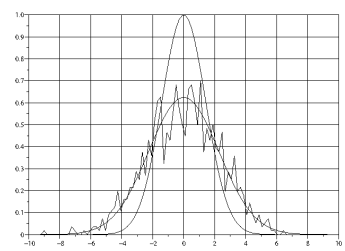

Fig. 12: 1000 particles

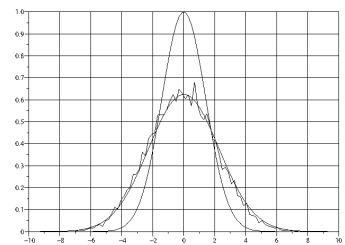

Fig. 14: 10000 particles

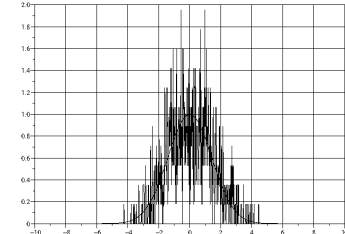

Fig. 15: 1000 cells and 1000 particles

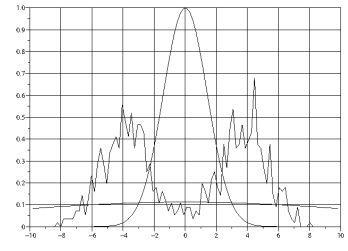

Fig. 16: 100 cells, 1000 particles and $C_{d}=100$

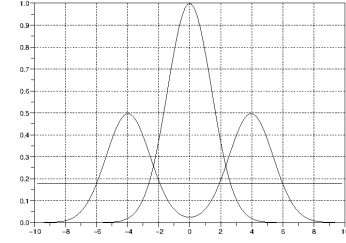

Fig. 17: LBM $^{*}$ scheme, 100 cells and $C_{d}=1000$

\section{Conclusion}

In order to construct and to justify LBM schemes for the 1D convection-diffusion equation, we have firstly studied the fluid limit of a discrete velocity kinetic system whose kinetic velocities belong to a discrete and finite set $\left\{v_{q}^{\varepsilon}\right\}_{q \in\{1,2\}}$ and whose the collision term is a BGK-type operator characterized by the collision time $\varepsilon$. This fluid limit - which is the 1D convection-diffusion equation - has been formally obtained with a Chapman-Enskog expansion and with a Hilbert expansion. The originality of this kinetic system relies on the fact that the kinetic velocity $v_{q}^{\varepsilon}$ is proportional to $1 / \sqrt{\varepsilon}$.

Then, we have constructed two LBM schemes for the 1D convection-diffusion equation by discretizing the kinetic system with a third order integration formula. With periodic boundary conditions, we have shown that theses LBM schemes are equivalent to a finite difference type scheme (initiated by a particular first iterate) which is named LFCCDF scheme. This equivalence has allowed us to obtain the convergence in $L^{\infty}$ of the LBM and LFCCDF schemes. For the diffusion equation, we have also obtained discrete maximum principles with periodic, Neumann or Dirichlet boundary conditions. These discrete maximum principles are thus satisfied by the Du Fort-Frankel (DFF) scheme since the LFCCDF scheme is a DFF scheme in the pure diffusive case. We underline that these results in $L^{\infty}$ are new for the LBM schemes but also for the LFCCDF scheme and, thus, especially for the DFF scheme which has been known for a long time to converge only in $L^{2}$ in the periodic case [35]. Moreover, by modifying the diffusion coefficient of the proposed LBM schemes, we obtained LBM schemes in the pure advective case and we again obtained stability results in $L^{\infty}$. In [18], one of these LBM schemes has been studied. Nevertheless, only stability results in $L^{2}$ in the periodic case were proved in [18]. 
In the same spirit, we have proposed two Monte-Carlo algorithms for the resolution of the diffusion equation coming from a probabilistic interpretation of the proposed LBM schemes in the periodic case. Discrete maximum principles are also obtained. These results encourage us to think that it will be possible to obtain theoretical results for the LBM schemes by also using probabilistic tools.

At last, we expect that it will be possible to analyze the properties of LBM schemes applied to more complicate equations as non-linear equations of the type $\partial_{t} \rho=\Phi\left(t, x, \rho, \partial_{x} \rho, \partial_{x x}^{2} \rho\right)$ where $\Phi(\cdot)$ is a given function by using an approach similar to the one proposed in this paper. Beyond the potential existence of links between LBM schemes, finite difference type schemes and Monte-Carlo algorithms for simple PDEs, we also expect to find links between LBM schemes applied to the incompressible Navier-Stokes system and theoretical works on discrete velocity kinetic systems [43].

Acknowledgements: We wish to thank François Drolet, David Vidal and Dominique Diplomate for helpfull discussions. We wish also to thank Christophe Le Potier who helped us to prove the equivalence between the proposed LBM schemes and the Du Fort-Frankel scheme.

\section{A. Proof of Proposition 2.1}

We now give two (formal) proofs which allow to write that the fluid limit of the kinetic system

$$
\forall q \in\{1,2\}: \quad \partial_{t} f_{q}^{\varepsilon}+v_{q}^{\varepsilon} \partial_{x} f_{q}^{\varepsilon}=\frac{1}{\varepsilon}\left(M_{q}^{\varepsilon}-f_{q}^{\varepsilon}\right) \quad \text { with } \quad v_{q}^{\varepsilon}=(-1)^{q} \sqrt{\frac{\nu}{\varepsilon}}
$$

is the equation

$$
\partial_{t} \rho^{\varepsilon}+\partial_{x}\left(u \rho^{\varepsilon}\right)=v \partial_{x x}^{2} \rho^{\varepsilon}+O(\varepsilon)
$$

and, thus, is the convection-diffusion equation (1) since we can neglect the error of the order of $\varepsilon$ in (113). One of the difficulties is linked to the fact that the kinetic velocity $v_{q}^{\varepsilon}:=(-1)^{q} \sqrt{\frac{v}{\varepsilon}}$ depends on the collision time $\varepsilon$ which is not at all classical in the framework of the kinetic theory.

The first proof is based on a Chapman-Enskog expansion; the second proof is based on a Hilbert expansion. The proof based on the Chapman-Enskog expansion is easier than the one based on the Hilbert expansion. Moreover, the Chapman-Enskog expansion allows to obtain

$$
\begin{aligned}
f_{q}^{\varepsilon}(t, x)= & \frac{\rho^{\varepsilon}}{2}\left[1+(-1)^{q} \sqrt{\varepsilon}\left(\frac{u}{\sqrt{v}}-\sqrt{v} \frac{\partial_{x} \rho^{\varepsilon}}{\rho^{\varepsilon}}\right)\right. \\
& \left.+(-1)^{q} \varepsilon^{3 / 2}\left(\frac{u}{\sqrt{v}} \cdot \frac{\partial_{x}\left(u \rho^{\varepsilon}\right)-v \partial_{x x}^{2} \rho^{\varepsilon}}{\rho^{\varepsilon}}-\sqrt{v} \frac{\partial_{x x}^{2}\left(u \rho^{\varepsilon}\right)}{\rho^{\varepsilon}}+v^{3 / 2} \frac{\partial_{x x x}^{3} \rho^{\varepsilon}}{\rho^{\varepsilon}}\right)\right]+O\left(\varepsilon^{2}\right) .
\end{aligned}
$$

With the Hilbert expansion, we only obtain

$$
f_{q}^{\varepsilon}(t, x)=\frac{\rho^{\varepsilon}}{2}\left[1+(-1)^{q} \sqrt{\varepsilon}\left(\frac{u}{\sqrt{v}}-\sqrt{v} \frac{\partial_{x} \rho^{\varepsilon}}{\rho^{\varepsilon}}\right)\right]+O\left(\varepsilon^{3 / 2}\right)
$$

which is less accurate than (114). 
The fact that the Chapman-Enskog approach is easier than the Hilbert approach is classical in the kinetic theory. In fact, the compressible Navier-Stokes system - which is the fluid limit of the classical Boltzmann equation - is obtained with a Chapman-Enskog expansion and not with a Hilbert expansion which is too complicate to give the result. Here, it is possible to obtain the fluid limit with a Hilbert expansion because the kinetic velocity set is a discrete and finite set, which implies that the linear operators are simple $2 \times 2$ matrix. Moreover, it seems to us that the Hilbert expansion is more adapted than the Chapman-Enskog expansion to clearly justify the fluid limit (113) of the kinetic system (112) because the Hilbert approach is based on a sequence of PDEs that we can study a posteriori (we do not try to do such theoretical study in the present paper). At last, the Hilbert expansion can also be seen as a (formal) justification of the ChapmanEnskog expansion since both expansions give the same result. That is why we also write the proof based on the Hilbert expansion.

At last, let us note that in the following analysis, we forget any possible influence of boundary conditions on $\partial \Omega$ : in other words, we suppose that $\Omega \subset \mathbb{R}$ is periodic. Any analysis of the influence of non-periodic boundary conditions on $\partial \Omega$ on the fluid limit of (112) is really complicate because of possible Knudsen layers in the vicinity of $\partial \Omega$ where the distribution $f_{q}^{\varepsilon}$ is not close to the maxwellian $M_{q}^{\varepsilon}$ even when $\varepsilon \ll 1$. As a consequence, we can only expect that the fluid limit (113) is valid far from the boundary $\partial \Omega$ when the boundary conditions are not periodic.

\section{A.1. Proof based on a Chapman-Enskog expansion}

Let us suppose that the solution $f_{q}^{\varepsilon}$ of (112) can be expanded with the Chapman-Enskog expansion

$$
f_{q}^{\varepsilon}=M_{q}^{\varepsilon} \cdot\left(1+\sqrt{\varepsilon} g_{1, q}^{\varepsilon}+\varepsilon g_{2, q}^{\varepsilon}+\varepsilon^{3 / 2} g_{3, q}^{\varepsilon}\right)+O\left(\varepsilon^{2}\right)
$$

under the constraints

$$
\sum_{q \in\{1,2\}} M_{q}^{\varepsilon}\left(g_{1, q}^{\varepsilon}+\sqrt{\varepsilon} g_{2, q}^{\varepsilon}\right)=0
$$

and

$$
\sum_{q \in\{1,2\}} M_{q}^{\varepsilon} g_{3, q}^{\varepsilon}=0
$$

where $g_{k, q}^{\varepsilon}$ is supposed to be of order one. We recall that the maxwellian $M_{q}^{\varepsilon}$ is given by

$$
M_{q}^{\varepsilon}:=\frac{\rho^{\varepsilon}}{2}\left(1+\frac{u}{v_{q}^{\varepsilon}}\right)=\frac{\rho^{\varepsilon}}{2}\left[1+(-1)^{q} \sqrt{\frac{\varepsilon}{v}} \cdot u\right]
$$

where $\rho^{\varepsilon}:=f_{1}^{\varepsilon}+f_{2}^{\varepsilon}$ and verifies

$$
\sum_{q \in\{1,2\}}\left(\begin{array}{c}
1 \\
v_{q}^{\varepsilon}
\end{array}\right) M_{q}^{\varepsilon}=\left(\begin{array}{c}
\rho^{\varepsilon} \\
\rho^{\varepsilon} u
\end{array}\right) .
$$

It is important to note that the constraint (117) is not classical in the framework of Chapman-Enskog expansions. Indeed, we should a priori impose

$$
\sum_{q \in\{1,2\}} M_{q}^{\varepsilon} g_{1, q}^{\varepsilon}=0 \quad \text { and } \quad \sum_{q \in\{1,2\}} M_{q}^{\varepsilon} g_{2}^{\varepsilon}=0 .
$$

Here, we replace (120) by (117) because the set of kinetic velocities $\left\{v_{q}^{\varepsilon}\right\}_{q \in\{1,2\}}$ depends on $\sqrt{\varepsilon}$ which implies that $M_{q}^{\varepsilon} g_{1, q}^{\varepsilon}$ has a term of order $\sqrt{\varepsilon}$ (and, thus, of order $\left.\sqrt{\varepsilon} g_{2, q}^{\varepsilon}\right)$ since $g_{1, q}^{\varepsilon}\left(\right.$ and $g_{2, q}^{\varepsilon}$ ) is of order one.

By injecting expansion (116) into (112), we obtain: 
- Order $\sqrt{\varepsilon}^{-1}$ : We obtain the equality

$$
M_{q}^{\varepsilon} g_{1, q}^{\varepsilon}=-\sqrt{\varepsilon} v_{q}^{\varepsilon} \partial_{x} M_{q}^{\varepsilon}=-(-1)^{q} \sqrt{v} \partial_{x} M_{q}^{\varepsilon}
$$

that is to say

$$
M_{q}^{\varepsilon} g_{1, q}^{\varepsilon}=-(-1)^{q} \sqrt{v} \frac{\partial_{x} \rho^{\varepsilon}}{2}-\sqrt{\varepsilon} \frac{\partial_{x}\left(u \rho^{\varepsilon}\right)}{2}
$$

since $M_{q}^{\varepsilon}=\frac{\rho^{\varepsilon}}{2}\left[1+(-1)^{q} \sqrt{\frac{\varepsilon}{v}} \cdot u\right]$.

- Order $\sqrt{\varepsilon}^{0}$ : We obtain the equality

$$
M_{q}^{\varepsilon} g_{2, q}^{\varepsilon}=-\left[\partial_{t} M_{q}^{\varepsilon}+\sqrt{\varepsilon} v_{q}^{\varepsilon} \partial_{x}\left(M_{q}^{\varepsilon} g_{1, q}^{\varepsilon}\right)\right] .
$$

- Order $\sqrt{\varepsilon}$ : We obtain the equality

$$
M_{q}^{\varepsilon} g_{3, q}^{\varepsilon}=-\left[\partial_{t}\left(M_{q}^{\varepsilon} g_{1, q}^{\varepsilon}\right)+\sqrt{\varepsilon} v_{q}^{\varepsilon} \partial_{x}\left(M_{q}^{\varepsilon} g_{2, q}^{\varepsilon}\right)\right] .
$$

Moreover, by summing (112) over the set $\left\{v_{q}^{\varepsilon}\right\}_{q \in\{1,2\}}$ and by injecting the expansion (116), we obtain

$$
\begin{aligned}
\partial_{t} \rho^{\varepsilon}+\partial_{x}\left(u \rho^{\varepsilon}\right)= & -\partial_{x}\left[\sum_{q \in\{1,2\}}\left(v_{q}^{\varepsilon}-u\right) f_{q}^{\varepsilon}\right] \quad\left(\text { since } \sum_{q \in\{1,2\}} u f_{q}^{\varepsilon}=u \rho^{\varepsilon} \text { and } \sum_{q \in\{1,2\}} \frac{1}{\varepsilon}\left(M_{q}^{\varepsilon}-f_{q}^{\varepsilon}\right)=0\right) \\
= & -\partial_{x}\left[\sum_{q \in\{1,2\}}\left(v_{q}^{\varepsilon}-u\right) M_{q}^{\varepsilon}\right]-\sqrt{\varepsilon} \partial_{x}\left[\sum_{q \in\{1,2\}}\left(v_{q}^{\varepsilon}-u\right) M_{q}^{\varepsilon} g_{1, q}^{\varepsilon}\right]-\varepsilon \partial_{x}\left[\sum_{q \in\{1,2\}}\left(v_{q}^{\varepsilon}-u\right) M_{q}^{\varepsilon} g_{2, q}^{\varepsilon}\right]+O(\varepsilon) \\
= & -\partial_{x}\left[\sum_{q \in\{1,2\}}\left(v_{q}^{\varepsilon}-u\right) M_{q}^{\varepsilon}\right]-\sqrt{\varepsilon} \partial_{x}\left[\sum_{q \in\{1,2\}} v_{q}^{\varepsilon} M_{q}^{\varepsilon} g_{1, q}^{\varepsilon}\right]-\varepsilon \partial_{x}\left[\sum_{q \in\{1,2\}} v_{q}^{\varepsilon} M_{q}^{\varepsilon} g_{2, q}^{\varepsilon}\right] \\
& +\sqrt{\varepsilon} \partial_{x}\left[u \sum_{q \in\{1,2\}} M_{q}^{\varepsilon}\left(g_{1, q}^{\varepsilon}+\sqrt{\varepsilon} g_{2}^{\varepsilon}\right)\right]+O(\varepsilon) .
\end{aligned}
$$

By taking into account (119), (121) and (123), we obtain

$$
\begin{aligned}
\varepsilon \partial_{x}\left[\sum_{q \in\{1,2\}} v_{q}^{\varepsilon} M_{q}^{\varepsilon} g_{2, q}^{\varepsilon}\right] & =-\varepsilon \partial_{x}\left\{\sum_{q \in\{1,2\}} v_{q}^{\varepsilon}\left[\partial_{t} M_{q}^{\varepsilon}+\sqrt{\varepsilon} v_{q}^{\varepsilon} \partial_{x}\left(M_{q}^{\varepsilon} g_{1, q}^{\varepsilon}\right)\right]\right\} \\
& =-\varepsilon \partial_{x t}^{2}\left(u \rho^{\varepsilon}\right)+\varepsilon \partial_{x}\left[\sum_{q \in\{1,2\}} v_{q}^{\varepsilon} \sqrt{\varepsilon} v_{q}^{\varepsilon} \partial_{x}\left(\sqrt{\varepsilon} v_{q}^{\varepsilon} \partial_{x} M_{q}^{\varepsilon}\right)\right] \\
& =-\varepsilon \partial_{x t}^{2}\left(u \rho^{\varepsilon}\right)+\varepsilon v \partial_{x x x}^{3}\left(u \rho^{\varepsilon}\right)=O(\varepsilon) .
\end{aligned}
$$


Thus, by also taking into account (117), we obtain

$$
\begin{aligned}
\partial_{t} \rho^{\varepsilon}+\partial_{x}\left(u \rho^{\varepsilon}\right) & =-\sqrt{\varepsilon} \partial_{x}\left[\sum_{q \in\{1,2\}} v_{q}^{\varepsilon} M_{q}^{\varepsilon} g_{1, q}^{\varepsilon}\right]+O(\varepsilon)=\varepsilon \partial_{x}\left[\sum_{q \in\{1,2\}}\left(v_{q}^{\varepsilon}\right)^{2} \partial_{x} M_{q}^{\varepsilon}\right]+O(\varepsilon) \\
& =v \partial_{x x}^{2}\left(\sum_{q \in\{1,2\}} M_{q}^{\varepsilon}\right)+O(\varepsilon)=v \partial_{x x}^{2} \rho^{\varepsilon}+O(\varepsilon)
\end{aligned}
$$

which gives (113) that is to say

$$
\partial_{t} \rho^{\varepsilon}+\partial_{x}\left(u \rho^{\varepsilon}\right)=v \partial_{x x}^{2} \rho^{\varepsilon}+O(\varepsilon) .
$$

We deduce from (122), (123) and (126) that

$$
\begin{aligned}
M_{q}^{\varepsilon} g_{2, q}^{\varepsilon} & =-\partial_{t} M_{q}^{\varepsilon}+v \partial_{x x}^{2} M_{q}^{\varepsilon} \\
& =-\frac{\partial_{t} \rho^{\varepsilon}}{2}-(-1)^{q} \sqrt{\frac{\varepsilon}{v}} \cdot \frac{\partial_{t}\left(u \rho^{\varepsilon}\right)}{2}+v \frac{\partial_{x x}^{2} \rho^{\varepsilon}}{2}+(-1)^{q} \sqrt{\varepsilon v} \cdot \frac{\partial_{x x}^{2}\left(u \rho^{\varepsilon}\right)}{2} \\
& =\frac{\partial_{x}\left(u \rho^{\varepsilon}\right)}{2}+(-1)^{q} \sqrt{\frac{\varepsilon}{v}} u \cdot \frac{\partial_{x}\left(u \rho^{\varepsilon}\right)-v \partial_{x x}^{2} \rho^{\varepsilon}}{2}+(-1)^{q} \sqrt{\varepsilon v} \cdot \frac{\partial_{x x}^{2}\left(u \rho^{\varepsilon}\right)}{2}+O(\varepsilon)
\end{aligned}
$$

(we also use the fact that $u(x)$ does not depend on the time $t$ ). This last equality encourages us to take

$$
M_{q}^{\varepsilon} g_{2, q}^{\varepsilon}=\frac{\partial_{x}\left(u \rho^{\varepsilon}\right)}{2}+(-1)^{q} \sqrt{\frac{\varepsilon}{v}} u \cdot \frac{\partial_{x}\left(u \rho^{\varepsilon}\right)-v \partial_{x x}^{2} \rho^{\varepsilon}}{2}+(-1)^{q} \sqrt{\varepsilon v} \cdot \frac{\partial_{x x}^{2}\left(u \rho^{\varepsilon}\right)}{2}
$$

since the term of order $\varepsilon$ in (127) is a term of order $\varepsilon^{2}$ in (116) and, thus, of order $\varepsilon$ in (126). We deduce from (122) and (128) that

$$
M_{q}^{\varepsilon} \cdot\left(\sqrt{\varepsilon} g_{1, q}^{\varepsilon}+\varepsilon g_{2, q}^{\varepsilon}\right)=\frac{\rho^{\varepsilon}}{2}\left[-(-1)^{q} \sqrt{\varepsilon v} \frac{\partial_{x} \rho^{\varepsilon}}{\rho^{\varepsilon}}+(-1)^{q} \frac{\varepsilon^{3 / 2}}{\sqrt{v}} u \cdot \frac{\partial_{x}\left(u \rho^{\varepsilon}\right)-v \partial_{x x}^{2} \rho^{\varepsilon}}{\rho^{\varepsilon}}+(-1)^{q} \varepsilon^{3 / 2} \sqrt{v} \cdot \frac{\partial_{x x}^{2}\left(u \rho^{\varepsilon}\right)}{\rho^{\varepsilon}}\right]
$$

which verifies the constraint (117). We deduce from (122), (124) and (128) that

$$
M_{q}^{\varepsilon} g_{3, q}^{\varepsilon}=(-1)^{q} \sqrt{v} \frac{\partial_{x x}^{2} \rho^{\varepsilon}}{2}-(-1)^{q} \sqrt{v} \frac{\partial_{x x}^{2}\left(u \rho^{\varepsilon}\right)}{2}+O(\sqrt{\varepsilon})
$$

which allows to obtain

$$
\begin{aligned}
M_{q}^{\varepsilon} g_{3, q}^{\varepsilon} & =(-1)^{q} \sqrt{v} \frac{v \partial_{x x x}^{3} \rho^{\varepsilon}-\partial_{x x}^{2}\left(u \rho^{\varepsilon}\right)}{2}-(-1)^{q} \sqrt{v} \frac{\partial_{x x}^{2}\left(u \rho^{\varepsilon}\right)}{2}+O(\sqrt{\varepsilon}) \\
& =(-1)^{q} v^{3 / 2} \frac{\partial_{x x x}^{3} \rho^{\varepsilon}}{2}-(-1)^{q} \sqrt{v} \partial_{x x}^{2}\left(u \rho^{\varepsilon}\right)+O(\sqrt{\varepsilon})
\end{aligned}
$$

by using (126). This last equality encourages us to take

$$
M_{q}^{\varepsilon} g_{3, q}^{\varepsilon}=(-1)^{q} v^{3 / 2} \frac{\partial_{x x x}^{3} \rho^{\varepsilon}}{2}-(-1)^{q} \sqrt{v} \partial_{x x}^{2}\left(u \rho^{\varepsilon}\right)
$$


since the term of order $\sqrt{\varepsilon}$ in (129) is a term of order $\varepsilon^{2}$ in (116) and, thus, of order $\varepsilon$ in (126). Let us note that (130) verifies the constraint (118). Thus, by taking into account (126), we obtain

$$
\begin{aligned}
M_{q}^{\varepsilon} \cdot\left(\sqrt{\varepsilon} g_{1, q}^{\varepsilon}+\varepsilon g_{2, q}^{\varepsilon}+\varepsilon^{3 / 2} g_{3, q}^{\varepsilon}\right)= & \frac{\rho^{\varepsilon}}{2}\left[1+(-1)^{q} \sqrt{\varepsilon}\left(\frac{u}{\sqrt{v}}-\sqrt{v} \frac{\partial_{x} \rho^{\varepsilon}}{\rho^{\varepsilon}}\right)\right. \\
& +(-1)^{q} \frac{\varepsilon^{3 / 2}}{\sqrt{v}} u \cdot \frac{\partial_{x}\left(u \rho^{\varepsilon}\right)-v \partial_{x x}^{2} \rho^{\varepsilon}}{\rho^{\varepsilon}}+(-1)^{q} \varepsilon^{3 / 2} \sqrt{v} \frac{\partial_{x x}^{2}\left(u \rho^{\varepsilon}\right)}{\rho^{\varepsilon}} \\
& \left.+(-1)^{q} \varepsilon^{3 / 2} v^{3 / 2} \frac{\partial_{x x x}^{3} \rho^{\varepsilon}}{\rho^{\varepsilon}}-2(-1)^{q} \varepsilon^{3 / 2} \sqrt{v} \frac{\partial_{x x}^{2}\left(u \rho^{\varepsilon}\right)}{\rho^{\varepsilon}}\right]
\end{aligned}
$$

that is to say

$$
\begin{aligned}
M_{q}^{\varepsilon} \cdot\left(\sqrt{\varepsilon} g_{1, q}^{\varepsilon}+\varepsilon g_{2, q}^{\varepsilon}+\varepsilon^{3 / 2} g_{3, q}^{\varepsilon}\right)= & \frac{\rho^{\varepsilon}}{2}\left[1+(-1)^{q} \sqrt{\varepsilon}\left(\frac{u}{\sqrt{v}}-\sqrt{v} \frac{\partial_{x} \rho^{\varepsilon}}{\rho^{\varepsilon}}\right)\right. \\
& \left.+(-1)^{q} \varepsilon^{3 / 2}\left(\frac{u}{\sqrt{v}} \cdot \frac{\partial_{x}\left(u \rho^{\varepsilon}\right)-v \partial_{x x}^{2} \rho^{\varepsilon}}{\rho^{\varepsilon}}-\sqrt{v} \frac{\partial_{x x}^{2}\left(u \rho^{\varepsilon}\right)}{\rho^{\varepsilon}}+v^{3 / 2} \frac{\partial_{x x x}^{3} \rho^{\varepsilon}}{\rho^{\varepsilon}}\right)\right]
\end{aligned}
$$

which gives (114) by using (116).

\section{A.2. Proof based on a Hilbert expansion}

Let us suppose that the solution $f_{q}^{\varepsilon}$ of (112) can be expanded with the Hilbert expansion

$$
f_{q}^{\varepsilon}=m_{q}^{\varepsilon} \cdot\left(g_{0, q}^{\varepsilon}+\sqrt{\varepsilon} g_{1, q}^{\varepsilon}+\sqrt{\varepsilon}^{2} g_{2, q}^{\varepsilon}+\ldots\right)
$$

where

$$
m_{q}^{\varepsilon}:=1+\frac{u}{v_{q}^{\varepsilon}}=1+(-1)^{q} \sqrt{\frac{\varepsilon}{v}} \cdot u .
$$

The density $\rho^{\varepsilon}:=f_{1}^{\varepsilon}+f_{2}^{\varepsilon}$ is given by

$$
\rho^{\varepsilon}=\rho_{0}^{\varepsilon}+\sqrt{\varepsilon} \rho_{1}^{\varepsilon}+\sqrt{\varepsilon}^{2} \rho_{2}^{\varepsilon}+\ldots
$$

with

$$
\forall n: \quad \rho_{n}^{\varepsilon}=\sum_{q \in\{1,2\}} m_{q}^{\varepsilon} g_{n, q}^{\varepsilon} .
$$

And, the maxwellian $M_{q}^{\varepsilon}$ defined with

$$
M_{q}^{\varepsilon}:=\frac{\rho^{\varepsilon}}{2}\left[1+\frac{u}{v_{q}^{\varepsilon}}\right]=\frac{\rho^{\varepsilon}}{2}\left[1+(-1)^{q} \sqrt{\frac{\varepsilon}{v}} \cdot u\right]
$$

whose density is equal to $\rho^{\varepsilon}$ is given by

$$
M_{q}^{\varepsilon}=m_{q}^{\varepsilon} \cdot\left(\rho_{0}^{\varepsilon}+\sqrt{\varepsilon} \rho_{1}^{\varepsilon}+\sqrt{\varepsilon}^{2} \rho_{2}^{\varepsilon}+\ldots\right) .
$$

In the sequel, we will prove that when the Hilbert expansion (131) is valid, the density $\rho^{\varepsilon}$ is necessarily solution of (113). Moreover, by computing $g_{0, q}^{\varepsilon}, g_{1, q}^{\varepsilon}$ and $g_{2, q}^{\varepsilon}$, we will obtain (115). 
Let us note that the difference between the Chapman-Enskog expansion (116) and the Hilbert expansion (131) can be underlined by comparing the constraints (117)(118) and the relations (132) which are not constraints, $\rho_{n}^{\varepsilon}$ being unknows which are solution of a sequence of PDEs (see below).

By injecting expansion (131) into (112), we obtain:

- $\operatorname{Order} \varepsilon^{-1}$ : We obtain the equality

$$
g_{0,1}^{\varepsilon}=g_{0,2}^{\varepsilon}=\rho_{0}^{\varepsilon}(t, x) .
$$

- $\operatorname{Order}(\sqrt{\varepsilon})^{n-1 / 2}(n \in \mathbb{N})$ : We obtain the following PDEs whose $\left\{g_{n, q}^{\varepsilon}\right\}_{n \geq 0}$ is solution:

$$
(\sqrt{\varepsilon})^{n}\left[\partial_{t}\left(m_{q}^{\varepsilon} g_{n, q}^{\varepsilon}\right)+v_{q}^{\varepsilon} \partial_{x}\left(m_{q}^{\varepsilon} g_{n, q}^{\varepsilon}\right)\right]=m_{q}^{\varepsilon}(\sqrt{\varepsilon})^{n-1}\left(\sum_{k \in\{1,2\}} m_{k}^{\varepsilon} g_{n+1, k}^{\varepsilon}-g_{n+1, q}^{\varepsilon}\right) .
$$

We recall that $O\left(\left|v_{q}^{\varepsilon}\right|\right)=1 / \sqrt{\varepsilon}$ which implies that $(\sqrt{\varepsilon})^{n} v_{q}^{\varepsilon} \partial_{x}\left(m_{q}^{\varepsilon} g_{n, q}^{\varepsilon}\right)$ and $m_{q}^{\varepsilon}(\sqrt{\varepsilon})^{n-1}\left(\sum_{k \in\{1,2\}} m_{k}^{\varepsilon} g_{n+1, k}^{\varepsilon}-g_{n+1, q}^{\varepsilon}\right)$ are formally of the same order. Moreover, we keep the unstationary term $(\sqrt{\varepsilon})^{n} \partial_{t}\left(m_{q}^{\varepsilon} g_{n, q}^{\varepsilon}\right)$ to obtain an initial value problem for $m_{q}^{\varepsilon} g_{n, q}^{\varepsilon}$. PDEs (134) can be written with the equivalent formulation

$$
\forall n \geq 0: \quad \mathbf{A}^{\varepsilon} \cdot \mathcal{G}_{n+1}=\mathcal{B}^{\varepsilon}\left(\mathcal{G}_{n}^{\varepsilon}\right)
$$

where $\mathcal{G}_{n}^{\varepsilon}=\left(g_{n, 1}^{\varepsilon}, g_{n, 2}^{\varepsilon}\right)^{T}, \mathcal{B}^{\varepsilon}=\left(b_{1}^{\varepsilon}, b_{2}^{\varepsilon}\right)^{T}$ and where

$$
\left\{\begin{array}{l}
\mathbf{A}^{\varepsilon}=\left(\begin{array}{cc}
-m_{2}^{\varepsilon} & m_{2}^{\varepsilon} \\
m_{1}^{\varepsilon} & -m_{1}^{\varepsilon}
\end{array}\right), \\
b_{q}^{\varepsilon}\left(\mathcal{G}_{n}^{\varepsilon}\right)=\frac{\sqrt{\varepsilon}}{m_{q}^{\varepsilon}} \cdot\left[\partial_{t}\left(m_{q}^{\varepsilon} g_{n, q}^{\varepsilon}\right)+v_{q}^{\varepsilon} \partial_{x}\left(m_{q}^{\varepsilon} g_{n, q}^{\varepsilon}\right)\right] .
\end{array}\right.
$$

Since the matrix $\mathbf{A}^{\varepsilon}$ is not invertible, we have to study carrefuly linear system (135). By applying the Fredholm alternative, we obtain the following result:

Lemma A.1. Let $\mathcal{G}_{-1}^{\varepsilon}=0$ and

$$
\mathcal{G}_{0}^{\varepsilon}=\left(\begin{array}{l}
1 \\
1
\end{array}\right) \rho_{0}^{\varepsilon} .
$$

Then, Equation (135) has an unique solution under the constraints

$$
\forall n \geq 0: \quad \partial_{t} \rho_{n}^{\varepsilon}+\partial_{x}\left(u \rho_{n}^{\varepsilon}\right)=\mathcal{F}^{\varepsilon}\left(\mathcal{G}_{n-1}^{\varepsilon}\right)
$$

where

$$
\forall n \geq 0: \quad \mathcal{F}^{\varepsilon}\left(\mathcal{G}_{n-1}^{\varepsilon}\right):=\partial_{x}\left[\sum_{q \in\{1,2\}}\left(v_{q}^{\varepsilon}-u\right) m_{q}^{\varepsilon} b_{q}^{\varepsilon}\left(\mathcal{G}_{n-1}^{\varepsilon}\right)\right] .
$$

Moreover, $\left\{\mathcal{G}_{n}^{\mathcal{E}}\right\}_{n \geq 1}$ is given by the recurrence relation

$$
\forall n \geq 1: \quad \mathcal{G}_{n}^{\varepsilon}=-\mathcal{B}^{\varepsilon}\left(\mathcal{G}_{n-1}^{\varepsilon}\right)+\rho_{n}^{\varepsilon}\left(\begin{array}{l}
1 \\
1
\end{array}\right) .
$$


Thus, the construction process to obtain $\left\{\mathcal{G}_{n}^{\varepsilon}\right\}_{n \geq 0}$ is the following:

(Firstly, we note that $\mathcal{F}^{\varepsilon}\left(\mathcal{G}_{-1}^{\varepsilon}\right)=0$ since $\mathcal{G}_{-1}^{\varepsilon}=0$; secondly, we compute $\rho_{0}^{\varepsilon}$ with (138); thirdly, we compute $\mathcal{G}_{0}^{\varepsilon}$ with (137).

$\rightarrow\left\{\begin{array}{l}\text { Firstly, we compute } \mathcal{F}^{\varepsilon}\left(\mathcal{G}_{0}^{\varepsilon}\right) \text { with (139); } \\ \text { secondly, we compute } \rho_{1}^{\varepsilon} \text { with }(138) ; \\ \text { thirdly, we compute } \mathcal{G}_{1}^{\varepsilon} \text { with }(140)\end{array} \rightarrow \ldots\right.$

$$
\ldots \rightarrow\left\{\begin{array}{l}
\text { Firstly, we compute } \mathcal{F}^{\varepsilon}\left(\mathcal{G}_{n-1}^{\varepsilon}\right) \text { with }(139) ; \\
\text { secondly, we compute } \rho_{n}^{\varepsilon} \text { with }(138) ; \\
\text { thirdly, we compute } \mathcal{G}_{n}^{\varepsilon} \text { with (140). }
\end{array} \rightarrow \ldots\right.
$$

By using (138), we obtain that

$$
\left\{\begin{array}{l}
\partial_{t} \rho_{0}^{\varepsilon}+\partial_{x}\left(u \rho_{0}^{\varepsilon}\right)=0 \quad(\text { constraint (138) with } n=0) \\
\partial_{t} \rho_{1}^{\varepsilon}+\partial_{x}\left(u \rho_{1}^{\varepsilon}\right)=\mathcal{F}^{\varepsilon}\left(\mathcal{G}_{0}^{\varepsilon}\right) \quad(\text { constraint (138) with } n=1) \\
\partial_{t} \rho_{2}^{\varepsilon}+\partial_{x}\left(u \rho_{2}^{\varepsilon}\right)=\mathcal{F}^{\varepsilon}\left(\mathcal{G}_{1}^{\varepsilon}\right) \quad(\text { constraint }(138) \text { with } n=2)
\end{array}\right.
$$

that is to say

$$
\partial_{t} \rho^{\varepsilon}+\partial_{x}\left(u \rho^{\varepsilon}\right)=\sqrt{\varepsilon} \mathcal{F}^{\varepsilon}\left(\mathcal{G}_{0}^{\varepsilon}\right)+\varepsilon \mathcal{F}^{\varepsilon}\left(\mathcal{G}_{1}^{\varepsilon}\right)+O(\varepsilon)
$$

since $\rho^{\varepsilon}=\rho_{0}+\sqrt{\varepsilon} \rho_{1}+\varepsilon \rho_{2}+O\left(\varepsilon^{3 / 2}\right)$. Let us note that the term of order $\varepsilon$ in (143) is obtained by supposing that $\mathcal{F}^{\varepsilon}\left(\mathcal{G}_{n}^{\varepsilon}\right)=O(1 / \sqrt{\varepsilon})(\forall n \geq 0)$ because of the velocity $v_{q}^{\varepsilon}$ in (139). Thus, we obtain (113) by using the following lemma:

Lemma A.2. We have

$$
\left\{\begin{array}{l}
\mathcal{F}^{\varepsilon}\left(\mathcal{G}_{0}^{\varepsilon}\right)=\frac{v}{\sqrt{\varepsilon}} \partial_{x x}^{2} \rho_{0}^{\varepsilon}+O(\sqrt{\varepsilon}), \quad(a) \\
\mathcal{F}^{\varepsilon}\left(\mathcal{G}_{1}^{\varepsilon}\right)=\frac{v}{\sqrt{\varepsilon}} \partial_{x x}^{2} \rho_{1}^{\varepsilon}+O(1)
\end{array}\right.
$$

Moreover, we have the following result which will allow us to obtain (115):

Lemma A.3. We have

$$
\left\{\begin{array}{l}
g_{1, q}^{\varepsilon}=\sqrt{\varepsilon} \partial_{x}\left(u \rho_{0}^{\varepsilon}\right)-(-1)^{q} \sqrt{v} \frac{\partial_{x}\left(m_{q}^{\varepsilon} \rho_{0}^{\varepsilon}\right)}{m_{q}^{\varepsilon}}+\rho_{1}^{\varepsilon}, \\
g_{2, q}^{\varepsilon}=-v\left[\partial_{x x}^{2} \rho_{0}^{\varepsilon}-\frac{\partial_{x x}^{2}\left(m_{q}^{\varepsilon} \rho_{0}^{\varepsilon}\right)}{m_{q}^{\varepsilon}}\right]-(-1)^{q} \sqrt{v} \frac{\partial_{x}\left(m_{q}^{\varepsilon} \rho_{1}^{\varepsilon}\right)}{m_{q}^{\varepsilon}}+\rho_{2}^{\varepsilon}+O(\sqrt{\varepsilon}) .
\end{array}\right.
$$

Thus, by using (145), we obtain

$$
\begin{aligned}
m_{q}^{\varepsilon}\left(g_{0, q}^{\varepsilon}+\sqrt{\varepsilon} g_{1, q}^{\varepsilon}+\varepsilon g_{2, q}^{\varepsilon}\right)= & \frac{1}{2}\left(1+(-1)^{q} \sqrt{\frac{\varepsilon}{v}} u\right) \times \\
& \times\left\{\rho_{0}^{\varepsilon}+\sqrt{\varepsilon} \rho_{1}^{\varepsilon}+\varepsilon \rho_{2}^{\varepsilon}-(-1)^{q} \frac{\sqrt{\varepsilon v}}{m_{q}^{\varepsilon}} \partial_{x}\left[m_{q}^{\varepsilon}\left(\rho_{0}^{\varepsilon}+\sqrt{\varepsilon} \rho_{1}^{\varepsilon}\right)\right]\right. \\
& \left.+\varepsilon \partial_{x}\left(u \rho_{0}^{\varepsilon}\right)-\varepsilon v\left[\partial_{x x}^{2} \rho_{0}-\frac{\partial_{x x}^{2}\left(m_{q}^{\varepsilon} \rho_{0}^{\varepsilon}\right)}{m_{q}^{\varepsilon}}\right]\right\}+O\left(\varepsilon^{3 / 2}\right) .
\end{aligned}
$$


By noting that $\partial_{x} m_{q}^{\varepsilon}=(-1)^{q} \sqrt{\frac{\varepsilon}{v}} \cdot \frac{u^{\prime}(x)}{2}$, we deduce from the previous equality that

$$
\begin{aligned}
m_{q}^{\varepsilon}\left(g_{0, q}^{\varepsilon}+\sqrt{\varepsilon} g_{1, q}^{\varepsilon}+\varepsilon g_{2, q}^{\varepsilon}\right)= & \frac{1}{2}\left(1+(-1)^{q} \sqrt{\frac{\varepsilon}{v}} u\right) \times \\
& \times\left[\rho_{0}^{\varepsilon}+\sqrt{\varepsilon} \rho_{1}^{\varepsilon}+\varepsilon \rho_{2}^{\varepsilon}-(-1)^{q} \sqrt{\varepsilon v} \partial_{x}\left(\rho_{0}^{\varepsilon}+\sqrt{\varepsilon} \rho_{1}^{\varepsilon}\right)-\varepsilon \rho_{0}^{\varepsilon} \frac{u^{\prime}(x)}{2 m_{q}^{\varepsilon}}+\varepsilon \partial_{x}\left(u \rho_{0}^{\varepsilon}\right)\right]+O\left(\varepsilon^{3 / 2}\right) \\
= & \frac{1}{2}\left(\rho_{0}^{\varepsilon}+\sqrt{\varepsilon} \rho_{1}^{\varepsilon}+\varepsilon \rho_{2}^{\varepsilon}\right)\left(1+(-1)^{q} \sqrt{\frac{\varepsilon}{v} u}\right) \\
& -(-1)^{q} \frac{\sqrt{\varepsilon v}}{2} \partial_{x}\left(\rho_{0}^{\varepsilon}+\sqrt{\varepsilon} \rho_{1}^{\varepsilon}\right)-\frac{\varepsilon}{2}\left[\rho_{0}^{\varepsilon} \frac{u^{\prime}(x)}{2 m_{q}^{\varepsilon}}+u \partial_{x} \rho_{0}^{\varepsilon}+\partial_{x}\left(u \rho_{0}^{\varepsilon}\right)\right]+O\left(\varepsilon^{3 / 2}\right)
\end{aligned}
$$

that is to say

$$
f_{q}^{\varepsilon}=\frac{\rho^{\varepsilon}}{2}\left(1+(-1)^{q} \sqrt{\frac{\varepsilon}{v}} u\right)-(-1)^{q} \frac{\sqrt{\varepsilon v}}{2} \partial_{x} \rho^{\varepsilon}-\frac{\varepsilon}{2}\left[\rho_{0}^{\varepsilon} \frac{u^{\prime}(x)}{2 m_{q}^{\varepsilon}}+u \partial_{x} \rho_{0}^{\varepsilon}-\partial_{x}\left(u \rho_{0}^{\varepsilon}\right)\right]+O\left(\varepsilon^{3 / 2}\right) .
$$

Since $\rho_{0}^{\varepsilon} \frac{u^{\prime}(x)}{2 m_{q}^{\varepsilon}}+u \partial_{x} \rho_{0}^{\varepsilon}=\partial_{x}\left(u \rho_{0}^{\varepsilon}\right)+O(\sqrt{\varepsilon})$, we obtain

$$
f_{q}^{\varepsilon}(t, x)=\frac{\rho^{\varepsilon}}{2}\left[1+(-1)^{q} \sqrt{\varepsilon}\left(\frac{u(x)}{\sqrt{v}}-\sqrt{v} \frac{\partial_{x} \rho^{\varepsilon}}{\rho^{\varepsilon}}\right)\right]+O\left(\varepsilon^{3 / 2}\right)
$$

which is exactly the expansion (115).

It remains to prove Lemmae A.1, A.2 and A.3:

Proof of lemma A.1: The matrix $\mathbf{A}^{\varepsilon}$ is not invertible and its kernel is given by

$$
\operatorname{Ker} \mathbf{A}^{\varepsilon}=\left\{X \in \mathbb{R}^{2} \quad \text { such that } \quad X=\mu(1,1)^{T}, \quad \mu \in \mathbb{R}\right\} .
$$

Moreover, $\mathbf{A}^{\varepsilon}$ admits the eigenvalue $\lambda=-1$ whose eigenspace is given by

$$
\begin{aligned}
\mathcal{E}^{\varepsilon, \lambda=-1} & =\left\{X \in \mathbb{R}^{2} \quad \text { such that } \quad X=\mu\left(m_{2}^{\varepsilon},-m_{1}^{\varepsilon}\right)^{T}, \quad \mu \in \mathbb{R}\right\} \\
& =\left\{X \in \mathbb{R}^{2} \quad \text { such that } \quad \sum_{q \in\{1,2\}} X_{q} m_{q}^{\varepsilon}=0\right\}
\end{aligned}
$$

Let us note that $\mathcal{E}^{\varepsilon, \lambda=-1}$ depends on $\varepsilon$ - which is not the case of $\operatorname{Ker} \mathbf{A}^{\varepsilon}$-, that $\operatorname{Ker} \mathbf{A}^{\varepsilon} \oplus \mathcal{E}^{\varepsilon, \lambda=-1}=\mathbb{R}^{2}$ and that $\mathcal{E}^{\varepsilon, \lambda=-1} \perp\left(m_{1}^{\varepsilon}, m_{2}^{\varepsilon}\right)^{T}$. The linear application $\mathbf{A}^{\varepsilon}: X \mapsto \mathbf{A}^{\varepsilon} \cdot X$ defines a bijection from $\mathcal{E}^{\varepsilon, \lambda=-1}$ into $\mathcal{E}^{\varepsilon, \lambda=-1}$. Thus, we can solve linear system (135) if and only if

$$
\forall n \geq 0: \quad \mathcal{B}^{\varepsilon}\left(\mathcal{G}_{n}^{\varepsilon}\right) \in \mathcal{E}^{\varepsilon, \lambda=-1} .
$$

This corresponds to the Fredholm alternative in finite dimension. Thus, by using (147)(b), the vector $\mathcal{B}^{\varepsilon}\left(\mathcal{G}_{n}^{\varepsilon}\right)$ has to verify the constraint

$$
\forall n \geq 0: \quad \sum_{q \in\{1,2\}} m_{q}^{\varepsilon} b_{q}^{\varepsilon}\left(\mathcal{G}_{n}^{\varepsilon}\right)=0
$$


that is to say

$$
\forall n \geq 0: \quad \sum_{q \in\{1,2\}}\left[\partial_{t}\left(m_{q}^{\varepsilon} g_{n, q}^{\varepsilon}\right)+v_{q}^{\varepsilon} \partial_{x}\left(m_{q}^{\varepsilon} g_{n, q}^{\varepsilon}\right)\right]=0
$$

which is equivalent to

$$
\forall n \geq 0: \quad \partial_{t} \rho_{n}^{\varepsilon}+\partial_{x}\left(u \rho_{n}^{\varepsilon}\right)=-\partial_{x}\left[\sum_{q \in\{1,2\}}\left(v_{q}^{\varepsilon}-u\right) m_{q}^{\varepsilon} g_{n, q}^{\varepsilon}\right]
$$

by using (132). Moreover, we have

$$
\forall n \geq 0: \quad \mathbf{A}^{\varepsilon} \cdot \mathcal{G}_{n+1}^{\varepsilon}=\mathcal{B}^{\varepsilon}\left(\mathcal{G}_{n}^{\varepsilon}\right) \quad \text { and } \quad \mathcal{B}^{\varepsilon}\left(\mathcal{G}_{n}^{\varepsilon}\right) \in \mathcal{E}^{\varepsilon, \lambda=-1} \quad \Longrightarrow \quad \mathcal{G}_{n+1}^{\varepsilon}=-\mathcal{B}^{\varepsilon}\left(\mathcal{G}_{n}^{\varepsilon}\right)+\mu_{n+1}\left(\begin{array}{l}
1 \\
1
\end{array}\right)
$$

where $\mu_{n+1} \in \mathbb{R}$. Thus, by using (132) and (149), we obtain $\rho_{n+1}^{\varepsilon}=\sum_{q \in\{1,2\}} m_{q}^{\varepsilon} g_{n+1, q}^{\varepsilon}=0+\mu_{n+1}$ which implies that $\mathcal{G}_{n+1}^{\varepsilon}$ is given by (140). As a consequence, we have

$$
\begin{aligned}
-\partial_{x}\left[\sum_{q \in\{1,2\}}\left(v_{q}^{\varepsilon}-u\right) m_{q}^{\varepsilon} g_{n, q}^{\varepsilon}\right] & =-\partial_{x}\left\{\sum_{q \in\{1,2\}}\left(v_{q}^{\varepsilon}-u\right) m_{q}^{\varepsilon}\left[-b_{q}^{\varepsilon}\left(\mathcal{G}_{n-1}^{\varepsilon}\right)+\rho_{n}^{\varepsilon}\right]\right\} \\
& =\mathcal{F}^{\varepsilon}\left(\mathcal{G}_{n-1}^{\varepsilon}\right)+0
\end{aligned}
$$

by using the fact that

$$
\sum_{q \in\{1,2\}}\left(\begin{array}{c}
1 \\
v_{q}^{\varepsilon}
\end{array}\right) m_{q}^{\varepsilon}=\left(\begin{array}{c}
1 \\
u
\end{array}\right)
$$

and Definition (139), which allows to obtain (138) by taking into account (150). Finally, we have proven that (135) admits a solution $\left\{\mathcal{G}_{n}^{\varepsilon}\right\}_{n \geq 0}$ under the constraints (138). Moreover, this solution is unique since (138) are linear PDEs which admit an unique solution.

Proof of Lemmae A.2 and A.3: We firstly prove (144)(a) and (145)(a); then, we prove (144)(b) and (145)(b). We have

$$
\begin{aligned}
\mathcal{F}^{\varepsilon}\left(\mathcal{G}_{0}\right) & =\partial_{x}\left[\sum_{q \in\{1,2\}}\left(v_{q}^{\varepsilon}-u\right) m_{q}^{\varepsilon} b_{q}^{\varepsilon}\left(\mathcal{G}_{0}^{\varepsilon}\right)\right]=\sqrt{\varepsilon} \partial_{x}\left[\sum_{q \in\{1,2\}}\left(v_{q}^{\varepsilon}-u\right) m_{q}^{\varepsilon} \partial_{t} \rho_{0}^{\varepsilon}\right]+\sqrt{\varepsilon} \partial_{x}\left[\sum_{q \in\{1,2\}}\left(v_{q}^{\varepsilon}-u\right) v_{q}^{\varepsilon} \partial_{x}\left(m_{q}^{\varepsilon} \rho_{0}^{\varepsilon}\right)\right] \\
& =0+\sqrt{\varepsilon} \partial_{x}\left[\sum_{q \in\{1,2\}} v_{q}^{\varepsilon} \partial_{x}\left(m_{q}^{\varepsilon} \rho_{0}^{\varepsilon}\right)\right]-\sqrt{\varepsilon} \partial_{x}\left[u \partial_{x}\left(\sum_{q \in\{1,2\}} v_{q}^{\varepsilon} m_{q}^{\varepsilon} \rho_{0}^{\varepsilon}\right)\right] \\
& =\frac{v}{\sqrt{\varepsilon}} \partial_{x}\left[\sum_{q \in\{1,2\}} \partial_{x}\left(m_{q}^{\varepsilon} \rho_{0}^{\varepsilon}\right)\right]-\sqrt{\varepsilon} \partial_{x}\left[u \partial_{x}\left(u \rho_{0}^{\varepsilon}\right)\right]=\frac{v}{\sqrt{\varepsilon}} \partial_{x x}^{2} \rho_{0}^{\varepsilon}+O(\sqrt{\varepsilon})
\end{aligned}
$$

which gives (144)(a). Moreover, we have

$$
\begin{aligned}
b_{q}^{\varepsilon}\left(\mathcal{G}_{0}^{\varepsilon}\right) & =\sqrt{\varepsilon} \frac{\partial_{t}\left(m_{q}^{\varepsilon} \rho_{0}^{\varepsilon}\right)}{m_{q}^{\varepsilon}}+\sqrt{\varepsilon} v_{q}^{\varepsilon} \frac{\partial_{x}\left(m_{q}^{\varepsilon} \rho_{0}^{\varepsilon}\right)}{m_{q}^{\varepsilon}}=\sqrt{\varepsilon} \partial_{t} \rho_{0}^{\varepsilon}+(-1)^{q} \sqrt{v} \frac{\partial_{x}\left(m_{q}^{\varepsilon} \rho_{0}^{\varepsilon}\right)}{m_{q}^{\varepsilon}} \\
& =-\sqrt{\varepsilon} \partial_{x}\left(u \rho_{0}^{\varepsilon}\right)+(-1)^{q} \sqrt{v} \frac{\partial_{x}\left(m_{q}^{\varepsilon} \rho_{0}^{\varepsilon}\right)}{m_{q}^{\varepsilon}}
\end{aligned}
$$


by using (142)(a). We obtain (145)(a) by using (140). In the same way, we have

$$
\begin{aligned}
\mathcal{F}^{\varepsilon}\left(\mathcal{G}_{1}^{\varepsilon}\right) & =\partial_{x}\left[\sum_{q \in\{1,2\}}\left(v_{q}^{\varepsilon}-u\right) m_{q}^{\varepsilon} b_{q}^{\varepsilon}\left(\mathcal{G}_{1}\right)\right]=\sqrt{\varepsilon} \partial_{x}\left[\sum_{q \in\{1,2\}}\left(v_{q}^{\varepsilon}-u\right) m_{q}^{\varepsilon} \partial_{t} g_{1, q}^{\varepsilon}\right]+\sqrt{\varepsilon} \partial_{x}\left[\sum_{q \in\{1,2\}}\left(v_{q}^{\varepsilon}-u\right) v_{q}^{\varepsilon} \partial_{x}\left(m_{q}^{\varepsilon} g_{1, q}^{\varepsilon}\right)\right] \\
& =\sqrt{\varepsilon} \partial_{x}\left[\sum_{q \in\{1,2\}}\left(v_{q}^{\varepsilon}-u\right) m_{q}^{\varepsilon} \partial_{t} g_{1, q}^{\varepsilon}\right]+\sqrt{\varepsilon} \partial_{x}\left[\sum_{q \in\{1,2\}} v_{q}^{\varepsilon 2} \partial_{x}\left(m_{q}^{\varepsilon} g_{1, q}^{\varepsilon}\right)\right]-\sqrt{\varepsilon} \partial_{x}\left[u \partial_{x}\left[\sum_{q \in\{1,2\}} v_{q}^{\varepsilon} m_{q}^{\varepsilon} g_{1, q}^{\varepsilon}\right)\right] \\
& =\sqrt{\varepsilon} \partial_{x}\left[\sum_{q \in\{1,2\}}\left(v_{q}^{\varepsilon}-u\right) m_{q}^{\varepsilon} \partial_{t} g_{1, q}^{\varepsilon}\right]+\frac{v}{\sqrt{\varepsilon}} \partial_{x}\left[\sum_{q \in\{1,2\}} \partial_{x}\left(m_{q}^{\varepsilon} g_{1, q}^{\varepsilon}\right)\right]+O(1) \\
& =\sqrt{\varepsilon} \partial_{x}\left[\sum_{q \in\{1,2\}}\left(v_{q}^{\varepsilon}-u\right) m_{q}^{\varepsilon} \partial_{t} g_{1, q}^{\varepsilon}\right]+\frac{v}{\sqrt{\varepsilon}} \partial_{x x}^{2} \rho_{1}^{\varepsilon}+O(1) .
\end{aligned}
$$

But, by using (145)(a), we have also

$$
\begin{aligned}
\sqrt{\varepsilon} \partial_{x}\left[\sum_{q \in\{1,2\}}\left(v_{q}^{\varepsilon}-u\right) m_{q}^{\varepsilon} \partial_{t} g_{1, q}^{\varepsilon}\right] & =\sqrt{\varepsilon} \partial_{t x}^{2} \sum_{q \in\{1,2\}}\left(v_{q}^{\varepsilon}-u\right) m_{q}^{\varepsilon}\left[\sqrt{\varepsilon} \partial_{x}\left(u \rho_{0}^{\varepsilon}\right)-(-1)^{q} \sqrt{v} \frac{\partial_{x}\left(m_{q}^{\varepsilon} \rho_{0}^{\varepsilon}\right)}{m_{q}^{\varepsilon}}+\rho_{1}^{\varepsilon}\right] \\
& =0-\sqrt{\varepsilon v} \partial_{t x}^{2} \sum_{q \in\{1,2\}}(-1)^{q}\left(v_{q}^{\varepsilon}-u\right) \partial_{x}\left(m_{q}^{\varepsilon} \rho_{0}^{\varepsilon}\right)=O(1) .
\end{aligned}
$$

Thus, we can write that

$$
\mathcal{F}^{\varepsilon}\left(\mathcal{G}_{1}^{\varepsilon}\right)=\frac{v}{\sqrt{\varepsilon}} \partial_{x x}^{2} \rho_{1}^{\varepsilon}+O(1)
$$

which gives (144)(b). Moreover, by taking into account (145)(a), we obtain

$$
\begin{aligned}
b_{q}^{\varepsilon}\left(\mathcal{G}_{1}^{\varepsilon}\right)= & \sqrt{\varepsilon} \frac{\partial_{t}\left(m_{q}^{\varepsilon} g_{1, q}^{\varepsilon}\right)}{m_{q}^{\varepsilon}}+\sqrt{\varepsilon} v_{q}^{\varepsilon} \frac{\partial_{x}\left(m_{q}^{\varepsilon} g_{1, q}^{\varepsilon}\right)}{m_{q}^{\varepsilon}} \\
= & \sqrt{\varepsilon} \partial_{t}\left(\sqrt{\varepsilon} \partial_{x}\left(u \rho_{0}^{\varepsilon}\right)-(-1)^{q} \sqrt{v} \frac{\partial_{x}\left(m_{q}^{\varepsilon} \rho_{0}^{\varepsilon}\right)}{m_{q}^{\varepsilon}}+\rho_{1}^{\varepsilon}\right) \\
& +(-1)^{q} \sqrt{v} \frac{\partial_{x}\left[m_{q}^{\varepsilon}\left(\sqrt{\varepsilon} \partial_{x}\left(u \rho_{0}^{\varepsilon}\right)-(-1)^{q} \sqrt{v} \frac{\partial_{x}\left(m_{q}^{\varepsilon} \rho_{0}^{\varepsilon}\right)}{m_{q}^{\varepsilon}}+\rho_{1}^{\varepsilon}\right)\right]}{m_{q}^{\varepsilon}} \\
= & \sqrt{\varepsilon} \partial_{t} \rho_{1}^{\varepsilon}-v \frac{\partial_{x x}^{2}\left(m_{q}^{\varepsilon} \rho_{0}^{\varepsilon}\right)}{m_{q}^{\varepsilon}}+(-1)^{q} \sqrt{v} \frac{\partial_{x}\left(m_{q}^{\varepsilon} \rho_{1}^{\varepsilon}\right)}{m_{q}^{\varepsilon}}+O(\sqrt{\varepsilon}) \\
= & v \partial_{x x}^{2} \rho_{0}^{\varepsilon}-v \frac{\partial_{x x}^{2}\left(m_{q}^{\varepsilon} \rho_{0}^{\varepsilon}\right)}{m_{q}^{\varepsilon}}+(-1)^{q} \sqrt{v} \frac{\partial_{x}\left(m_{q}^{\varepsilon} \rho_{1}^{\varepsilon}\right)}{m_{q}^{\varepsilon}}+O(\sqrt{\varepsilon})
\end{aligned}
$$

by also using (142)(b) and (144)(a). Then, we obtain (145)(b) by using (140). 


\section{B. The LBM scheme written in function of $f_{q}$ when $u(x)=0$}

When $u(x)=0$, the LBM scheme (35) is given by

$$
\left\{\begin{array}{l}
g_{1, i}^{n+1}=g_{1, i+1}^{n}\left(1-\frac{\eta}{2}\right)+g_{2, i+1}^{n} \frac{\eta}{2}, \\
g_{2, i}^{n+1}=g_{2, i-1}^{n}\left(1-\frac{\eta}{2}\right)+g_{1, i-1}^{n} \frac{\eta}{2} \\
\rho_{i}^{n+1}=g_{1, i}^{n+1}+g_{2, i}^{n+1} .
\end{array}\right.
$$

On the other side, by using (20), we have $g_{q}=f_{q}-\frac{\Delta t}{2 \varepsilon}\left(M_{q}-f_{q}\right)$ that is to say

$$
\left\{\begin{array}{l}
g_{1}=f_{1}\left(1+\frac{1}{4 C_{d}}\right)-\frac{f_{2}}{4 C_{d}} \\
g_{2}=f_{2}\left(1+\frac{1}{4 C_{d}}\right)-\frac{f_{1}}{4 C_{d}}
\end{array}\right.
$$

since $\varepsilon=C_{d} \Delta t$ and $M_{1}=\frac{f_{1}+f_{2}}{2}$. By injecting (152) in (151), we obtain

$$
A\left(\begin{array}{l}
f_{1} \\
f_{2}
\end{array}\right)_{i}^{n+1}=b
$$

with

$$
A=\left(\begin{array}{cc}
1+\frac{1}{4 C_{d}} & -\frac{1}{4 C_{d}} \\
-\frac{1}{4 C_{d}} & 1+\frac{1}{4 C_{d}}
\end{array}\right)
$$

and

$$
b=\left(\begin{array}{l}
{\left[f_{1, i+1}^{n}\left(1+\frac{1}{4 C_{d}}\right)-\frac{f_{2, i+1}^{n}}{4 C_{d}}\right] \cdot\left(1-\frac{\eta}{2}\right)+\left[f_{2, i+1}^{n}\left(1+\frac{1}{4 C_{d}}\right)-\frac{f_{1, i+1}^{n}}{4 C_{d}}\right] \cdot \frac{\eta}{2}} \\
{\left[f_{2, i-1}^{n}\left(1+\frac{1}{4 C_{d}}\right)-\frac{f_{1, i-1}^{n}}{4 C_{d}}\right] \cdot\left(1-\frac{\eta}{2}\right)+\left[f_{1, i-1}^{n}\left(1+\frac{1}{4 C_{d}}\right)-\frac{f_{2, i-1}^{n}}{4 C_{d}}\right] \cdot \frac{\eta}{2}}
\end{array}\right) .
$$

By using the fact that

$$
A^{-1}=\frac{1}{C_{d}+1 / 2}\left(\begin{array}{cc}
C_{d}+1 / 4 & 1 / 4 \\
1 / 4 & C_{d}+1 / 4
\end{array}\right)
$$

and that $\eta=\frac{1}{C_{d}+1 / 2}$, we obtain

$$
\begin{aligned}
f_{1, i}^{n+1}= & \frac{C_{d}+1 / 4}{\left(C_{d}+1 / 2\right)^{2}}\left\{C_{d}\left[f_{1, i+1}^{n}\left(1+\frac{1}{4 C_{d}}\right)-\frac{f_{2, i+1}^{n}}{4 C_{d}}\right]+\frac{1}{2}\left[f_{2, i+1}^{n}\left(1+\frac{1}{4 C_{d}}\right)-\frac{f_{1, i+1}^{n}}{4 C_{d}}\right]\right\} \\
& +\frac{1}{4\left(C_{d}+1 / 2\right)^{2}}\left\{C_{d}\left[f_{2, i-1}^{n}\left(1+\frac{1}{4 C_{d}}\right)-\frac{f_{1, i-1}^{n}}{4 C_{d}}\right]+\frac{1}{2}\left[f_{1, i-1}^{n}\left(1+\frac{1}{4 C_{d}}\right)-\frac{f_{2, i-1}^{n}}{4 C_{d}}\right]\right\}
\end{aligned}
$$


that is to say

$$
\begin{aligned}
f_{1, i}^{n+1}= & \frac{4 C_{d}+1}{4\left(C_{d}+1 / 2\right)^{2}}\left\{f_{1, i+1}^{n} \cdot \frac{8 C_{d}^{2}+2 C_{d}-1}{8 C_{d}}+f_{2, i+1}^{n} \cdot \frac{C_{d}+1 / 2}{4 C_{d}}\right\} \\
& +\frac{1}{4\left(C_{d}+1 / 2\right)^{2}}\left\{f_{2, i-1}^{n} \cdot \frac{8 C_{d}^{2}+2 C_{d}-1}{8 C_{d}}+f_{1, i-1}^{n} \cdot \frac{C_{d}+1 / 2}{4 C_{d}}\right\} .
\end{aligned}
$$

By noting that $8 C_{d}^{2}+2 C_{d}-1=2\left(4 C_{d}-1\right) \cdot\left(C_{d}+1 / 2\right)$, we finally obtain

$$
f_{1, i}^{n+1}=\frac{f_{1, i+1}^{n}\left(16 C_{d}^{2}-1\right)+f_{2, i+1}^{n}\left(4 C_{d}+1\right)+f_{2, i-1}^{n}\left(4 C_{d}-1\right)+f_{1, i-1}^{n}}{16 C_{d}\left(C_{d}+\frac{1}{2}\right)}
$$

which gives (36)(a). We obtain (36)(b) by symmetry.

\section{Proof of Property 3.1}

The scheme (37) is equivalent to the scheme

$$
\left\{\begin{array}{l}
g_{1, i+1}^{n}=g_{1, i}^{n+1}(1-\hat{\eta})+M_{1, i}^{n+1} \hat{\eta} \\
g_{2, i-1}^{n}=g_{2, i}^{n+1}(1-\hat{\eta})+M_{2, i}^{n+1} \hat{\eta}
\end{array}\right.
$$

that is to say to the scheme

$$
\left\{\begin{array}{l}
g_{1, i+1}^{n}=g_{1, i}^{n+1}\left[1-\frac{\hat{\eta}}{2}\left(1+\frac{\Delta t}{\Delta x} u\left(x_{i}\right)\right)\right]+g_{2, i}^{n+1} \frac{\hat{\eta}}{2}\left(1-\frac{\Delta t}{\Delta x} u\left(x_{i}\right)\right) \\
g_{2, i-1}^{n}=g_{2, i}^{n+1}\left[1-\frac{\hat{\eta}}{2}\left(1-\frac{\Delta t}{\Delta x} u\left(x_{i}\right)\right)\right]+g_{1, i}^{n+1} \frac{\hat{\eta}}{2}\left(1+\frac{\Delta t}{\Delta x} u\left(x_{i}\right)\right)
\end{array}\right.
$$

since $M_{q, i}=\frac{g_{1, i}+g_{2, i}}{2}\left(1+(-1)^{q} \frac{\Delta t}{\Delta x} u\left(x_{i}\right)\right)$. We end the proof by noting that

$$
\left(\begin{array}{cc}
1-\frac{\hat{\eta}}{2}\left(1+\frac{\Delta t}{\Delta x} u\left(x_{i}\right)\right) & \frac{\hat{\eta}}{2}\left(1-\frac{\Delta t}{\Delta x} u\left(x_{i}\right)\right) \\
\frac{\hat{\eta}}{2}\left(1+\frac{\Delta t}{\Delta x} u\left(x_{i}\right)\right) & 1-\frac{\hat{\eta}}{2}\left(1-\frac{\Delta t}{\Delta x} u\left(x_{i}\right)\right)
\end{array}\right)^{-1}=\left(\begin{array}{rr}
1-\frac{\eta}{2}\left(1+\frac{\Delta t}{\Delta x} u\left(x_{i}\right)\right) & \frac{\eta}{2}\left(1-\frac{\Delta t}{\Delta x} u\left(x_{i}\right)\right) \\
\frac{\eta}{2}\left(1+\frac{\Delta t}{\Delta x} u\left(x_{i}\right)\right) & 1-\frac{\eta}{2}\left(1-\frac{\Delta t}{\Delta x} u\left(x_{i}\right)\right)
\end{array}\right)
$$

which comes from the fact that $\hat{\eta}+\eta=\hat{\eta} \eta$.

\section{Proof of Lemmae 4.1, 4.2 and 4.3}

In the following proof, we firstly focus on the $\mathrm{LBM}^{*}$ scheme. Then, we focus on the LBM scheme which is less easy to study. 


\section{Proof of Lemma 4.1:}

- Study of the LBM* scheme:

We deduce from the $\mathrm{LBM}^{*}$ scheme (38) that $\rho_{i}^{n+1}=g_{1, i+1}^{n}+g_{2, i-1}^{n}(n \geq 0)$. Thus, by applying again (38), we find

$$
\begin{aligned}
\rho_{i}^{n+1}= & g_{1, i+2}^{n-1}\left[1-\frac{\eta}{2}\left(1+\frac{\Delta t}{\Delta x} u\left(x_{i+1}\right)\right)\right]+g_{2, i}^{n-1} \frac{\eta}{2}\left(1-\frac{\Delta t}{\Delta x} u\left(x_{i+1}\right)\right) \\
& +g_{2, i-2}^{n-1}\left[1-\frac{\eta}{2}\left(1-\frac{\Delta t}{\Delta x} u\left(x_{i-1}\right)\right)\right]+g_{1, i}^{n-1} \frac{\eta}{2}\left(1+\frac{\Delta t}{\Delta x} u\left(x_{i-1}\right)\right) \quad \text { with } \quad n \geq 1 .
\end{aligned}
$$

By noting that

$$
\left\{\begin{array}{l}
\rho_{i+1}^{n}=g_{1, i+2}^{n-1}+g_{2, i}^{n-1}, \\
\rho_{i-1}^{n}=g_{1, i}^{n-1}+g_{2, i-2}^{n-1}
\end{array} \quad \text { with } \quad n \geq 1,\right.
$$

we deduce from (153) that

$$
\begin{aligned}
\rho_{i}^{n+1}= & \left(\rho_{i+1}^{n}-g_{2, i}^{n-1}\right)\left(1-\frac{\eta}{2}\right)+g_{2, i}^{n-1} \frac{\eta}{2}+\left(\rho_{i-1}^{n}-g_{1, i}^{n-1}\right)\left(1-\frac{\eta}{2}\right)+g_{1, i}^{n-1} \frac{\eta}{2} \\
& -\frac{\eta}{2} \cdot \frac{\Delta t}{\Delta x}\left[u\left(x_{i+1}\right) \rho_{i+1}^{n}-u\left(x_{i-1}\right) \rho_{i-1}^{n}\right] \quad \text { with } \quad n \geq 1
\end{aligned}
$$

that is to say

$$
\rho_{i}^{n+1}=\rho_{i+1}^{n}\left(1-\frac{\eta}{2}\right)+\rho_{i-1}^{n}\left(1-\frac{\eta}{2}\right)+\rho_{i}^{n-1}(\eta-1)-\frac{\eta}{2} \cdot \frac{\Delta t}{\Delta x}\left[u\left(x_{i+1}\right) \rho_{i+1}^{n}-u\left(x_{i-1}\right) \rho_{i-1}^{n}\right] \quad \text { with } \quad n \geq 1 .
$$

By using the fact that $\eta=\frac{1}{C_{d}+\frac{1}{2}}$, we obtain

$$
\left(2 C_{d}+1\right) \rho_{i}^{n+1}=2 C_{d}\left(\rho_{i+1}^{n}+\rho_{i-1}^{n}\right)+\left(1-2 C_{d}\right) \rho_{i}^{n-1}-\frac{\Delta t}{\Delta x}\left[u\left(x_{i+1}\right) \rho_{i+1}^{n}-u\left(x_{i-1}\right) \rho_{i-1}^{n}\right] \quad \text { with } \quad n \geq 1
$$

that is to say

$$
\rho_{i}^{n+1}-\rho_{i}^{n-1}=2 C_{d}\left(\rho_{i+1}^{n}-\rho_{i}^{n+1}-\rho_{i}^{n-1}+\rho_{i-1}^{n}\right)-\frac{\Delta t}{\Delta x}\left[u\left(x_{i+1}\right) \rho_{i+1}^{n}-u\left(x_{i-1}\right) \rho_{i-1}^{n}\right] \quad \text { with } \quad n \geq 1
$$

which is equivalent to

$$
\frac{\rho_{i}^{n+1}-\rho_{i}^{n-1}}{2 \Delta t}=\frac{v}{\Delta x^{2}}\left(\rho_{i+1}^{n}-\rho_{i}^{n+1}-\rho_{i}^{n-1}+\rho_{i-1}^{n}\right)-\frac{1}{2 \Delta x}\left[u\left(x_{i+1}\right) \rho_{i+1}^{n}-u\left(x_{i-1}\right) \rho_{i-1}^{n}\right] \quad \text { with } \quad n \geq 1 .
$$

We conclude the proof by noting that

$$
\left\{\begin{array}{l}
g_{1, i}^{0}=\rho_{i}^{0} \cdot\left[(1-\alpha)-\beta \frac{\Delta t}{\Delta x} u\left(x_{i}\right)\right], \\
g_{2, i}^{0}=\rho_{i}^{0} \cdot\left[\alpha+\beta \frac{\Delta t}{\Delta x} u\left(x_{i}\right)\right]
\end{array}\right.
$$


coupled to the $\mathrm{LBM}^{*}$ scheme (38) implies that

$$
\rho_{i}^{n=1}:=\alpha \rho_{i-1}^{0}+(1-\alpha) \rho_{i+1}^{0}-\beta \frac{\Delta t}{\Delta x}\left[\rho_{i+1}^{0} u\left(x_{i+1}\right)-\rho_{i-1}^{0} u\left(x_{i-1}\right)\right] .
$$

- Study of the LBM scheme:

We deduce from the LBM scheme (35) that

$$
\rho_{i}^{n+1}=\left(g_{1, i+1}^{n}+g_{2, i-1}^{n}\right)\left(1-\frac{\eta}{2}\right)+\left(g_{1, i-1}^{n}+g_{2, i+1}^{n}\right) \frac{\eta}{2}-\frac{\eta}{2} \cdot \frac{\Delta t}{\Delta x}\left[u\left(x_{i+1}\right) \rho_{i+1}^{n}-u\left(x_{i-1}\right) \rho_{i-1}^{n}\right] .
$$

Thus, by applying again (35), we find

$$
\begin{aligned}
\rho_{i}^{n+1}= & {\left[g_{1, i+2}^{n-1}\left(1-\frac{\eta}{2}\right)+g_{2, i+2}^{n-1} \frac{\eta}{2}-\frac{\rho_{i+2}^{n-1}}{2} \cdot \eta \frac{\Delta t}{\Delta x} u\left(x_{i+2}\right)+g_{2, i-2}^{n-1}\left(1-\frac{\eta}{2}\right)+g_{1, i-2}^{n-1} \frac{\eta}{2}+\frac{\rho_{i-2}^{n-1}}{2} \cdot \eta \frac{\Delta t}{\Delta x} u\left(x_{i-2}\right)\right]\left(1-\frac{\eta}{2}\right) } \\
& {\left[g_{1, i}^{n-1}\left(1-\frac{\eta}{2}\right)+g_{2, i}^{n-1} \frac{\eta}{2}-\frac{\rho_{i}^{n-1}}{2} \cdot \eta \frac{\Delta t}{\Delta x} u\left(x_{i}\right)+g_{2, i}^{n-1}\left(1-\frac{\eta}{2}\right)+g_{1, i}^{n-1} \frac{\eta}{2}+\frac{\rho_{i}^{n-1}}{2} \cdot \eta \frac{\Delta t}{\Delta x} u\left(x_{i}\right)\right] \frac{\eta}{2} } \\
& -\frac{\eta}{2} \cdot \frac{\Delta t}{\Delta x}\left[u\left(x_{i+1}\right) \rho_{i+1}^{n}-u\left(x_{i-1}\right) \rho_{i-1}^{n}\right] \quad \text { with } \quad n \geq 1
\end{aligned}
$$

which is equivalent to

$$
\begin{aligned}
\rho_{i}^{n+1}= & {\left[\left(g_{1, i+2}^{n-1}+g_{2, i}^{n-1}\right)\left(1-\frac{\eta}{2}\right)+\left(g_{1, i}^{n-1}+g_{2, i+2}^{n-1}\right) \frac{\eta}{2}-\frac{\eta}{2} \cdot \frac{\Delta t}{\Delta x}\left(u\left(x_{i+2}\right) \rho_{i+2}^{n-1}-u\left(x_{i}\right) \rho_{i}^{n-1}\right)\right]\left(1-\frac{\eta}{2}\right) } \\
& +\left[\left(g_{1, i}^{n-1}+g_{2, i-2}^{n-1}\right)\left(1-\frac{\eta}{2}\right)+\left(g_{1, i-2}^{n-1}+g_{2, i}^{n-1}\right) \frac{\eta}{2}-\frac{\eta}{2} \cdot \frac{\Delta t}{\Delta x}\left(u\left(x_{i}\right) \rho_{i}^{n-1}-u\left(x_{i-2}\right) \rho_{i-2}^{n-1}\right)\right]\left(1-\frac{\eta}{2}\right) \\
& +\left(g_{1, i}^{n-1}+g_{2, i}^{n-1}\right)(\eta-1)-\frac{\eta}{2} \cdot \frac{\Delta t}{\Delta x}\left(u\left(x_{i+1}\right) \rho_{i+1}^{n}-u\left(x_{i-1}\right) \rho_{i-1}^{n}\right) \quad \text { with } \quad n \geq 1 .
\end{aligned}
$$

Moreover, we have

$$
\left\{\begin{array}{l}
\rho_{i+1}^{n}=\left(g_{1, i+2}^{n-1}+g_{2, i}^{n-1}\right)\left(1-\frac{\eta}{2}\right)+\left(g_{1, i}^{n-1}+g_{2, i+2}^{n-1}\right) \frac{\eta}{2}-\frac{\eta}{2} \cdot \frac{\Delta t}{\Delta x}\left[u\left(x_{i+2}\right) \rho_{i+2}^{n-1}-u\left(x_{i}\right) \rho_{i}^{n-1}\right], \\
\rho_{i-1}^{n}=\left(g_{1, i}^{n-1}+g_{2, i-2}^{n-1}\right)\left(1-\frac{\eta}{2}\right)+\left(g_{1, i-2}^{n-1}+g_{2, i}^{n-1}\right) \frac{\eta}{2}-\frac{\eta}{2} \cdot \frac{\Delta t}{\Delta x}\left[u\left(x_{i}\right) \rho_{i}^{n-1}-u\left(x_{i-2}\right) \rho_{i-2}^{n-1}\right]
\end{array} \quad \text { with } \quad n \geq 1\right.
$$

which allows to obtain (155) by using (156). We conclude the proof as for the $\mathrm{LBM}^{*}$ scheme by noting that

$$
\left\{\begin{array}{l}
g_{1, i}^{0}=\rho_{i}^{0} \cdot\left[(1-\alpha)-\beta \frac{\Delta t}{\Delta x} u\left(x_{i}\right)\right] \\
g_{2, i}^{0}=\rho_{i}^{0} \cdot\left[\alpha+\beta \frac{\Delta t}{\Delta x} u\left(x_{i}\right)\right]
\end{array}\right.
$$


coupled to the LBM scheme (35) implies that

$$
\begin{aligned}
\rho_{i}^{1}= & \left\{\rho_{i+1}^{0} \cdot\left[(1-\alpha)-\beta \frac{\Delta t}{\Delta x} u\left(x_{i+1}\right)\right]+\rho_{i-1}^{0} \cdot\left[\alpha+\beta \frac{\Delta t}{\Delta x} u\left(x_{i-1}\right)\right]\right\}\left(1-\frac{\eta}{2}\right) \\
& +\left\{\rho_{i-1}^{0} \cdot\left[(1-\alpha)-\beta \frac{\Delta t}{\Delta x} u\left(x_{i-1}\right)\right]+\rho_{i+1}^{0} \cdot\left[\alpha+\beta \frac{\Delta t}{\Delta x} u\left(x_{i+1}\right)\right]\right\} \frac{\eta}{2} \\
& -\frac{\eta}{2} \cdot \frac{\Delta t}{\Delta x}\left[u\left(x_{i+1}\right) \rho_{i+1}^{0}-u\left(x_{i-1}\right) \rho_{i-1}^{0}\right] \\
= & {\left[\alpha\left(1-\frac{\eta}{2}\right)+(1-\alpha) \frac{\eta}{2}\right] \rho_{i-1}^{0}+\left[(1-\alpha)\left(1-\frac{\eta}{2}\right)+\alpha \frac{\eta}{2}\right] \rho_{i+1}^{0} } \\
& -\frac{\Delta t}{\Delta x} \rho_{i+1}^{0} u\left(x_{i+1}\right)\left[\beta\left(1-\frac{\eta}{2}\right)-\beta \frac{\eta}{2}+\frac{\eta}{2}\right]+\frac{\Delta t}{\Delta x} \rho_{i-1}^{0} u\left(x_{i-1}\right)\left[\beta\left(1-\frac{\eta}{2}\right)-\beta \frac{\eta}{2}+\frac{\eta}{2}\right]
\end{aligned}
$$

that is to say

$$
\rho_{i}^{1}=\xi \rho_{i-1}^{0}+(1-\xi) \rho_{i+1}^{0}-\gamma \frac{\Delta t}{\Delta x}\left[\rho_{i+1}^{0} u\left(x_{i+1}\right)-\rho_{i-1}^{0} u\left(x_{i-1}\right)\right]
$$

where $\xi=\frac{\eta}{2}+\alpha(1-\eta)$ and $\gamma=\frac{\eta}{2}+\beta(1-\eta)$.

\section{Proof of Lemma 4.2:}

- Study of the LBM* scheme:

To prove Lemma 4.1 in the case of the $\mathrm{LBM}^{*}$ scheme (38), we used (153) and (154) which come from an application of the $\mathrm{LBM}^{*}$ scheme in the cells $i$ and $i \pm 1$. Thus, to obtain the equivalence between the $\mathrm{LBM}^{*}$ scheme (51) (obtained when $u(x)=0$ ) and the Du Fort-Frankel scheme (54) in the cell $i=1$, the LBM scheme has to be applied when $i=0, i=1$ and $i=2$. When $i=2$, we do not have any difficulty to apply the $\mathrm{LBM}^{*}$ scheme (51). Nevertheless, when $i=0$ and $i=1, g_{2,-1}^{n}$ and $g_{2,0}^{n}$ have to be defined. When the boundary conditions are periodic, $g_{2,-1}^{n}$ and $g_{2,0}^{n}$ are defined. But, when the boundary conditions are not periodic, $g_{2,-1}^{n}$ and $g_{2,0}^{n}$ are not defined a priori. We will define these quantities in such a way the discrete Neumann boundary condition

$$
\rho_{i=0}^{n}=\rho_{i=1}^{n}
$$

is satified. Let us apply the $\mathrm{LBM}^{*}$ scheme (51) when $i=0$. We have

$$
\rho_{0}^{n+1}=g_{1,1}^{n}+g_{2,-1}^{n}
$$

which implies by using (157) at the time $t^{n+1}$ that

$$
g_{2,-1}^{n}=\rho_{1}^{n+1}-g_{1,1}^{n} .
$$

But, we have also

$$
g_{2,0}^{n+1}=g_{2,-1}^{n}\left(1-\frac{\eta}{2}\right)+g_{1,1}^{n} \frac{\eta}{2} .
$$

Thus, we have

$$
g_{2,0}^{n+1}=\left(\rho_{1}^{n+1}-\underset{53}{g_{1,1}^{n}}\right)\left(1-\frac{\eta}{2}\right)+g_{1,1}^{n} \frac{\eta}{2} .
$$


Let us now apply the LBM* scheme (51) when $i=1$. We have

$$
\rho_{1}^{n+1}=g_{1,2}^{n}+g_{2,0}^{n} .
$$

This means that

$$
\begin{aligned}
g_{2,0}^{n+1} & =\left(g_{1,2}^{n}+g_{2,0}^{n}-g_{1,1}^{n}\right)\left(1-\frac{\eta}{2}\right)+g_{1,1}^{n} \frac{\eta}{2} \\
& =g_{1,2}^{n}\left(1-\frac{\eta}{2}\right)+g_{2,0}^{n} \frac{\eta}{2}+\left(g_{2,0}^{n}-g_{1,1}^{n}\right)(1-\eta)
\end{aligned}
$$

But, we have also

$$
g_{1,1}^{n+1}=g_{1,2}^{n}\left(1-\frac{\eta}{2}\right)+g_{2,0}^{n} \frac{\eta}{2}
$$

Thus

$$
g_{2,0}^{n+1}=g_{1,1}^{n+1}+\left(g_{2,0}^{n}-g_{1,1}^{n}\right)(1-\eta)
$$

which gives (53)(a). We conclude by noting that (53)(b) is a consequence of (52)(b) and (55).

- Study of the LBM scheme: Let us apply the LBM scheme (50) when $i=0$ and $i=1$. We have

$$
\rho_{0}^{n+1}=\left(g_{1,1}^{n}+g_{2,-1}^{n}\right)\left(1-\frac{\eta}{2}\right)+\left(g_{1,-1}^{n}+g_{2,1}^{n}\right) \frac{\eta}{2}
$$

and

$$
\rho_{1}^{n+1}=\left(g_{1,2}^{n}+g_{2,0}^{n}\right)\left(1-\frac{\eta}{2}\right)+\left(g_{1,0}^{n}+g_{2,2}^{n}\right) \frac{\eta}{2} .
$$

Thus, by taking into account (157) at the time $t^{n+1}$, we obtain

$$
\left(g_{1,1}^{n}+g_{2,-1}^{n}\right)\left(1-\frac{\eta}{2}\right)+\left(g_{1,-1}^{n}+g_{2,1}^{n}\right) \frac{\eta}{2}=\left(g_{1,2}^{n}+g_{2,0}^{n}\right)\left(1-\frac{\eta}{2}\right)+\left(g_{1,0}^{n}+g_{2,2}^{n}\right) \frac{\eta}{2} .
$$

We have also

$$
g_{1,1}^{n+1}=g_{1,2}^{n}\left(1-\frac{\eta}{2}\right)+g_{2,2}^{n} \frac{\eta}{2}
$$

and

$$
g_{2,0}^{n+1}=g_{2,-1}^{n}\left(1-\frac{\eta}{2}\right)+g_{1,-1}^{n} \frac{\eta}{2} .
$$

Thus, we deduce from (158) that

$$
g_{2,0}^{n+1}=g_{1,1}^{n+1}+\left(g_{2,0}^{n}-g_{1,1}^{n}\right)\left(1-\frac{\eta}{2}\right)+\left(g_{1,0}^{n}-g_{2,1}^{n}\right) \frac{\eta}{2}
$$

that is to say

$$
\begin{aligned}
g_{2,0}^{n+1} & =g_{1,1}^{n+1}+\left(g_{2,0}^{n}-g_{1,1}^{n}\right)(1-\eta)+\left[\left(g_{2,0}^{n}-g_{1,1}^{n}\right)+\left(g_{1,0}^{n}-g_{2,1}^{n}\right)\right] \frac{\eta}{2} \\
& =g_{1,1}^{n+1}+\left(g_{2,0}^{n}-g_{1,1}^{n}\right)(1-\eta)+\left(\rho_{0}^{n}-\rho_{1}^{n}\right) \frac{\eta}{2}
\end{aligned}
$$

which gives (57)(b) by taking into account (157). By using (57)(b), we obtain

$$
g_{1,0}^{n+1}+g_{2,0}^{n+1}=g_{1,0}^{n+1}+g_{1,1}^{n+1}+\left(g_{2,0}^{n}-g_{1,1}^{n}\right)(1-\eta) .
$$


Thus, by using (157) at the time $t^{n+1}$, we obtain

$$
g_{1,1}^{n+1}+g_{2,1}^{n+1}=g_{1,0}^{n+1}+g_{1,1}^{n+1}+\left(g_{2,0}^{n}-g_{1,1}^{n}\right)(1-\eta)
$$

which gives (57)(a). Moreover, (57)(c,d) is a consequence of (52) and (55). At last, we obtain that

$$
\rho_{i}^{1}=\xi \rho_{i-1}^{0}+(1-\xi) \rho_{i+1}^{0}
$$

as in the periodic case.

Proof of Lemma 4.3: The proof is similar to the one of Lemma 4.2.

- Study of the LBM* scheme:

Let us apply the $\mathrm{LBM}^{*}$ scheme (51) when $i=0$. We have

$$
\rho_{0}^{n+1}=g_{1,1}^{n}+g_{2,-1}^{n} .
$$

Thus, by applying the boundary condition

$$
\rho_{i=0}^{n}=\rho_{x_{\min }}
$$

at the time $t^{n+1}$, we obtain that

$$
g_{2,-1}^{n}=\rho_{x_{\min }}-g_{1,1}^{n} .
$$

But, we have also

$$
g_{2,0}^{n+1}=g_{2,-1}^{n}\left(1-\frac{\eta}{2}\right)+g_{1,1}^{n} \frac{\eta}{2} .
$$

Thus, we have

$$
g_{2,0}^{n+1}=\left(\rho_{x_{\min }}-g_{1,1}^{n}\right)\left(1-\frac{\eta}{2}\right)+g_{1,1}^{n} \frac{\eta}{2}
$$

that is to say

$$
g_{2,0}^{n+1}=\frac{\rho_{x_{\min }}}{2}+\left(\frac{\rho_{x_{\min }}}{2}-g_{1,1}^{n}\right)(1-\eta)
$$

which gives (63)(a). We conclude the proof as in the periodic case.

- Study of the LBM scheme:

Let us apply the LBM scheme (50) when $i=0$. We have

$$
g_{1,0}^{n+1}=g_{1,1}^{n}\left(1-\frac{\eta}{2}\right)+g_{2,1}^{n} \frac{\eta}{2}
$$

which gives (65)(a). We obtain (65)(b) by appling (159) at the time $t^{n+1}$. We conclude the proof as in the periodic case. 


\section{E. Proof of Propositions 5.1 and 5.2}

\section{E.1. Proof of Proposition 5.1}

To prove Proposition 5.1, we use the following lemma:

\section{Lemma E.1. Let us define}

$$
\forall i:\left\{\begin{array}{l}
\tilde{g}_{1, i}^{0}:=g_{1, i}^{0}-K_{1}, \\
\tilde{g}_{2, i}^{0}:=g_{2, i}^{0}-K_{2}
\end{array}\right.
$$

and let us apply the $L B M^{*}$ scheme (38)(44) with the initial conditions $\left(\tilde{g}_{1, i}^{0}, \tilde{g}_{2, i}^{0}\right)$. Then, we have

$$
\forall i, \forall n \geq 0: \quad \tilde{\rho}_{i}^{n}=\rho_{i}^{n}-K
$$

with $\tilde{\rho}_{i}^{n}:=\tilde{g}_{1, i}^{n}+\tilde{g}_{2, i}^{n}$ and $K:=K_{1}+K_{2}$.

Proof of Lemma E.1: We have by construction

$$
\tilde{\rho}_{i}^{0}=\rho_{i}^{0}-K
$$

Moreover, we have also

$$
\left\{\begin{array}{l}
\tilde{g}_{1, i}^{1}=a \tilde{g}_{1, i+1}^{0}+b \tilde{g}_{2, i-1}^{0} \\
\tilde{g}_{2, i}^{1}=(1-a) \tilde{g}_{1, i+1}^{0}+(1-b) \tilde{g}_{2, i-1}^{0}
\end{array}\right.
$$

with

$$
\left\{\begin{array}{l}
a=1-\frac{\eta}{2}\left(1+\frac{\Delta t}{\Delta x} u_{0}\right), \\
b=\frac{\eta}{2}\left(1-\frac{\Delta t}{\Delta x} u_{0}\right) .
\end{array}\right.
$$

Thus, we can write

$$
\left\{\begin{array}{l}
\tilde{g}_{1, i}^{1}=a g_{1, i+1}^{0}+b g_{2, i-1}^{0}-\left(a K_{1}+b K_{2}\right), \\
\tilde{g}_{2, i}^{1}=(1-a) g_{1, i+1}^{0}+(1-b) g_{2, i-1}^{0}-\left[(1-a) K_{1}+(1-b) K_{2}\right] .
\end{array}\right.
$$

that is to say

$$
\left\{\begin{array}{l}
\tilde{g}_{1, i}^{1}=g_{1, i}^{1}-\left(a K_{1}+b K_{2}\right), \\
\tilde{g}_{2, i}^{1}=g_{2, i}^{1}-K+\left(a K_{1}+b K_{2}\right) .
\end{array}\right.
$$

This allows to obtain

$$
\tilde{\rho}_{i}^{1}=\rho_{i}^{1}-K
$$

We now prove that

$$
\forall i:\left\{\begin{array}{l}
\tilde{\rho}_{i}^{n-1}=\rho_{i}^{n-1}-K \\
\tilde{\rho}_{i}^{n}=\rho_{i}^{n}-K
\end{array}\right.
$$


implies that

$$
\forall i: \quad \tilde{\rho}_{i}^{n+1}=\rho_{i}^{n+1}-K .
$$

We know that $\tilde{\rho}_{i}^{n-1}, \tilde{\rho}_{i}^{n}$ and $\tilde{\rho}_{i}^{n+1}$ are linked through the relation (45) applied to $\tilde{\rho}$, that is to say

$$
\frac{\tilde{\rho}_{i}^{n+1}-\tilde{\rho}_{i}^{n-1}}{2 \Delta t}+\frac{u_{0}}{2 \Delta x}\left(\tilde{\rho}_{i+1}^{n}-\tilde{\rho}_{i-1}^{n}\right)=\frac{v}{\Delta x^{2}}\left(\tilde{\rho}_{i+1}^{n}-\tilde{\rho}_{i}^{n+1}-\tilde{\rho}_{i}^{n-1}+\tilde{\rho}_{i-1}^{n}\right) .
$$

Thus, by using (161), we can write that

$$
\frac{\tilde{\rho}_{i}^{n+1}-\rho_{i}^{n-1}+K}{2 \Delta t}+\frac{u_{0}}{2 \Delta x}\left(\rho_{i+1}^{n}-\rho_{i-1}^{n}\right)=\frac{v}{\Delta x^{2}}\left(\rho_{i+1}^{n}-\rho_{i}^{n+1}-\rho_{i}^{n-1}+\rho_{i-1}^{n}\right)+\frac{v}{\Delta x^{2}}\left(\rho_{i}^{n+1}-\tilde{\rho}_{i}^{n+1}-K\right) .
$$

On the other side, we have also

$$
\frac{\rho_{i}^{n+1}-\rho_{i}^{n-1}}{2 \Delta t}+\frac{u_{0}}{2 \Delta x}\left(\rho_{i+1}^{n}-\rho_{i-1}^{n}\right)=\frac{v}{\Delta x^{2}}\left(\rho_{i+1}^{n}-\rho_{i}^{n+1}-\rho_{i}^{n-1}+\rho_{i-1}^{n}\right)
$$

by using again (45). This allows to write that

$$
\left(\tilde{\rho}_{i}^{n+1}-\rho_{i}^{n+1}+K\right)\left(\frac{1}{2 \Delta t}+\frac{v}{\Delta x^{2}}\right)=0
$$

which proves (162). We conclude by noting that (161) is verified when $n=1$.

\section{Proof of Proposition 5.1:}

- Stability in $L^{\infty}$ of the $L B M^{*}$ scheme with periodic boundary conditions:

Let us define $C:=\frac{C_{d} \Delta x}{v}\left|u_{0}\right| \geq 0$. We have by construction $\Delta t=C \frac{\Delta x}{\left|u_{0}\right|}$. Thus, we have

$$
\frac{\eta}{2}(1-C) \leq \frac{\eta}{2}\left(1+\frac{\Delta t}{\Delta x} u_{0}\right) \leq \frac{\eta}{2}(1+C)
$$

since $\frac{\Delta t}{\Delta x} u_{0}= \pm C$ and $\eta>0$. Moreover, Condition (70)(a) is equivalent to

$$
C \in\left[0, \min \left(1,2 C_{d}\right)\right] .
$$

Since $\frac{\eta}{2}(1 \pm C)=\frac{1 \pm C}{1+2 C_{d}}$, by using (163) and (164), we obtain

$$
0 \leq \frac{\eta}{2}\left(1+\frac{\Delta t}{\Delta x} u_{0}\right) \leq 1
$$

In the same way, we obtain

$$
0 \leq \frac{\eta}{2}\left(1-\frac{\Delta t}{\Delta x} u_{0}\right) \leq 1 .
$$

As a consequence, we can write that $a$ and $b$ defined with (160) verifies

$$
\left\{\begin{array}{c}
0 \leq a \leq 1, \\
0 \leq b \leq 1 . \\
57
\end{array}\right.
$$


Let us now define

$$
\left\{\begin{array}{l}
\tilde{g}_{1, i}^{0}:=g_{1, i}^{0}-K_{1}, \\
\tilde{g}_{2, i}^{0}:=g_{2, i}^{0}-K_{2}
\end{array}\right.
$$

with

$$
\left\{\begin{array}{l}
K_{1}=\left[(1-\alpha)-\beta \frac{\Delta t}{\Delta x} u_{0}\right] \max _{j} \rho_{j}^{0}, \\
K_{2}=\left[\alpha+\beta \frac{\Delta t}{\Delta x} u_{0}\right] \max _{j} \rho_{j}^{0} .
\end{array}\right.
$$

We now suppose that $\alpha \in[0,1]$ and $\beta=0$ or $\beta=\min (1-\alpha, \alpha)$. In these two cases, we have

$$
\left\{\begin{array}{l}
(1-\alpha)-\beta \frac{\Delta t}{\Delta x} u_{0} \geq 0 \\
\alpha+\beta \frac{\Delta t}{\Delta x} u_{0} \geq 0
\end{array}\right.
$$

Thus, since $\left(g_{1, i}^{0}, g_{2, i}^{0}\right)$ is defined with (44), we obtain

$$
\forall i:\left\{\begin{array}{l}
\tilde{g}_{1, i}^{0} \leq 0 \\
\tilde{g}_{2, i}^{0} \leq 0 .
\end{array}\right.
$$

And, by using the fact that

$$
\left\{\begin{array}{l}
\tilde{g}_{1, i}^{n+1}=a \tilde{g}_{1, i+1}^{n}+b \tilde{g}_{2, i-1}^{n}, \\
\tilde{g}_{2, i}^{n+1}=(1-a) \tilde{g}_{1, i+1}^{n}+(1-b) \tilde{g}_{2, i-1}^{n}
\end{array}\right.
$$

and (165), we can write that

$$
\forall i, \forall n \geq 0: \quad\left\{\begin{array}{l}
\tilde{g}_{1, i}^{n} \leq 0 \\
\tilde{g}_{2, i}^{n} \leq 0
\end{array}\right.
$$

which implies that

$$
\forall i, \forall n \geq 0: \quad \tilde{\rho}_{i}^{n} \leq 0 .
$$

On the other side, by using Lemma E.1, we obtain that

$$
\forall i, \forall n \geq 0: \quad \tilde{\rho}_{i}^{n}=\rho_{i}^{n}-K
$$

with $K:=K_{1}+K_{2}=\max _{j} \rho_{j}^{0}$. By using (167), we obtain

$$
\forall i, \forall n \geq 0: \quad \rho_{i}^{n} \leq \max _{j} \rho_{j}^{0}
$$

We obtain

$$
\forall i, \forall n \geq 0: \quad: \quad \rho_{i}^{n} \geq \min _{j} \rho_{j}^{0}
$$

with the same approach. 
- Stability in $L^{\infty}$ of the LFCCDF scheme with periodic boundary conditions:

By using the stability result in $L^{\infty}$ of the $\mathrm{LBM}^{*}$ scheme (38)(44) and by using Lemma 4.1, we obtain the stability in $L^{\infty}$ of the LFCCDF scheme (45)(47) under the condition (70).

- Stability in $L^{\infty}$ of the LBM scheme with periodic boundary conditions:

By using the stability result in $L^{\infty}$ of the LFCCDF scheme (45)(46) and by using again Lemma 4.1, we obtain the stability in $L^{\infty}$ of the LBM scheme (35)(44) under the condition (70).

- Consistency and order of the error with periodic boundary conditions:

When $n \geq 2$ and when $\Delta t=C_{d} \frac{\Delta x^{2}}{v}\left(C_{d} \geq 0\right)$, the LFCCDF scheme (45) is consistent and its consistency error is of order $\Delta x^{2}$ [33]. Let us study the first iterate (47). We have

$$
\begin{aligned}
\rho_{i}^{1} & =\alpha \rho_{i-1}^{0}+(1-\alpha) \rho_{i+1}^{0}-\beta \frac{\Delta t}{\Delta x}\left[\rho_{i+1}^{0} u\left(x_{i+1}\right)-\rho_{i-1}^{0} u\left(x_{i-1}\right)\right] \\
& =\rho_{\text {exact }}\left(0, x_{i}\right)+O\left(\Delta x^{\theta}\right) \\
& =\rho_{\text {exact }}\left(\Delta t, x_{i}\right)+O\left(\Delta t, \Delta x^{\theta}\right)
\end{aligned}
$$

with

$$
\left\{\begin{array}{l}
(\alpha, \beta) \neq(1 / 2,1 / 2) \Longrightarrow \theta=1, \\
(\alpha, \beta)=(1 / 2,1 / 2) \Longrightarrow \theta=2
\end{array}\right.
$$

where $\rho_{\text {exact }}$ is the exact solution of the convection-diffusion equation. Thus, the consistency error is of order $\Delta x$ when $(\alpha, \beta) \neq(1 / 2,1 / 2)$ and is of order $\Delta x^{2}$ when $(\alpha, \beta)=(1 / 2,1 / 2)$.

- Convergence in $L^{\infty}$ with periodic boundary conditions:

We obtain the convergence in $L^{\infty}$ of the LFCCDF scheme by applying the Lax Theorem. Thus, by using again Lemmae 4.1, we also obtain the convergence in $L^{\infty}$ of the LBM and $\mathrm{LBM}^{*}$ schemes.

\section{E.2. Proof of Proposition 5.2}

Firstly, we prove the stability in $L^{\infty}$ for any $C_{d} \geq 0$ of the $\mathrm{LBM}^{*}$ scheme (51). Indeed, it is more simple to analyze this scheme than the LBM scheme (50). Then, by applying Lemmae 4.1, 4.2 and 4.3 (and the Lax Theorem), we easily obtain the other results.

- Stability in $L^{\infty}$ of the $L B M^{*}$ scheme with periodic boundary conditions:

Since $\eta \in] 0,2]$, we deduce from (51) that

$$
\max _{i}\left(\left|g_{1, i}^{n+1}\right|,\left|g_{2, i}^{n+1}\right|\right) \leq \max _{i}\left(\left|g_{1, i}^{n}\right|,\left|g_{2, i}^{n}\right|\right)
$$


which proves the unconditionnal stability in $L^{\infty}$ as soon as the initial condition is bounded. Moreover, since $\rho_{i}^{n}=g_{1, i}^{n}+g_{2, i}^{n}$, we have

$$
\max _{i}\left|\rho_{i}^{n}\right| \leq 2 \max _{i}\left(\left|g_{1, i}^{n}\right|,\left|g_{2, i}^{n}\right|\right)
$$

Thus, we deduce from (168) that

$$
\max _{i}\left|\rho_{i}^{n}\right| \leq 2 \max _{i}\left(\left|g_{1, i}^{0}\right|,\left|g_{2, i}^{0}\right|\right)
$$

that is to say

$$
\max _{i}\left|\rho_{i}^{n}\right| \leq 2 \max (|1-\alpha|,|\alpha|) \cdot \max _{i}\left|\rho_{i}^{0}\right|
$$

by using the initial condition (52).

- Stability in $L^{\infty}$ of the $L B M^{*}$ scheme with Neumann boundary conditions:

Since $\eta \in] 0,2]$, we deduce from (51) that

$$
\max _{i \geq 1}\left(\left|g_{1, i}^{n+1}\right|,\left|g_{2, i}^{n+1}\right|\right) \leq \max \left[\left|g_{2,0}^{n}\right|, \max _{i \geq 1}\left(\left|g_{1, i}^{n}\right|,\left|g_{2, i}^{n}\right|\right)\right] .
$$

Inequalities (168) and (170) are different because of the boundary term $\left|g_{2,0}^{n}\right|$ in (170) which does not exist when the boundary conditions are periodic. The difficulty to obtain the stability in $L^{\infty}$ comes from this term. We deduce from the boundary condition (53)(a) that

$$
\begin{aligned}
g_{2,0}^{n+1} & =g_{1,1}^{n+1}+(1-\eta) g_{2,0}^{n}-(1-\eta) g_{1,1}^{n} \\
& =g_{1,1}^{n+1}+(1-\eta)\left[g_{1,1}^{n}+(1-\eta) g_{2,0}^{n-1}-(1-\eta) g_{1,1}^{n-1}\right]-(1-\eta) g_{1,1}^{n} \\
& =g_{1,1}^{n+1}+(1-\eta)^{2} g_{2,0}^{n-1}-(1-\eta)^{2} g_{1,1}^{n-1} \\
& =\cdots \\
& =g_{1,1}^{n+1}+(1-\eta)^{n+1} g_{2,0}^{0}-(1-\eta)^{n+1} g_{1,1}^{0}
\end{aligned}
$$

that is to say

$$
g_{2,0}^{n+1}=g_{1,1}^{n+1}+(1-\eta)^{n+1}\left(g_{2,0}^{0}-g_{1,1}^{0}\right) .
$$

On the other side, we have

$$
g_{1,1}^{n+1}=g_{1,2}^{n}\left(1-\frac{\eta}{2}\right)+g_{2,0}^{n} \frac{\eta}{2}
$$

Thus, by using (171), we obtain

$$
g_{2,0}^{n+1} \leq \max \left(\left|g_{1,2}^{n}\right|,\left|g_{2,0}^{n}\right|\right)+|1-\eta|^{n+1} \cdot\left|g_{2,0}^{0}-g_{1,1}^{0}\right| .
$$

By injecting this inequality in (170), we find

$$
\max \left[\left|g_{2,0}^{n+1}\right|, \max _{i \geq 1}\left(\left|g_{1, i}^{n+1}\right|,\left|g_{2, i}^{n+1}\right|\right)\right] \leq \max \left[\max \left(\left|g_{1,2}^{n}\right|,\left|g_{2,0}^{n}\right|\right)+|1-\eta|^{n+1} \cdot\left|g_{2,0}^{0}-g_{1,1}^{0}\right|,\left|g_{2,0}^{n}\right|, \max _{i \geq 1}\left(\left|g_{1, i}^{n}\right|,\left|g_{2, i}^{n}\right|\right)\right] .
$$

Let us now define

$$
G^{n}:=\max \left[\left|g_{2,0}^{n}\right|, \max _{i \geq 1}\left(\left|g_{1, i}^{n}\right|,\left|g_{2, i}^{n}\right|\right)\right]
$$


We deduce from (172) that

$$
G^{n+1} \leq G^{n}+|1-\eta|^{n+1} \cdot\left|g_{2,0}^{0}-g_{1,1}^{0}\right|
$$

that is to say

$$
G^{n+1} \leq G^{0}+|1-\eta| S_{n} \cdot\left|g_{2,0}^{0}-g_{1,1}^{0}\right|
$$

where

$$
S_{n}:=\sum_{k=0}^{n}|1-\eta|^{k}
$$

Let us now suppose that $\eta \in] 0,2\left[\right.$ that is to say $C_{d}>0$. By noting that $S_{n} \leq \frac{1}{1-|\eta-1|}$ when $\left.\eta \in\right] 0,2[$, we obtain

$$
G^{n+1} \leq G^{0}+\frac{|1-\eta|}{1-|1-\eta|}\left|g_{2,0}^{0}-g_{1,1}^{0}\right|
$$

which proves the unconditionnal stability in $L^{\infty}$ as soon as the initial condition is bounded. Moreover, by applying the arguments used to obtain (169) in the periodic case, we deduce from (176) that

$$
\max _{i \geq 0}\left|\rho_{i}^{n+1}\right| \leq 2 \max (|1-\alpha|,|\alpha|) \cdot \max _{i \geq 0}\left|\rho_{i}^{0}\right|+2|2 \alpha-1| \frac{|1-\eta|}{1-|1-\eta|}\left|\rho_{1}^{0}\right|
$$

that is to say

$$
\max _{i \geq 1}\left|\rho_{i}^{n+1}\right| \leq 2 \max (|1-\alpha|,|\alpha|) \cdot \max _{i \geq 1}\left|\rho_{i}^{0}\right|+2|2 \alpha-1| \frac{|1-\eta|}{1-|1-\eta|}\left|\rho_{1}^{0}\right|
$$

when $\eta \in] 0,2\left[\right.$ since $\rho_{0}^{n}=\rho_{1}^{n}$. When $\eta=2$ that is to say $C_{d}=0$, we obtain

$$
\max _{i \geq 1}\left|\rho_{i}^{n+1}\right| \leq 2 \max (|1-\alpha|,|\alpha|) \cdot \max _{i \geq 1}\left|\rho_{i}^{0}\right|
$$

by using Lemma 7.2. At last, when $\alpha=\frac{1}{2}$ and for any $\left.\left.\eta \in\right] 0,2\right]$, we have $g_{2,0}^{0}=g_{1,1}^{0}$ which implies that $G^{n+1} \leq G^{0}$ by using (174). Thus, (177) is also astisfied.

- Stability in $L^{\infty}$ of the LBM $^{*}$ scheme with Dirichlet boundary conditions:

Inequality (170) is still satisfied. Moreover, we deduce from the boundary condition (63)(a) that

$$
\left|g_{2,0}^{n+1}\right| \leq\left(1-\frac{\eta}{2}\right)\left|\rho_{x_{\min }}\right|+|\eta-1| \cdot\left|g_{1,1}^{n}\right|
$$

Thus, by using (170), we obtain

$$
\max \left[\left|g_{2,0}^{n+1}\right|, \max _{i \geq 1}\left(\left|g_{1, i}^{n+1}\right|,\left|g_{2, i}^{n+1}\right|\right)\right] \leq \max \left[\left(1-\frac{\eta}{2}\right)\left|\rho_{x_{\min }}\right|+|\eta-1| \cdot\left|g_{1,1}^{n}\right|,\left|g_{2,0}^{n}\right|, \max _{i \geq 1}\left(\left|g_{1, i}^{n}\right|,\left|g_{2, i}^{n}\right|\right)\right] .
$$

We deduce from (178) that

$$
G^{n+1} \leq \max \left[\left(1-\frac{\eta}{2}\right)\left|\rho_{x_{\min }}\right|+|\eta-1| G^{n}, G^{n}\right] .
$$

where $G^{n}$ is defined with (173). Thus, we have also

$G^{n+1} \leq \max \left[\left(1-\frac{\eta}{2}\right)\left|\rho_{x_{\min }}\right|+|\eta-1| \max \left[\left(1-\frac{\eta}{2}\right)\left|\rho_{x_{\min }}\right|+|\eta-1| G^{n-1}, G^{n-1}\right],\left(1-\frac{\eta}{2}\right)\left|\rho_{x_{\min }}\right|+|\eta-1| G^{n-1}, G^{n-1}\right]$ 
that is to say

$$
G^{n+1} \leq \max \left[\left(1-\frac{\eta}{2}\right)\left|\rho_{x_{\min }}\right| \cdot(1+|\eta-1|)+|\eta-1|^{2} G^{n-1},\left(1-\frac{\eta}{2}\right)\left|\rho_{x_{\min }}\right|+|\eta-1| G^{n-1}, G^{n-1}\right] .
$$

The previous inequalities incite us to prove that

$$
\begin{aligned}
G^{n+1} \leq \max & {\left[\left(1-\frac{\eta}{2}\right)\left|\rho_{x_{\min }}\right| S_{m}+|\eta-1|^{m+1} G^{n-m},\right.} \\
& \left(1-\frac{\eta}{2}\right)\left|\rho_{x_{\min }}\right| S_{m-1}+|\eta-1|^{m} G^{n-m}, \ldots, \\
& \left.\left(1-\frac{\eta}{2}\right)\left|\rho_{x_{\min }}\right| S_{0}+|\eta-1| G^{n-m}, G^{n-m}\right]
\end{aligned}
$$

where $S_{m}$ is defined with (175). We know that (180) is verified when $m=0$ and $m=1$. Let us now suppose that (180) is verified at the rank $m$. By injecting (179) in (180), we obtain

$$
\begin{aligned}
G^{n+1} \leq \max & \left\{\left(1-\frac{\eta}{2}\right)\left|\rho_{x_{\min }}\right| S_{m}+|\eta-1|^{m+1} \max \left[\left(1-\frac{\eta}{2}\right)\left|\rho_{x_{\min }}\right|+|\eta-1| G^{n-m-1}, G^{n-m-1}\right],\right. \\
& \left(1-\frac{\eta}{2}\right)\left|\rho_{x_{\min }}\right| S_{m-1}+|\eta-1|^{m} \max \left[\left(1-\frac{\eta}{2}\right)\left|\rho_{x_{\min }}\right|+|\eta-1| G^{n-m-1}, G^{n-m-1}\right], \ldots, \\
& \left(1-\frac{\eta}{2}\right)\left|\rho_{x_{\min }}\right| S_{0}+|\eta-1| \max \left[\left(1-\frac{\eta}{2}\right)\left|\rho_{x_{\min }}\right|+|\eta-1| G^{n-m-1}, G^{n-m-1}\right], \\
& \left.\max \left[\left(1-\frac{\eta}{2}\right)\left|\rho_{x_{\min }}\right|+|\eta-1| G^{n-m-1}, G^{n-m-1}\right]\right\}
\end{aligned}
$$

which gives

$$
\begin{aligned}
G^{n+1} \leq \max & {\left[\left(1-\frac{\eta}{2}\right)\left|\rho_{x_{\min }}\right|\left(S_{m}+|\eta-1|^{m+1}\right)+|\eta-1|^{m+2} G^{n-m-1},\left(1-\frac{\eta}{2}\right)\left|\rho_{x_{\min }}\right| S_{m}+|\eta-1|^{m+1} G^{n-m-1}\right.} \\
& \left(1-\frac{\eta}{2}\right)\left|\rho_{x_{\min }}\right|\left(S_{m-1}+|\eta-1|^{m}\right)+|\eta-1|^{m+1} G^{n-m-1},\left(1-\frac{\eta}{2}\right)\left|\rho_{x_{\min }}\right| S_{m-1}+|\eta-1|^{m} G^{n-m-1}, \ldots, \\
& \left(1-\frac{\eta}{2}\right)\left|\rho_{x_{\min }}\right|\left(S_{0}+|\eta-1|\right)+|\eta-1|^{2} G^{n-m-1},\left(1-\frac{\eta}{2}\right)\left|\rho_{x_{\min }}\right| S_{0}+|\eta-1| G^{n-m-1} \\
& \left.\left(1-\frac{\eta}{2}\right)\left|\rho_{x_{\min }}\right|+|\eta-1| G^{n-m-1}, G^{n-m-1}\right]
\end{aligned}
$$

that is to say

$$
\begin{aligned}
G^{n+1} \leq \max & {\left[\left(1-\frac{\eta}{2}\right)\left|\rho_{x_{\min }}\right| S_{m+1}+|\eta-1|^{m+2} G^{n-m-1},\right.} \\
& \left(1-\frac{\eta}{2}\right)\left|\rho_{x_{\min }}\right| S_{m}+|\eta-1|^{m+1} G^{n-m-1}, \ldots, \\
& \left.\left(1-\frac{\eta}{2}\right)\left|\rho_{x_{\min }}\right| S_{0}+|\eta-1| G^{n-m-1}, G^{n-m-1}\right] .
\end{aligned}
$$


Thus, (180) is also verified at the rank $m+1$, which proves (180) for any $m \in\{0, \ldots, n-1\}$. Let us now suppose that $\eta \in] 0,2\left[\right.$ that is to say $C_{d}>0$. By applying (180) at the rank $n-1$, by noting that $S_{m} \leq \frac{1}{1-|\eta-1|}$ and that $|\eta-1|^{m} \leq 1$ for any $m \in \mathbb{N}$, we obtain that

$$
G^{n+1} \leq \frac{1-\frac{\eta}{2}}{1-|\eta-1|}\left|\rho_{x_{\min }}\right|+G^{0}
$$

which proves the unconditionnal stability in $L^{\infty}$ as soon as the initial condition is bounded. Moreover, by applying the arguments used to obtain (169) in the periodic case, we deduce from (181) that

$$
\max _{i \geq 0}\left|\rho_{i}^{n+1}\right| \leq 2 \max (|1-\alpha|,|\alpha|) \max _{i \geq 0}\left|\rho_{i}^{0}\right|+\frac{2\left(1-\frac{\eta}{2}\right)}{1-|\eta-1|}\left|\rho_{x_{\min }}\right|
$$

that is to say

$$
\max _{i \geq 1}\left|\rho_{i}^{n+1}\right| \leq 2 \max (|1-\alpha|,|\alpha|) \cdot \max \left(\max _{i \geq 1}\left|\rho_{i}^{0}\right|,\left|\rho_{x_{\min }}\right|\right)+\frac{2\left(1-\frac{\eta}{2}\right)}{1-|\eta-1|}\left|\rho_{x_{\min }}\right|
$$

since $\rho_{i}^{n}=\rho_{x_{\min }}$. When $\eta=2$ that is to say $C_{d}=0$, we obtain (177) by using Lemma 7.2.

- We prove the other statements as in the proof of Proposition 5.1 (that is to say by using Lemmae 4.1, 4.2 and 4.3, and the Lax Theorem).

\section{F. Proof of Propositions 6.1 and 6.2, and of Lemma 6.1}

\section{Proof of Proposition 6.1:}

We focus on the $\mathrm{LBM}^{*}$ scheme (51) since this scheme is more simple than the LBM scheme (50). Then, by applying Lemmae 4.1 and 4.2, we obtain the results for the Du Fort-Frankel scheme and, then, for the LBM scheme (50) (this approach was also used for the proof of Proposition 5.2).

- Discrete Maximum principle with periodic boundary conditions for the $L B M^{*}$ scheme when $\alpha=\frac{1}{2}$ :

The $\mathrm{LBM}^{*}$ scheme (51) implies that

$$
\min _{j}\left(g_{1, j}^{n}, g_{2, j}^{n}\right) \leq g_{q, i}^{n+1} \leq \max _{j}\left(g_{1, j}^{n}, g_{2, j}^{n}\right) \quad(q \in\{1,2\})
$$

since $\eta \in] 0,2]$.Thus, we have

$$
\min _{j}\left(g_{1, j}^{0}, g_{2, j}^{0}\right) \leq g_{q, i}^{n+1} \leq \max _{j}\left(g_{1, j}^{0}, g_{2, j}^{0}\right) \quad(q \in\{1,2\}) .
$$

Thus, by using (52),we obtain

$$
\min [(1-\alpha), \alpha] \cdot \min _{j} \rho_{j}^{0} \leq g_{q, i}^{n} \leq \max [(1-\alpha), \alpha] \cdot \max _{j} \rho_{j}^{0} \quad(q \in\{1,2\})
$$


when $\alpha \in[0,1]$. Since $\rho_{i}^{n}=g_{1, i}^{n}+g_{2, i}^{n}$, we deduce from (183) that

$$
2 \min [(1-\alpha), \alpha] \cdot \min _{j} \rho_{j}^{0} \leq \rho_{i}^{n} \leq 2 \max [(1-\alpha), \alpha] \cdot \max _{j} \rho_{j}^{0} .
$$

Thus, we deduce from (184) that the discrete maximum principle (74) is verified when $\alpha=\frac{1}{2}$.

- Discrete Maximum principle with periodic boundary conditions for the $L_{B M}^{*}$ scheme when $\alpha \in[0,1]$ :

The discrete maximum principle (74) cannnot be deduced from (184) when $\alpha \neq \frac{1}{2}$. Nevertheless, we now prove that (74) is still satisfied when $\alpha \in[0,1]$. To obtain this result, we prove that

$$
\left\{\begin{array}{l}
g_{1, i}^{n}=\sum_{k} \Gamma_{k}^{n} g_{1, i_{k}^{1}}^{0}+\sum_{k} \widetilde{\Gamma}_{k}^{n} g_{2, i_{k}^{2}}^{0}, \\
g_{2, i}^{n}=\sum_{k} \widetilde{\Gamma}_{k}^{n} g_{1, i_{k}^{1}}^{0}+\sum_{k} \Gamma_{k}^{n} g_{2, i_{k}^{2}}^{0}, \\
\sum_{k}\left(\Gamma_{k}^{n}+\widetilde{\Gamma}_{k}^{n}\right)=1, \\
\Gamma_{k}^{n} \geq 0 \\
\widetilde{\Gamma}_{k} \geq 0
\end{array}\right.
$$

where $\left\{i_{k}^{1}\right\}_{k}$ et $\left\{i_{k}^{2}\right\}_{k}$ are two sequences which depend on $i$, and where $\left\{\Gamma_{k}^{n}\right\}_{k}$ and $\left\{\widetilde{\Gamma}_{k}^{n}\right\}_{k}$ are two positive real sequences. It is obvious that (185) is verified when $n=1$ since

$$
\left\{\begin{array}{l}
g_{1, i}^{1}=g_{1, i+1}^{0}\left(1-\frac{\eta}{2}\right)+g_{2, i-1}^{0} \frac{\eta}{2}, \\
g_{2, i}^{1}=g_{2, i-1}^{0}\left(1-\frac{\eta}{2}\right)+g_{1, i+1}^{0} \frac{\eta}{2} .
\end{array}\right.
$$

Let us suppose that (185) is satisfied at the rank $n$. Then, the $\mathrm{LBM}^{*}$ scheme (51) can be written with

$$
\left\{\begin{array}{l}
g_{1, i}^{n+1}=\left(1-\frac{\eta}{2}\right)\left(\sum_{k} \Gamma_{k}^{n} g_{1, i_{k}^{1}+1}^{0}+\sum_{k} \widetilde{\Gamma}_{k}^{n} g_{2, i_{k}^{2}+1}^{0}\right)+\frac{\eta}{2}\left(\sum_{k} \widetilde{\Gamma}_{k}^{n} g_{1, i_{k}^{1}-1}^{0}+\sum_{k} \Gamma_{k}^{n} g_{2, i_{k}^{2}-1}^{0}\right), \\
g_{2, i}^{n+1}=\frac{\eta}{2}\left(\sum_{k} \Gamma_{k}^{n} g_{1, i_{k}+1}^{0}+\sum_{k} \widetilde{\Gamma}_{k}^{n} g_{2, i_{k}^{2}+1}^{0}\right)+\left(1-\frac{\eta}{2}\right)\left(\sum_{k} \widetilde{\Gamma}_{k}^{n} g_{1, i_{k}^{1}-1}^{0}+\sum_{k} \Gamma_{k}^{n} g_{2, i_{k}^{2}-1}^{0}\right)
\end{array}\right.
$$

that is to say with

$$
\left\{\begin{array}{c}
g_{1, i}^{n+1}=\sum_{k}\left[\left(1-\frac{\eta}{2}\right) \Gamma_{k}^{n} g_{1, i_{k}^{1}+1}^{0}+\frac{\eta}{2} \widetilde{\Gamma}_{k}^{n} g_{1, i_{k}^{1}-1}^{0}\right]+\sum_{k}\left[\left(1-\frac{\eta}{2}\right) \widetilde{\Gamma}_{k}^{n} g_{2, i_{k}^{2}+1}^{0}+\frac{\eta}{2} \Gamma_{k}^{n} g_{2, i_{k}^{2}-1}^{0}\right] \\
g_{2, i}^{n+1}=\sum_{k}\left[\frac{\eta}{2} \Gamma_{k}^{n} g_{1, i_{k}^{1}+1}^{0}+\left(1-\frac{\eta}{2}\right) \widetilde{\Gamma}_{k}^{n} g_{1, i_{k}^{1}-1}^{0}\right]+\sum_{k}\left[\frac{\eta}{2} \widetilde{\Gamma}_{k}^{n} g_{2, i_{k}^{2}+1}^{0}+\left(1-\frac{\eta}{2}\right) \Gamma_{k}^{n} g_{2, i_{k}^{2}-1}^{0}\right] \\
64
\end{array}\right.
$$


But, (186) can be written with (185)(a,b) at the rank $n+1$ after a reorganization of the sequences. Moreover, we have

$$
\sum_{k}\left[\left(1-\frac{\eta}{2}\right) \Gamma_{k}^{n}+\frac{\eta}{2} \widetilde{\Gamma}_{k}^{n}\right]+\sum_{k}\left[\frac{\eta}{2} \Gamma_{k}^{n}+\left(1-\frac{\eta}{2}\right) \widetilde{\Gamma}_{k}^{n}\right]=\sum_{k}\left(\Gamma_{k}^{n}+\widetilde{\Gamma}_{k}^{n}\right)=1
$$

and

$$
\left\{\begin{array}{l}
\left(1-\frac{\eta}{2}\right) \Gamma_{k}^{n} \geq 0 \\
\frac{\eta}{2} \widetilde{\Gamma}_{k}^{n} \geq 0 \\
\frac{\eta}{2} \Gamma_{k}^{n} \geq 0 \\
\left(1-\frac{\eta}{2}\right) \widetilde{\Gamma}_{k}^{n} \geq 0
\end{array}\right.
$$

since $\eta \in] 0,2]$. Thus, (185) is satisfied for any $n \geq 1$. By using the fact that $\rho_{i}^{n}=g_{1, i}^{n}+g_{2, i}^{n}$ and by using (52), (185)(a,b) implies that

$$
\rho_{i}^{n}=\sum_{k}\left(\Gamma_{k}^{n}+\widetilde{\Gamma}_{k}^{n}\right)\left(g_{1, i_{k}^{1}}^{0}+g_{2, i_{k}}^{0}\right)=\sum_{k}\left(\Gamma_{k}^{n}+\widetilde{\Gamma}_{k}^{n}\right)\left[(1-\alpha) \rho_{i_{k}^{1}}^{0}+\alpha \rho_{i_{k}^{2}}^{0}\right]
$$

since $\rho_{i}^{n}=g_{1, i}^{n}+g_{2, i}^{n}$. Thus, because of $(185)(\mathrm{c}, \mathrm{d}, \mathrm{e})$, we obtain that $\rho_{i}^{n}$ is a convex combination of $\left\{\rho_{j}^{0}\right\}_{j}$ when $\alpha \in[0,1]$ which allows to obtain (74).

- Discrete Maximum principle with Neumann boundary conditions for the LBM* scheme when $\alpha=\frac{1}{2}$ :

A priori, the proof in the periodic case when $\alpha \in[0,1]$ is not valid in the Neumann case because of the boundary conditions (53) in $x=x_{\min }$. Nevertheless, when $\alpha=\frac{1}{2}$, the boundary conditions (53) are given by

$$
\forall n \geq 0: \quad g_{2,0}^{n}=g_{1,1}^{n}
$$

since $\alpha=\frac{1}{2} \Longrightarrow g_{1,1}^{0}=g_{2,1}^{0}=\frac{\rho_{1}^{0}}{2}$ and $g_{2,0}^{0}=\frac{\rho_{1}^{0}}{2}$ that is to say $g_{2,0}^{0}=g_{1,1}^{0}$. As a consequence, the proof in the periodic case with $\alpha=\frac{1}{2}$ becomes valid in the Neumann case.

Proof of Lemma 6.1: In the Dirichlet case, the bounday conditions (63) in $x=x_{\min }$ can be rewritten with

$$
\left\{\begin{array}{l}
g_{2, i=0}^{n+1}=\frac{\rho_{x_{\min }}}{2}(2-\eta)+g_{1, i=1}^{n}(\eta-1), \\
g_{2, i=0}^{n=0}=\alpha \rho_{x_{\min }} .
\end{array}\right.
$$

We deduce from (188) that when $\eta \in[1,2]$ that is to say when $C_{d} \in\left[0, \frac{1}{2}\right]$, we have

$$
\min \left[\frac{\rho_{x_{\min }}}{2}, g_{1, i=1}^{n}\right] \leq g_{2, i=0}^{n+1} \leq \max \left[\frac{\rho_{x_{\min }}}{2}, g_{1, i=1}^{n}\right] \text {. }
$$

Thus, the proof in the periodic case with $\alpha=\frac{1}{2}$ can be applied. $\square$ 
Proof of Proposition 6.2: The proof is identical to the periodic case with $\alpha=\frac{1}{2}$ by replacing (182) with

$$
\min \left[\frac{\rho_{x_{\min }}}{2}, \min _{j \geq 1}\left(g_{1, j}^{n}, g_{2, j}^{n}\right)\right] \leq g_{1, i}^{n+1} \leq \max \left[\frac{\rho_{x_{\min }}}{2}, \max _{j \geq 1}\left(g_{1, j}^{n}, g_{2, j}^{n}\right)\right]
$$

\section{References}

[1] U. Frisch, B. Hasslacher, Y. Pomeau, Lattice-Gas Automata for the Navier-Stokes Equation, Phys. R. Letters 56 (14) (1986) 1505-1508. 1

[2] D. d'Humières, P. Lallemand, Ecoulement d'un gaz sur réseau dans un canal bidimensionnel : développement du profil de Poiseuille, C. R. Acad. Sc. Paris (Série II) 302 (16) (1986) 983-988. 1

[3] G. McNamara, G. Zanetti, Use of a Boltzmann Equation to Simulate Lattice-Gas Automata, Phys. R. Letters 61 (20) (1988) 2332-2335. 1

[4] D. Wolf-Gladrow, A Lattice Bolzmann Equation for Diffusion, J. of Stat. Phys. 79 (5,6) (1995) 1023-1032. 1, 2,10

[5] S. Suga, An Accurate Multi-level Finite Difference Scheme for 1D Diffusion Equations Derived from the Lattice Boltzmann Method, J. Stat. Phys. 140 (2010) 494-503. 1, 3

[6] G. Kaluza, The numerical solution of transient heat conduction problem using the lattice Bolzmann method, Scientific Research of the Institute of Mathematics and Computer Science 1 (11) (2012) 23-30. 1, 2, 10, 16,17

[7] W. Jiaung, J. Ho, C. Kuo, Lattice Bolzmann Method for the Heat Conduction Problem with Phase Change, Numerical Heat Transfer, Part B 39 (2001) 167-187. 1, 2, 10

[8] S. Mishra, A. Lankadasu, K. Beronov, Application of the Lattice Boltzmann Method for Solving the Energy Equation of a 2-D Transcient Condution-Radiation Problem, Int. J. of Heat and Mass Transfer 48 (2005) 3648-3659. 1

[9] J. Ho, C. Kuo, W. Jiaung, C. Twu, Lattice Bolzmann Scheme for Hyperbolic Heat Conduction Equation, Numerical Heat Transfer, Part B 41 (2002) 591-607. 1

[10] I. Ginzburg, Variably Saturated Flow Described with Anisotropic Lattice Boltzmann Methods, Computer and Fluids 35 (2006) 831-848. 1

[11] S. Suga, Numerical schemes obtained from lattice Boltzmann equations for advection diffusion equations, Int. J. of Mod. Phys. C 17 (11) (2006) 1563-1577. 1, 3

[12] S. Suga, Stability and accuracy of lattice Boltzmann schemes for anisotropic advection-diffusion equations, Int. J. of Mod. Phys. C 20 (4) (2009) 633-650. 1, 3

[13] B. Servan-Camas, F. T.-C. Tsai, Lattice Boltzmann method with two relaxation times for advection-diffusion equation: Third order analysis and stability analysis, Advances in Water Resources 31 (2008) 1113-1126. 1, 3

[14] I. Ginzburg, Equilibrium-type and Link-type Lattice Boltzmann Models for Generic Advection and Anisotropic-Dispersion Equation, Advances in Water Resources 28 (2005) 1171-1195. 1

[15] I. Ginzburg, D. d'Humières, A. Kuzmin, Optimal Stability of Advection-Diffusion Lattice Boltzmann Models with Two Relaxation Times for Positive/Negative Equilibrium, J. Stat. Phys. 139 (2010) 1090-1143. 1, 3

[16] I. Ginzburg, Truncation Errors, Exact and Heuristic Stability Analysis of Two-Relaxation-Times Lattice Boltzmann Schemes for Anisotropic Advection-Diffusion Equation, Commun. Comput. Phys. 11 (5) (2012) 1439-1502. 1, 3

[17] A. Kuzmin, I. Ginzburg, A. Mohamad, The role of the kinetic parameter in the stability of two-relaxation-time advectiondiffusion lattice Boltzmann schemes, Computers and Mathematics with Applications 61 (2011) 3417-3442. 1, 3

[18] M. Rheinländer, Stability and multiscale analysis of an advective lattice Boltzmann scheme, Progress in Computational Fluid Dynamics 8 (2008) 56-68. 1, 3, 10, 27, 28, 38

[19] R. Nourgaliev, T. Dinh, T. Theofanous, D. Joseph, The Lattice Boltzmann Equation: Theoretical Interpretation, Numerics and Implications, Int. J. of Mult. Flow 29 (2003) 117-169. 1

[20] M. Yoshino, T. Inamuro, Lattice Boltzmann Simulations for Flow and Heat/Mass Transfer Problems in a Three-dimensional Porous Structure, Int. J. Numer. Meth. Fluids 43 (2003) 183-198. 1

[21] H. Zheng, C. Shu, Y. Chew, A Lattice Boltzmann Model for Multiphase Flows with Large Density Ratio, J. Comp. Phys. 218 (1) (2006) 353-371. 1

[22] G. Thömmes, J. Becker, M. Junk, A. Vaikuntam, D. Kehrwald, A. Klar, K. Steiner, A. Wiegmann, A lattice Boltzmann Method for Immiscible Multiphase Flow Simulations using the Level Set Method, J. Comp. Phys. 228 (4) (2009) 1139-1156. 1

[23] I. Ginzburg, K. Steiner, Lattice Boltzmann Model for Free-Surface Flow and its Application to Filling Process in Casting, J. Comp. Phys. 185 (2003) 61-99. 1, 2 
[24] I. Ginzburg, K. Steiner, A Free-Surface Lattice Boltzmann Method for Modelling the Filling of Expanding Cavities by Bingham Fluids, Phil. Trans. R. Soc. Lond. A 360 (2002) 453-466. 1

[25] I. Karlin, S. Ansumali, C. Frouzakis, Elements of the Lattice Boltzmann Method I: Linear advection Equation, Comm. In Comp. Phys. 1 (4) (2006) 616-655. 2, 7

[26] I. Karlin, S. Chikatamarla, S. Ansumali, Elements of the Lattice Boltzmann Method II: Kinetics and hydrodynamics in one dimension, Comm. In Comp. Phys. 1 (1) (2006) 1-44. 2

[27] F. Dubois, Third order Equivalent Equation of Lattice Boltzmann Scheme, Disc. and Cont. Dyn. Syst. 23 (2009) 221-248. 2

[28] F. Dubois, P. Lallemand, Towards Higher Order Lattice Boltzmann Schemes, J. Stat. Mech.: Theory and Experiment P06006. 2

[29] M. Junk, A. Klar, L. Luo, Asymptotic Analysis of the Lattice Boltzmann Equation, J. Comp. Phys. 210 (2) (2005) 676-704. $2,4,13$

[30] M. Junk, Z. Yang, Convergence of Lattice Boltzmann Methods for Navier-Stokes Flows in Periodic and Bounded Domains, Numer. Math. 112 (2009) 65-87. 2

[31] M. Junk, W. Yong, Weighted $L^{2}$-Stability of the Lattice Boltzmann Method, SIAM J. Numer. Anal. 47 (2009) 1651-1665. 2

[32] M. Ancona, Fully-Lagrangian and Lattice-Boltzmann Methods for Solving Systems of Conservation Equations, J. Comp. Phys. 115 (1994) 107-120. 2, 3, 10, 14

[33] Y.-K. Kwok, K.-K. Tam, Stability analysis of three-level difference schemes for initial-boundary problems for multidimensional convective-diffusion equations, Comm. in Num. Meth. in Eng. 9 (1993) 595-605. 3, 14, 19, 59

[34] D. d'Humières, I. Ginzburg, Viscosity independent numerical errors for Lattice Boltzmann models: From recurrence equations to "magic" collision numbers, Computers and Mathematics with Applications 58 (2009) 823-840. 3

[35] E. D. Fort, S. Frankel, Stability Conditions in the Numerical Treatment of Parabolic Differential Equations, Math. Tables Other Aids Comput. 7 (1953) 135-152. 3, 14, 25, 38

[36] S. Jin, C. Levermore, Numerical Schemes for Hyperbolic Conservation Laws with Stiff Relaxation Terms, J. Comp. Phys. 126 (2) (1996) 449-467. 6

[37] X. He, S. Chen, G. Doolen, A Novel Thermal Model for the Lattice Boltzmann Method in Incompressible Limit, J. Comp. Phys. 146 (1998) 282-300. 7

[38] R. Maier, R. Bernard, D. Grunau, Boundary conditions for the lattice Boltzmann method, Phys. Fluids 8 (7) (1996) 17881801. 16

[39] I. Ginzbourg, P. Adler, Boundary flow condition analysis for the three-dimensional lattice Boltzmann model, J. Phys. II France 4 (1994) 191-214. 16

[40] R. Cornubert, D. D’Humières, D. Levermore, A Knudsen layer theory for lattice gases, Physica D 47 (1991) 241-259. 16

[41] S. Goldstein, On Diffusion by Discontinuous movements, and on the Telegraph Equation, The Quarterly Jnl. of Mechanics \& App. Maths 4 (2) (1951) 129-156. 3, 30, 33

[42] J. Banasiak, J. Mika, Singularity Perturbed Telegraph Equations with Applications in the Random Walk Theory, J. of App. Math. and Stoc. Anal. 11 (1) (1998) 9-28. 33

[43] C. Bardos, F. Golse, D. Levermore, Fluid Dynamics Limits of Discrete Velocity Kinetic Equations, in: N. Y. Springer-Verlag, Berlin (Ed.), Advances in Kinetic Theory and Continuum Mechanics, Gatignol and Soubbaramayer, Providence, Rhode Island, 57-71, 1991. 39 